HD-THEP-00-35

\title{
Spontaneously broken color
}

\author{
Christof Wetterich円 \\ Institut für Theoretische Physik \\ Universität Heidelberg \\ Philosophenweg 16, D-69120 Heidelberg
}

\begin{abstract}
The vacuum of QCD is characterized by the Higgs mechanism. Color is "spontaneously broken" by a quark-antiquark condensate in the octet representation. The massive gluons carry integer electric charges and are identified with the vector mesons. The fermionic excitations consist of the low mass baryon octet and a singlet. The interactions between these particles and the light pseudoscalar octet are largely determined by chiral symmetry and a nonlinear local symmetry. A consistent phenomenological picture of strong interactions at long distances arises from a simple effective action.
\end{abstract}

\footnotetext{
${ }^{1}$ e-mail: C.Wetterich@thphys.uni-heidelberg.de
} 


\section{Introduction}

An understanding of the properties of the vacuum is a central goal in the description of strong interactions by quantum chromodynamics (QCD). Recently, it has been proposed that the local color symmetry is broken spontaneously by a dynamical quark-antiquark condensate [1] [2]. The Higgs mechanism provides a mass for all gluons, thereby squeezing the gauge fields between color charges into flux tubes. The separation of two color charges leads therefore to an increasing potential until the string breaks due to particle production. This results in a simple picture of confinement. The Higgs mechanism also gives integer electric charge to all physical particles. Furthermore, the expectation value of the quark-antiquark color octet breaks the global chiral symmetry. In consequence the fermions become massive and the spectrum contains light pions and kaons as pseudo-Goldstone bosons. In the limit of equal masses for the three light quarks the global vector-like $\mathrm{SU}(3)$-symmetry of the "eightfold way" remains unbroken and can be used for a classification of the particle spectrum. The nine quarks transform as an octet and a singlet. We identify the octet with the low mass baryons this is quark-baryon duality. Similarly, the eight gluons carry the quantum numbers of the light vector mesons $\rho, K^{*}$ etc.. The identification of massive gluons with the vector mesons is called gluon-meson duality.

Strictly speaking, local symmetries cannot be broken spontaneously in the vacuum. This has led to the realisation that the confinement and the Higgs description are not necessarily associated to mutually exclusive phases. They may only be different facets of one and same physical state [3]. We stress that this observation is not only of formal importance. For example, the high temperature phase transition of electroweak interactions with a small Higgs scalar mass ends for a larger scalar mass in a critical endpoint. Beyond this endpoint the phase transition is replaced by an analytical crossover [ [ 1 ]. In this region - which is relevant for a realistic Higgs mass in the standard model - a Higgs and a confinement description can be used simultaneously. Our picture of the QCD vacuum ressembles in many aspects the "strongly coupled electroweak theory" at high temperature?

For strong interactions, the complementarity between the Higgs- and confinement description of the vacuum has been mainly discussed in toy models with additional fundamental colored scalar fields [6] In contrast, our approach concentrates on standard QCD with the gauge coupling and the current quark masses as the only free parameters. Similarly to chiral symmetry breaking the relevant dynamical condensate is provided by a quark-antiquark pair. The main difference to the usual treatment of chiral symmetry breaking

\footnotetext{
${ }^{2}$ Without a direct connection to the standard model the $S U(2)$-Yang-Mills theory with stong gauge coupling and fundamental scalar has been first simulated on the lattice in [5].

${ }^{3}$ See also ref. [7] for early discussions of strong interactions with additional fundamental colored scalar fields.
} 
is the assumption that both color singlet and octet composite fields acquire a vacuum expectation value.

We propose that besides the effective running of the gauge coupling the main ingredient of low-momentum QCD consists of effective scalar fields representing quark-antiquark bound states. Once these composite operators are treated on the same footing as the quark and gluon fields, the description of propagators and vertices in terms of an effective action becomes again very simple. The long-sought dual description of long-distance strong interactions can be realized by the addition of fields for composites. This is very similar to the asymptotically free nonlinear sigma model in two dimensions where the addition of the composite "radial excitation" provides for a simple dual description of the low momentum behavior [8]. We will call this particular version of duality where a simple effective action for the low momentum degrees of freedom can be achieved by the addition of fields for composite operators by the German wordf "Vervollständigung".

Spontaneous breaking of color has also been proposed [9] for situations with a very high baryon density, as perhaps in the interior of neutron stars. In this proposal a condensation of diquark operators is responsible for color superconductivity and spontaneous breaking of baryon number. In particular, the suggestion of color-flavor locking [10] offers analogies to our description of the vacuum, even though different physical situations are described (vacuum vs. high density state) and the pattern of spontaneous color-symmetry breaking is distinct (quark-antiquark vs. quark-quark condensate; conserved vs. broken baryon number). This analogy may be an important key for the understanding of possible phase transitions to a high density phase of QCD.

After spontaneous color symmetry breaking by an octet quark-antiquark condensate all quantum numbers of the excitations above the vacuum match with the observed spectrum of low mass particles. (These quantum numbers include baryon number, strangeness, isospin and spin as well as parity and C-parity.) We have therefore little doubt that such a description of the QCD vacuum is, in principle, possible. In the present paper we investigate the more specific hypothesis of "scalar Vervollständigung". This hypothesis states that the effective action becomes simple once one adds to the quark and gluon fields the scalar fields with the quantum numbers of quark-antiquark pairs. More precisely, we assume that in leading order only invariants with mass dimension smaller or equal to four have to be included, where the scalar fields are counted according to their canonical dimension. This "renormalizablef effective action" is assumed to be valid for low momenta. It exhibits a number of new couplings which describe the scalar potential and the Yukawa couplings between scalars and quarks. In principle, these couplings are calculable in QCD as a function of the gauge coupling or $\Lambda_{\mathrm{QCD}}$. In this work we

\footnotetext{
${ }^{4}$ To be translated roughly by "completion".

${ }^{5}$ Renormalizability is here no fundamental property since the validity of the effective action needs not to cover a large range of momenta.
} 
make no attempt of such a computation. (See ref. [2] for first steps in this direction.) We rather perform a phenomenological analysis treating the new couplings as free parameters. We concentrate on the limit of equal current quark masses. Besides the explicit chiral symmetry breaking by the current quark mass seven effective couplings are relevant for our discussion. Four of them appear only in the mass formulae for the light baryon octet and singlet and the $\eta^{\prime}$-meson. The masses and interactions (including interactions with baryons) of the pseudoscalar and vector mesons carrying isospin or strangeness are governed by only three effective parameters: the expectation values of the scalar octet $\left(\chi_{0}\right)$ and singlet $\left(\sigma_{0}\right)$ and the effective gauge coupling $(g)$. We fix $\chi_{0}, \sigma_{0}$ and $g$ by the observed values of the vector meson mass, the pseudoscalar decay constant and the electromagnetic decays of the vector mesons. "Predictions" for other quantities in this sector like the decay width for $\rho \rightarrow 2 \pi$ involve then no further free parameters. We will argue that the hypothesis of scalar Vervollständigung gives indeed a satisfactory description for long-distance strong interactions in leading order.

In sect. 2 we describe our setting in more detail and specify the transformation properties of the fields under the various symmetry transformations. In particular, the emergence of an integer electric charge for the excitations above the vacuum (physical particles) is discussed in sect. 3. Sect. 4 introduces a nonlinear description for the fields corresponding to physical particles. This makes the complementarity between the "Higgs picture" and the "confinement picture" manifest. In the nonlinear description the local color symmetry remains unbroken and all physical particles transform as color singlets. The remnant of the color symmetry for the interactions of the physical particles is a nonlinear local reparametrization symmetry. This is investigated in sect. 5. After a gauge fixing consistent with a simple realization of parity we arrive at the effective action for the nonlinear "physcial fields". In sect. 6 we turn to the issue of baryon number $B$. We address the puzzle how quarks with $B=1 / 3$ and baryons with $B=1$ can be described by the same field. In the Higgs language this issue is obscured by the fact that the gauge fixing is not compatible with the global transformation associated to $B$. A careful treatment reveals that baryons carry indeed three times the baryon number of quarks.

In sect. 7 we turn to the electromagnetic interactions for the nonlinear physical fields. They are largely governed by the nonlinear local reparametrization symmetry. We recover the well established concept of vector dominance as well as successful relations between hadronic and electromagnetic decays of the $\rho$-meson. Some shortcomings of the scalar Vervollständigung concerning the physics of the vector mesons are listed in sect. 8. Here we also propose an extension to "scalar-vector Vervollständigung" by adding fields for quarkantiquark bilinears in the vector and axial-vector channels. This completes the spectrum of light mesons by the ninth vector meson (the $S U(3)$-singlet) and the axial-vector mesons. In sect. 9 we discuss the effective interactions 
between the pseudoscalar mesons which follow from the hypothesis of scalar Vervollständigung. Their generic form is determined by chiral symmetry and we compute some of the effective couplings $L_{i}$ which appear in next-toleading order in chiral perturbation theory. The agreement with observation is very satisfactory. The weak interactions are introduced in sect. 10. There we show that the $\Delta I=1 / 2$ rule for the hadronic kaon decays arises naturally in our setting. In sect. 11 our discussion is extended to diquark fields which presumably play an important role for QCD in a medium with high baryon density. This opens new perspectives on the qualitative features of the QCD-phase diagram for large temperature and chemical potential. The short sect. 12 sketches the inclusion of the heavy quarks charm, beauty and top in our approach. We finally present a summary and conclusions in sect. 13.

\section{Quark-antiquark condensates}

\section{a) Effective action}

In this section we describe the effective action for long-distance strong interactions according to the hypothesis of scalar Vervollständigung. By definition the effective action generates the one-particle-irreducible (1PI) correlation functions and includes all quantum fluctuations. It therefore contains the direct information about the propagators and proper vertices.

In addition to the quark and gluon fields we consider scalar fields with the transformation properties of quark-antiquark pairs. With respect to the color and chiral flavor rotations $S U(3)_{C} \times S U(3)_{L} \times S U(3)_{R}$ the three light left-handed and right-handed quarks $\psi_{L}, \psi_{R}$ transform as $(3,3,1)$ and $(3,1,3)$, respectively. Quark-antiquark bilinears therefore contain a color singlet $\Phi(1, \overline{3}, 3)$ and a color octet $\chi(8, \overline{3}, 3)$

$$
\begin{aligned}
\gamma_{i j} & =\chi_{i j}+\frac{1}{\sqrt{3}} \phi \delta_{i j}, \\
\phi & =\frac{1}{\sqrt{3}} \gamma_{i i}, \quad \chi_{i i}=0
\end{aligned}
$$

Here we use a matrix notation for the flavor indices and write the color indices

$i, j$ explicitly, e.g. $\chi_{i j, a b} \equiv \chi_{i j}, \phi_{a b} \equiv \phi$. Then $\gamma_{i j}$ contains 81 complex scalar fields. Similarly, the quark fields are represented as three flavor vectors $\psi_{a i} \equiv$ $\psi_{i}, \bar{\psi}_{i a} \equiv \bar{\psi}_{i}$. The infinitesimal $S U(3)_{C} \times S U(3)_{L} \times S U(3)_{R}$ transformations are given by

$$
\begin{aligned}
& \left(\delta \psi_{L}\right)_{i}=i \psi_{L, j}\left(\Theta_{C}^{T}\right)_{j i}+i \Theta_{L} \psi_{L, i} \\
& \left(\delta \psi_{R}\right)_{i}=i \psi_{R, j}\left(\Theta_{C}^{T}\right)_{j i}+i \Theta_{R} \psi_{R, i}
\end{aligned}
$$

with

$$
\left(\Theta_{C}\right)_{i j}=\frac{1}{2} \theta_{C}^{z}(x)\left(\lambda_{z}\right)_{i j}, \Theta_{L, R}=\frac{1}{2} \theta_{L, R}^{z} \lambda_{z}
$$


Here $\lambda_{z}$ are the eight Gell-Mann matrices normalized according to $\operatorname{Tr}\left(\lambda_{y} \lambda_{z}\right)=$ $2 \delta_{y z}$ and $\Theta_{C}$ is a scalar in flavor space. Correspondingly, the scalar fields $\phi$ and $\gamma_{i j}$ transform as

$$
\begin{aligned}
& \delta \phi=i \Theta_{R} \phi-i \phi \Theta_{L}, \\
& \delta \chi_{i j}=i \Theta_{R} \chi_{i j}-i \chi_{i j} \Theta_{L}+i\left(\Theta_{C}\right)_{i k} \chi_{k j}-i \chi_{i k}\left(\Theta_{C}\right)_{k j}
\end{aligned}
$$

As usual we represent the eight $S U(3)_{C}$-gauge fields by

$$
\begin{aligned}
& A_{i j, \mu}=\frac{1}{2} A_{\mu}^{z}\left(\lambda_{z}\right)_{i j}, \\
& (\delta A)_{i j, \mu}=i\left(\Theta_{C}\right)_{i k} A_{k j, \mu}-i A_{i k, \mu}\left(\Theta_{C}\right)_{k j}+\frac{1}{g} \partial_{\mu}\left(\Theta_{C}\right)_{i j}
\end{aligned}
$$

We consider a very simple effective Lagrangian containing only terms with dimension up to four

$$
\begin{aligned}
\mathcal{L}= & i Z_{\psi} \bar{\psi}_{i} \gamma^{\mu} \partial_{\mu} \psi_{i}+g Z_{\psi} \bar{\psi}_{i} \gamma^{\mu} A_{i j, \mu} \psi_{j}+\frac{1}{2} G_{i j}^{\mu \nu} G_{j i, \mu \nu} \\
& +\operatorname{Tr}\left\{\left(D^{\mu} \gamma_{i j}\right)^{\dagger} D_{\mu} \gamma_{i j}\right\}+U(\gamma) \\
& +Z_{\psi} \bar{\psi}_{i}\left[\left(h \phi \delta_{i j}+\tilde{h} \chi_{i j}\right) \frac{1+\gamma_{5}}{2}-\left(h \phi^{\dagger} \delta_{i j}+\tilde{h} \chi_{j i}^{\dagger}\right) \frac{1-\gamma_{5}}{2}\right] \psi_{j}
\end{aligned}
$$

Here $G_{i j, \mu \nu}=\partial_{\mu} A_{i j, \nu}-\partial_{\nu} A_{i j, \mu}-i g A_{i k, \mu} A_{k j, \nu}+i g A_{i k, \nu} A_{k j, \mu}$ and the interaction between gluons and $\chi$ arise from the covariant derivative

$$
D_{\mu} \gamma_{i j}=\partial_{\mu} \gamma_{i j}-i g A_{i k, \mu} \gamma_{k j}+i g \gamma_{i k} A_{k j, \mu}
$$

In our notation the transposition acts only on flavor indices, e.g. $\left(\gamma_{i j}^{\dagger}\right)_{a b}=$ $\gamma_{i j, b a}^{*}$. The effective potential

$$
\begin{aligned}
& U(\gamma)=U_{0}(\chi, \phi)-\frac{1}{2} \nu\left(\operatorname{det} \phi+\operatorname{det} \phi^{\dagger}\right)-\frac{1}{2} \nu^{\prime}\left(E(\phi, \chi)+E^{*}(\phi, \chi)\right) \\
& E(\phi, \chi)=\frac{1}{6} \epsilon_{a_{1} a_{2} a_{3}} \epsilon_{b_{1} b_{2} b_{3}} \phi_{a_{1} b_{1}} \chi_{i j, a_{2} b_{2}} \chi_{j i, a_{3} b_{3}}
\end{aligned}
$$

conserves axial $U(1)$ symmetry except for the 't Hooft terms $12 \sim \nu, \nu^{\prime}$. For the purpose of this section the only information we need from $U_{0}$ concerns the expectation values $\sigma_{0}$ and $\chi_{0}$ for the singlet and octet in the limit of equal quark masses. The hypothesis of scalar Vervollständigung states that the leading behavior of low energy QCD can be directly extracted from the propagators and vertices of the effective action (2.6).

Finally, explicit chiral symmetry breaking is induced by a linear term 13

$$
\begin{aligned}
\mathcal{L}_{j} & =-\frac{1}{2} Z_{\phi}^{-1 / 2} \operatorname{Tr}\left(j^{\dagger} \phi+\phi^{\dagger} j\right) \\
j=j^{\dagger} & =a_{q} \bar{m}=a_{q} \operatorname{diag}\left(\bar{m}_{u}, \bar{m}_{d}, \bar{m}_{s}\right)
\end{aligned}
$$

\footnotetext{
${ }^{6}$ Four our conventions for fermions see 11 .
} 
with $\bar{m}_{q}$ the current quark masses normalized at some appropriate scale, say $\mu=2 \mathrm{GeV}$. We note that the quark wave function renormalization $Z_{\psi}$ can be absorbed by a rescaling of $\psi$. We keep it here in order to discuss later a possible simple bridge between our picture and the nonrelativistic quark model.

Besides the explicit chiral symmetry breaking in (2.9) the model contains the seven real parameters $g, h, \tilde{h}, \sigma_{0}, \chi_{0}, \nu$ and $\nu^{\prime}$. In fact, the effective action (2.6) is the most general' one which contains only "renormalizable" interactions and is consistent with $S U(3)_{C} \times S U(3)_{L} \times S U(3)_{R}$ symmetry, as well as with the discrete transformations parity and charge conjugation $(c$ is the charge conjugation matrix)

$$
\begin{aligned}
P: & \psi_{L} \rightarrow-\psi_{R}, \psi_{R} \rightarrow \psi_{L}, A_{i j, \mu} \rightarrow A_{i j, \mu}, \\
& \phi \rightarrow \phi^{\dagger}, \chi_{i j, a b} \rightarrow \chi_{j i, b a}^{*} \\
C: & \psi_{L} \rightarrow c \bar{\psi}_{R}, \psi_{R} \rightarrow-c \bar{\psi}_{L}, A_{i j, \mu} \rightarrow-A_{j i, \mu}, \\
& \phi \rightarrow \phi^{T}, \chi_{i j, a b} \rightarrow \chi_{j i, b a}
\end{aligned}
$$

We will show that the masses and interactions of the lightest octets of baryons as well as pseudoscalar and vector mesons are well described by the effective action (2.6), (2.9). A few shortcomings in the vector meson sector and a sketch of a possible extension are discussed in sect. 8 .

Actually, there is no reason why only interactions between scalar and pseudoscalar quark-antiquark bilinears should be present as in (2.6). Effective four-quark interactions (1PI) in the vector or axial-vector channel are certainly induced by fluctuations, and we will discuss them in appendix A. There is also no need that the effective action has to be of the "renormalizable form" (2.6). In fact, we have at present no strong argument why higher-order operators have to be small. In the spirit that a useful dual description should not be too complicated, we simply investigate in this paper to what extent the hypothesis of scalar Vervollständigung (2.6) is compatible with observation. If successful, the neglected subleading terms may be considered later for increased quantitative accuracy. Furthermore, we know that QCD contains higher resonances like the $\Delta$ or the axial-vector mesons. They are not described by the effective action (2.6). One may include them by the introduction of additional fields for bound states (see appendix A). This is, however, not the purpose of the present paper. We only mention here that "integrating out" the missing resonances with lowest mass presumably gives the leading contribution to the neglected higher-order operators. Those will typically contain "nonlocal behavior" in the momentum range characteristic for the resonances.

\footnotetext{
${ }^{7}$ The only exception is the omission of a possible $U(1)_{A}$-violating term cubic in $\chi$ which is not present in the 't Hooft interaction [12] and also not generated by one-loop fluctuations.
} 


\section{b) Connection to perturbative QCD}

In a renormalization group framework the parameters appearing in (2.6) can be considered as running coupling constants. For instance, we may associate $\Gamma_{k}=\int d^{4} x \mathcal{L}$ with the effective average action [14] which exhibits an infrared cutoff $k$. Then only quantum fluctuations with momenta larger than $k$ are included. The vacuum properties and the physical particle spectrum should be extracted for $k=0$. For short-distance scattering processes, however, one may account for the momentum dependence of the vertices by using for $k$ an appropriate momentum scale. Our description should coincide with perturbative QCD for large enough $k$.

Let us look first at the range of validity of perturbative QCD. For large $k$ (say $k \gtrsim 2 \mathrm{GeV}$ ) we expand $U_{0}=m_{\phi}^{2} \operatorname{Tr} \phi^{\dagger} \phi+m_{\chi}^{2} \operatorname{Tr} \chi_{i j}^{\dagger} \chi_{i j}+\ldots$ and assume that both $m_{\phi}^{2}$ and $m_{\chi}^{2}$ are large and positive. Then the additional scalar excitations will effectively decouple. They can be eliminated by solving the scalar field equations in terms of $\psi, \bar{\psi}$ and $A$ and reinserting this solution into the effective action. For $\psi=\bar{\psi}=0, A_{\mu}=0$ the expectation value $\langle\chi\rangle$ vanishes. On the other hand one finds $\langle\phi\rangle=\frac{1}{2} m_{\phi}^{-2} Z_{\Phi}^{-1 / 2} j$ and therefore effective quark masses

$$
\bar{m}_{q}=\frac{1}{2} h m_{\Phi}^{-2} Z_{\Phi}^{-1 / 2} j_{q} .
$$

This is the only relevant coupling induced by the additional degrees of freedom and exactly what is needed for perturbative QCD! We have explicitly checked that in leading order the running of the quark masses only arises from gluon diagrams. (The sources $j$ are constant and the effects of the running scalar wave function renormalization $Z_{\Phi}$ drop out.) Comparing with (2.9) we find for $k=\mu=2 \mathrm{GeV}$ the relation

$$
a_{q}(\mu)=2 m_{\phi}^{2}(\mu) Z_{\phi}^{1 / 2}(\mu) h^{-1}(\mu)
$$

For $A_{\mu}=0$ the exchange of $\phi$ and $\chi$ produces a correction $\Delta \mathcal{L}$

$$
\begin{aligned}
& \Delta \mathcal{L}=h^{2}\left(\bar{\psi}_{i a}\left(\frac{1-\gamma_{5}}{2}\right) \psi_{b i}\right)\left(m_{\phi}^{2}-\partial^{\mu} \partial_{\mu}\right)^{-1}\left(\bar{\psi}_{j b} \frac{1+\gamma_{5}}{2} \psi_{a j}\right) \\
& +\tilde{h}^{2}\left\{\left(\bar{\psi}_{a j} \frac{1-\gamma_{5}}{2} \psi_{b i}\right)\left(m_{\chi}^{2}-\partial^{\mu} \partial_{\mu}\right)^{-1}\left(\bar{\psi}_{b i} \frac{1+\gamma_{5}}{2} \psi_{a j}\right)\right. \\
& \left.-\frac{1}{3}\left(\bar{\psi}_{i a} \frac{1-\gamma_{5}}{2} \psi_{b i}\right)\left(m_{\chi}^{2}-\partial^{\mu} \partial_{\mu}\right)^{-1}\left(\bar{\psi}_{j b} \frac{1+\gamma_{5}}{2} \psi_{a j}\right)\right\}
\end{aligned}
$$

This corresponds to a one-particle irreducible effective four-quark interaction which can be computed perturbatively by evaluating the relevant box diagrams with two internal gluon and quark lines. One finds

$$
\frac{h^{2}(k)}{m_{\phi}^{2}(k)}=\frac{23 l_{3}^{4}}{144 \pi^{2}} \frac{g^{4}(k)}{k^{2}} \quad, \quad \frac{\tilde{h}(k)}{m_{\chi}^{2}(k)}=\frac{13 l_{3}^{4}}{384 \pi^{2}} \frac{g^{4}(k)}{k^{2}}
$$

with $l_{3}^{4}$ a constant of order one which depends on the precise choice of the infrared cutoff $k$. We emphasize the composite character of the scalar fields 
which implies that the $k$-dependence of $m_{\phi^{2}}$ or $h$ is essentially determined by the QCD-box diagrams if $k$ is large. Since the source $j$ is independent of the renormalization scale $\mu$, one infers from (2.9), (2.12) and (2.14) that the $k$-dependence of $Z_{\phi}$ obeys for the perturbative range

$$
Z_{\phi}^{1 / 2}(k) h(k) \sim \frac{g^{4}(k)}{k^{2} \bar{m}_{q}(k)}
$$

A small value of the scalar wave function renormalization $Z_{\phi}$ for large $k$ is consistent with a composite scalar field.

¿From the exchange of $\chi$ we also obtain for $A_{\mu} \neq 0$ higher-order gluon interactions by expanding $-\operatorname{Tr} \ln \left(m_{\chi}^{2}-D^{2}[A]\right)$ with $D^{2}$ the covariant Laplacian. All these corrections are nonrenormalizable interactions which are "irrelevant" by standard universality arguments. This means precisely that they are computable within the range of validity of perturbation theory. In fact, the scalar fields in $\Gamma_{k}$ may be viewed as a shorthand for the presence of these nonrenormalizable quark and gluon interactions in the effective action.

Due to the presence of quark fluctuations the mass terms $m_{\Phi}^{2}$ and $m_{\chi}^{2}$ decrease as $k$ is lowered. Perturbation theory typically breaks down once $m_{\Phi}^{2}$ or $m_{\chi}^{2}$ are of the same size as $k^{2}$. Furthermore, the relative importance of the anomaly terms increases. These cubic interactions tend to destabilize the minimum of the potential at $\phi=\chi=0$ (for $j=0$ ). It is our central postulate that in the nonperturbative region of small $k$ the minimum of the effective potential $U$ is located at nonzero expectation values 8 for both $\langle\phi\rangle$ and $\langle\chi\rangle$ even for $j=0$. A first dynamical analysis based on a mean field approximation indeed suggests [2] that this postulate is reasonable.

\section{c) Spontaneous symmetry breaking}

For small enough and equal quark masses the usual color singlet chiral quark condensate $<\bar{q} q>$ can be related to the expectaton value of $\phi$

$$
<\phi_{a b}>=\sigma_{0} \delta_{a b}
$$

by saturating the expectation value of the explicit chiral symmetry breaking term. From $\mathcal{L}_{j}=\sum_{q} \bar{m}_{q}(\mu)<\bar{q} q>(\mu)$ and (2.9), (2.11) one finds

$$
<\bar{q} q>(\mu)=-Z_{\phi}^{-1 / 2}(0) j_{q} \sigma_{0} \bar{m}_{q}(\mu)^{-1}=-\frac{2 m_{\phi}^{2}(\mu)}{h(\mu)}\left(\frac{Z_{\phi}(\mu)}{Z_{\phi}(0)}\right)^{1 / 2} \sigma_{0}
$$

The expectation value (2.16) breaks chiral symmetry. It preserves a vectorlike flavor symmetry $S U(3)_{\hat{V}}$ with transformations given by $\theta_{L}^{z}=\theta_{R}^{z}$ and color symmetry.

\footnotetext{
${ }^{8}$ The $S U(3)_{C}$ color breaking by nonzero $\langle\chi\rangle$ occurs only in a gauge-fixed version, whereas an explicitly gauge-invariant formulation is used below.
} 
We suggest that the essential features of confinement are described by nonzero $\langle\chi\rangle$. For equal quark masses we propose

$$
<\chi_{i j, a b}>=\frac{1}{\sqrt{24}} \chi_{0} \lambda_{j i}^{z} \lambda_{a b}^{z}=\frac{1}{\sqrt{6}} \chi_{0}\left(\delta_{i a} \delta_{j b}-\frac{1}{3} \delta_{i j} \delta_{a b}\right)
$$

with real $\chi_{0}>0$. This expectation value is invariant under vectorlike $S U(3)_{V}$ transformations by which identical left and right flavor rotations and transposed color rotations are performed with opposite angles. More explicitly, they are given by $\theta_{L}^{z}=\theta_{R}^{z}=\epsilon^{(z)} \theta_{C}^{z} \equiv \theta_{V}^{z}$ with $\epsilon^{(z)}=-1$ for $z=1,3,4,6,8$ and $\epsilon^{(z)}=1$ for $z=2,5,7$. The $S U(3)_{V}$-transformation properties of the various fields follow by inserting in eqs. (2.2), (2.4) and (2.5) $\Theta_{L}=\Theta_{R}=-\left(\Theta_{C}\right)^{T} \equiv \Theta_{V}$

$$
\begin{aligned}
\delta_{V} \psi_{a i}= & i\left(\Theta_{V}\right)_{a b} \psi_{b i}-i \psi_{a j}\left(\Theta_{V}\right)_{j i} \\
\delta_{V} A_{i j, \mu}^{T}= & i\left(\Theta_{V}\right)_{i k} A_{k j, \mu}^{T}-i A_{i k, \mu}^{T}\left(\Theta_{V}\right)_{k j} \\
\delta_{V} \Phi_{a b}= & i\left(\Theta_{V}\right)_{a c} \phi_{c b}-i \Phi_{a c}\left(\Theta_{V}\right)_{c b} \\
\delta_{V} \chi_{i j, a b}= & i\left(\Theta_{V}\right)_{a c} \chi_{i j, c b}-i \chi_{i j, a c}\left(\Theta_{V}\right)_{c b} \\
& -i\left(\Theta_{V}\right)_{k i} \chi_{k j, a b}+i \chi_{i k, a b}\left(\Theta_{V}\right)_{j k}
\end{aligned}
$$

The invariance of the expectation value $\left.\delta_{V}<\chi_{i j, a b}\right\rangle=0$ is easily checked. With respect to the transposed color rotations the quarks behave as antitriplets. In consequence, under the combined transformation they transform as an octet plus a singlet, with all quantum numbers identical to the baryon octet/singlet. This is most obvious if we also use a matrix notation for the fermions $\psi \equiv \psi_{a i}$ such that $\delta_{V} \psi=i\left[\Theta_{V}, \psi\right]$. In particular, the electric charges, as given by the generator $Q=\frac{1}{2} \lambda_{3}+\frac{1}{2 \sqrt{3}} \lambda_{8}$ of $S U(3)_{V}$, are integer. We therefore identify the quark field with the lowest baryon octet and singlet - this is quark-baryon duality. Similarly, the gluons transform as an octet of vector-mesons, again with the standard charges for the $\rho, K^{*}$ and $\omega / \phi$ mesons. We therefore describe these vector mesons by the gluon field - this is gluon-meson duality.

Due to the Higgs mechanism all gluons acquire a mass. For equal quark masses conserved $S U(3)_{V}$ symmetry implies that all masses are equal. They should be identified with the average mass of the vector meson multiplet $\bar{M}_{\rho}^{2}=\frac{2}{3} M_{K^{*}}^{2}+\frac{1}{3} M_{\rho}^{2}=(850 \mathrm{MeV})^{2}$. One finds

$$
\bar{M}_{\rho}=g \chi_{0}=850 \mathrm{MeV}
$$

The breaking of chiral symmetry by $\sigma_{0}$ and $\chi_{0}$ also induces masses for the baryon octet and singlet. For $\chi_{0} \neq 0$ the singlet mass is larger than the octet mass (see below).

We note that gluon-meson duality is a necessary consequence of the Higgs picture of QCD. It persists if additional vector-meson fields are introduced as color singlet quark-antiquark composite fields. There will simply be a mixing 
between the "gluons" and the "composite vector fields" (see sect. 8). On the other hand, quark baryon duality strongly depends on the proper assignment of the baryon number. We will discuss this issue in detail in sect. 6 .

\section{$3 \quad$ Integer electric charges}

In order to get familiar with the Higgs picture of QCD, it seems useful to understand in more detail the consequences of spontaneous color symmetry breaking for the electromagnetic interactions of hadrons. We start by adding to the effective Lagrangian (2.6) a coupling to a $U(1)$-gauge field $\tilde{B}_{\mu}$ by making derivatives covariant

$$
\begin{aligned}
D_{\mu} \psi & =\left(\partial_{\mu}-i \tilde{e} \tilde{Q} \tilde{B}_{\mu}\right) \psi \\
D_{\mu} \gamma_{i j} & =\partial_{\mu} \gamma_{i j}-i \tilde{e}\left[\tilde{Q}, \gamma_{i j}\right] \tilde{B}_{\mu}-\ldots
\end{aligned}
$$

with $\tilde{Q}=\frac{1}{2} \lambda_{3}+\frac{1}{2 \sqrt{3}} \lambda_{8}=\operatorname{diag}\left(\frac{2}{3},-\frac{1}{3},-\frac{1}{3}\right)$ acting on the flavor indices. Furthermore we supplement the Maxwell term

$$
\mathcal{L}_{\tilde{B}}=\frac{1}{4} \tilde{B}^{\mu \nu} \tilde{B}_{\mu \nu}, \tilde{B}_{\mu \nu}=\partial_{\mu} \tilde{B}_{\nu}-\partial_{\nu} \tilde{B}_{\mu}
$$

Whereas the quarks carry fractional $\tilde{Q}$, the abelian charges of the scalars are integer. In particular, the expectation value $\langle\phi\rangle$ is neutral, $[\tilde{Q},\langle\phi\rangle]=0$, whereas some components of $\left\langle\chi_{i j, a b}>\right.$ carry charge, namely for $k=2,3$

$$
\begin{aligned}
& {\left[\tilde{Q},<\chi_{1 k, 1 k}>\right]=<\chi_{1 k, 1 k}>} \\
& {\left[\tilde{Q},<\chi_{k 1, k 1}>\right]=-<\chi_{k 1, k 1}>}
\end{aligned}
$$

The expectation value (2.18) therefore also breaks the local $U(1)$ symmetry associated with $\tilde{Q}$. The abelian color charge $\left(Q_{C}\right)_{i j}=\frac{1}{2}\left(\lambda_{3}\right)_{i j}+\frac{1}{2 \sqrt{3}}\left(\lambda_{8}\right)_{i j}$ of these fields is, however, equal to $\tilde{Q}$. In consequence, a local abelian symmetry with generator $\tilde{Q}-Q_{C}$ remains unbroken

$$
\tilde{Q}_{a c}<\chi_{i j, c b}>-<\chi_{i j, a c}>\tilde{Q}_{c b}-\left(Q_{C}\right)_{i l}<\chi_{l j, a b}>+<\chi_{i l, a b}>\left(Q_{C}\right)_{l j}=0
$$

The corresponding gauge field corresponds to the photon.

The situation encountered here is completely analogous to the Higgs mechanism in electroweak symmetry breaking. The mixing between the hypercharge boson and the $W_{3}$ boson in the electroweak theory appears here as a mixing between $\tilde{B}_{\mu}$ and a particular gluon field $\tilde{G}_{\mu}$ which corresponds to $A_{i j, \mu}=\frac{\sqrt{3}}{2}\left(Q_{C}\right)_{i j} \tilde{G}_{\mu}$. Let us restrict the discussion to the gauge bosons $\tilde{B}_{\mu}$ and $\tilde{G}_{\mu}$. Then the covariant derivative for fields with a fixed value of $\tilde{Q}$ and $Q_{C}$ is given by

$$
D_{\mu}=\partial_{\mu}-i \tilde{e} \tilde{B}_{\mu} \tilde{Q}-i \tilde{g} \tilde{G}_{\mu} Q_{C}
$$


with $\tilde{g}=\frac{\sqrt{3}}{2} g$. Due to the "charged" expectation values (3.3) a linear combination of $\tilde{B}_{\mu}$ and $\tilde{G}_{\mu}$ gets massive, as can be seen from the quadratic Lagrangian

$$
\mathcal{L}_{e m}^{(2)}=\frac{1}{4} \tilde{B}^{\mu \nu} \tilde{B}_{\mu \nu}+\frac{1}{4} \tilde{G}^{\mu \nu} \tilde{G}_{\mu \nu}+\frac{2}{3} \chi_{0}^{2}\left(\tilde{g} \tilde{G}^{\mu}+\tilde{e} \tilde{B}^{\mu}\right)\left(\tilde{g} \tilde{G}_{\mu}+\tilde{e} \tilde{B}_{\mu}\right)
$$

The massive neutral vector meson $R_{\mu}$ and the massless photon $B_{\mu}$ are related to $\tilde{G}_{\mu}$ and $\tilde{B}_{\mu}$ by a mixing angle

$$
\begin{aligned}
R_{\mu} & =\cos \theta_{e m} \tilde{G}_{\mu}+\sin \theta_{e m} \tilde{B}_{\mu} \\
B_{\mu} & =\cos \theta_{e m} \tilde{B}_{\mu}-\sin \theta_{e m} \tilde{G}_{\mu} \\
t g \theta_{e m} & =\frac{\tilde{e}}{\tilde{g}}
\end{aligned}
$$

and we note that the mass of the neutral vector meson is somewhat enhanced by the mixing

$$
M_{V_{0}}=g \chi_{0} / \cos \theta_{e m}
$$

The mixing is, however, tiny for the large value $\bar{\alpha}_{s}=g^{2} / 4 \pi \approx 3$ that we will find below. In terms of the mass eigenstates the covariant derivative (3.5) reads now

$$
D_{\mu}=\partial_{\mu}-i e Q B_{\mu}-i \tilde{g} \cos \theta_{e m}\left(Q_{C}+t g^{2} \theta_{e m} \tilde{Q}\right) R_{\mu}
$$

and we observe the universal electromagnetic coupling

$$
e=\tilde{e} \cos \theta_{e m}
$$

of all particles with electric charge $Q=\tilde{Q}-Q_{C}$. This coupling is exactly the same for the colored quarks and the colorless leptons as it should be for the neutrality of atoms. For an illustration we show the charges $Q_{C}, \tilde{Q}$ and $Q$ for the nine light quarks in table 1 .

We note the difference between the coupling $\tilde{e}$ which would be computed in a grand unified theory and the true electromagnetic coupling $e$. They are related by

$$
\frac{1}{\tilde{e}^{2}}=\frac{1}{e^{2}}-\frac{4}{3 g^{2}}
$$

For $g=6$ the relative correction is only of the order of $10^{-3}$.

\section{Nonlinear meson interactions}

The interactions of the light mesons are most easily described in an equivalent gauge-invariant picture, using nonlinear fields?" For the "Goldstone

\footnotetext{
${ }^{9}$ On the level of the effective action the quantum fluctuations are already included. Therefore Jacobians for nonlinear field transformations play no role. All "coordinate choices" in the space of fields are equivalent.
} 


\begin{tabular}{c|ccc|c|} 
& $\tilde{Q}$ & $Q_{c}$ & $Q$ & \\
\hline$u_{1}$ & $2 / 3$ & $2 / 3$ & 0 & $\Sigma^{0}, \Lambda^{0}, S^{0}$ \\
$u_{2}$ & $2 / 3$ & $-1 / 3$ & 1 & $\Sigma^{+}$ \\
$u_{3}$ & $2 / 3$ & $-1 / 3$ & 1 & $p$ \\
\hline$d_{1}$ & $-1 / 3$ & $2 / 3$ & -1 & $\Sigma^{-}$ \\
$d_{2}$ & $-1 / 3$ & $-1 / 3$ & 0 & $\Sigma^{0}, \Lambda^{0}, S^{0}$ \\
$d_{3}$ & $-1 / 3$ & $-1 / 3$ & 0 & $n$ \\
\hline$s_{1}$ & $-1 / 3$ & $2 / 3$ & -1 & $\Xi^{-}$ \\
$s_{2}$ & $-1 / 3$ & $-1 / 3$ & 0 & $\Xi^{0}$ \\
$s_{3}$ & $-1 / 3$ & $-1 / 3$ & 0 & $\Lambda^{0}, S^{0}$
\end{tabular}

Table 1: Abelian charges of quarks and association with baryons. The baryon singlet is denoted by $S^{0}$.

directions" we introduce unitary matrices $W_{L}, W_{R}, v$ and define

$$
\begin{aligned}
\psi_{L} & =Z_{\psi}^{-1 / 2} W_{L} N_{L} v, \psi_{R}=Z_{\psi}^{-1 / 2} W_{R} N_{R} v \\
\bar{\psi}_{L} & =Z_{\psi}^{-1 / 2} v^{\dagger} \bar{N}_{L} W_{L}^{\dagger}, \bar{\psi}_{R}=Z_{\psi}^{-1 / 2} v^{\dagger} \bar{N}_{R} W_{R}^{\dagger} \\
A_{\mu} & =-v^{T} V_{\mu}^{T} v^{*}-\frac{i}{g} \partial_{\mu} v^{T} v^{*}, U=W_{R} W_{L}^{\dagger}, \\
\phi & =W_{R} S W_{L}^{\dagger}, \chi_{i j, a b}=\left(W_{R}\right)_{a c} v_{i k}^{T} X_{k l, c d} v_{l j}^{*}\left(W_{L}^{\dagger}\right)_{d b}
\end{aligned}
$$

Here we have again extended the matrix notation to the color indices with the quark/baryon nonet represented by a complex $3 \times 3$ matrix $\psi \equiv \psi_{a i}, \bar{\psi} \equiv \bar{\psi}_{i a}$. The decomposition of $\phi$ is such that $S$ is a hermitean matrix. The restrictions on $X$, which are necessary to avoid double counting, play no role in the present work. Besides $v$ all nonlinear fields are color singlets. The field $v^{\dagger}$ transforms as a color anti-triplet similar to a quark-quark pair or diquark. In consequence, the baryons $N \sim \psi v^{\dagger}$ transform as a color singlet. More precisely, the $S U(3)_{C} \times S U(3)_{L} \times S U(3)_{R}$ transformations of the nonlinear fields are given by

$$
\begin{aligned}
& \delta W_{L}=i \Theta_{L} W_{L}-i W_{L} \Theta_{P}, \delta W_{R}=i \Theta_{R} W_{R}-i W_{R} \Theta_{P} \\
& \delta v=i \Theta_{P} v+i v \Theta_{C}^{T}, \delta U=i \Theta_{R} U-i U \Theta_{L} \\
& \delta N_{L}=i\left[\Theta_{P}, N_{L}\right], \delta N_{R}=i\left[\Theta_{P}, N_{R}\right], \delta V_{\mu}=i\left[\Theta_{P}, V_{\mu}\right]+\frac{1}{g} \partial_{\mu} \Theta_{P} \\
& \delta S=i\left[\Theta_{P}, S\right],(\delta X)_{i j, a b}=i\left(\Theta_{P}\right)_{a c} X_{i j, c b}-i X_{i j, a c}\left(\Theta_{P}\right)_{c b} \\
&-i\left(\Theta_{P}^{T}\right)_{i k} X_{k j, a b}+i X_{i k, a b}\left(\Theta_{P}^{T}\right)_{k j}
\end{aligned}
$$

We observe the appearance of a new local symmetry $U(3)_{P}=S U(3)_{P} \times$ $U(1)_{P}$ under which the nucleons $N$ and the vector meson fields $V_{\mu}$ transform 
as octets and singlets

$$
\Theta_{P}=\frac{1}{2}\left(\theta_{P}^{z}(x) \lambda_{z}+\theta_{P}^{0}(x) \lambda_{0}, \lambda_{0} \equiv \frac{2}{\sqrt{6}} .\right.
$$

This symmetry acts only on the nonlinear fields whereas $\psi, A_{\mu}, \phi$ and $\chi$ are invariant. It reflects the possibility of local reparametrizations of the nonlinear fields. It will play the role of the hidden gauge symmetry underlying vector dominance. Furthermore, there is an additional local abelian symmetry $U(1)_{N}$ (not shown in eq. (4.2)

$$
v \rightarrow e^{-i \alpha(x)} v, N \rightarrow e^{i \alpha(x)} N, V_{\mu} \rightarrow V_{\mu}-\frac{1}{g} \partial_{\mu} \alpha(x)
$$

and a global symmetry (with charge denoted by $B_{V}$ )

$$
\psi \rightarrow e^{i \frac{\gamma}{3}} \psi, W_{L, R} \rightarrow e^{i \frac{\gamma}{3}} W_{L, R}
$$

For the present investigation we omit the scalar excitations except for the "Goldstone directions" contained in $W_{L}, W_{R}, v$. We can therefore replace $X_{k l, a b}$ by the expectation value (2.18) and use $\langle S\rangle=\sigma_{0}$ such that

$$
\Phi=\sigma_{0} U \quad, \quad \chi_{i j, a b}=\frac{1}{\sqrt{6}} \chi_{0}\left\{\left(W_{R} v\right)_{a i}\left(v^{\dagger} W_{L}^{\dagger}\right)_{j b}-\frac{1}{3} U_{a b} \delta_{i j}\right\}
$$

In terms of the nonlinear field coordinates the Lagrangian (2.6) reads

$$
\begin{aligned}
\mathcal{L}= & \operatorname{Tr}\left\{i \bar{N} \gamma^{\mu}\left(\partial_{\mu}+i v_{\mu}-i \gamma^{5} a_{\mu}\right) N-g \bar{N} \gamma^{\mu} N V_{\mu}\right\} \\
& +\frac{1}{2} \operatorname{Tr}\left\{V^{\mu \nu} V_{\mu \nu}\right\}+g^{2} \chi_{0}^{2} \operatorname{Tr}\left\{\tilde{V}^{\mu} \tilde{V}_{\mu}\right\}+\left(\frac{2}{9} \nu^{\prime} \chi_{0}^{2} \sigma_{0}-\nu \sigma_{0}^{3}\right) \cos \theta \\
& +\left(h \sigma_{0}-\frac{\tilde{h}}{3 \sqrt{6}} \chi_{0}\right) \operatorname{Tr}\left\{\bar{N} \gamma_{5} N\right\}+\frac{\tilde{h}}{\sqrt{6}} \operatorname{Tr} \bar{N} \gamma^{5} \operatorname{Tr} N \\
& +\left(\sigma_{0}^{2}+\frac{7}{36} \chi_{0}^{2}\right) \operatorname{Tr}\left\{\partial^{\mu} U^{\dagger} \partial_{\mu} U\right\}+\frac{1}{12} \chi_{0}^{2} \partial^{\mu} \theta \partial_{\mu} \theta \\
& +\chi_{0}^{2} \operatorname{Tr}\left\{\tilde{v}^{\mu} \tilde{v}_{\mu}\right\}+2 g \chi_{0}^{2} \operatorname{Tr}\left\{\tilde{V}^{\mu} \tilde{v}_{\mu}\right\}
\end{aligned}
$$

with

$$
\begin{aligned}
& v_{\mu}=-\frac{i}{2}\left(W_{L}^{\dagger} \partial_{\mu} W_{L}+W_{R}^{\dagger} \partial_{\mu} W_{R}\right), \\
& a_{\mu}=\frac{i}{2}\left(W_{L}^{\dagger} \partial_{\mu} W_{L}-W_{R}^{\dagger} \partial_{\mu} W_{R}\right)=-\frac{i}{2} W_{R}^{\dagger} \partial_{\mu} U W_{L}, \\
& \operatorname{Tr} v_{\mu}=-\frac{i}{2} \partial_{\mu}\left(\ln \operatorname{det} W_{L}+\ln \operatorname{det} W_{R}\right), \\
& \operatorname{Tr} a_{\mu}=-\frac{i}{2} \operatorname{Tr} U^{\dagger} \partial_{\mu} U=-\frac{1}{2} \partial_{\mu} \theta, \\
& V_{\mu \nu}=\partial_{\mu} V_{\nu}-\partial_{\nu} V_{\mu}-i g\left[V_{\mu}, V_{\nu}\right]=\partial_{\mu} \tilde{V}_{\nu}-\partial_{\nu} \tilde{V}_{\mu}-i g\left[\tilde{V}_{\mu}, \tilde{V}_{\nu}\right], \\
& \tilde{V}_{\mu}=V_{\mu}-\frac{1}{3} \operatorname{Tr} V_{\mu}, \tilde{v}_{\mu}=v_{\mu}-\frac{1}{3} \operatorname{Tr} v_{\mu}
\end{aligned}
$$


As it should be, $\mathcal{L}$ does not depend on $v$ and is therefore invariant under $S U(3)_{C}$ transformations. For a check of local $U(3)_{P}$ invariance we note

$$
\delta v_{\mu}=i\left[\Theta_{P}, v_{\mu}\right]-\partial_{\mu} \Theta_{P}, \delta a_{\mu}=i\left[\Theta_{P}, a_{\mu}\right]
$$

such that $v_{\mu}$ transforms the same as $-g V_{\mu}$ and $v_{\mu}+g V_{\mu}$ transforms homogeneously. One observes that the mass term for $\tilde{V}_{\mu}$ appears in the $U(3)_{P^{-}}$ invariant combination $\chi_{0}^{2} \operatorname{Tr}\left\{\left(\tilde{v}^{\mu}+g \tilde{V}^{\mu}\right)\left(\tilde{v}_{\mu}+g \tilde{V}_{\mu}\right)\right\}$. The nonlinear field $U$ only appears in derivative terms except for the phase $\theta$ associated to the $\eta^{\prime}$-meson. We associate $U$ in the standard way with the pseudoscalar octet of Goldstone bosons $\Pi^{z}$

$$
U=\exp \left(-\frac{i}{3} \theta\right) \exp \left(i \frac{\Pi^{z} \lambda_{z}}{f}\right)=\exp \left(-\frac{i}{3} \theta\right) \tilde{U}
$$

where the decay constant $f$ will be specified below.

¿From eq. (4.7) one can directly read the average mass of the baryon octet and singlett

$$
M_{8}=h \sigma_{0}-\frac{\tilde{h}}{3 \sqrt{6}} \chi_{0}=1.15 \mathrm{GeV}, M_{1}=h \sigma_{0}+\frac{8}{3} \frac{\tilde{h}}{\sqrt{6}} \chi_{0}=1.6(1.8) \mathrm{GeV}
$$

The singlet-octet mass splitting is proportional to the octet condensate $\chi_{0}$

$$
M_{1}-M_{8}=\frac{3}{\sqrt{6}} \tilde{h} \chi_{0}=450(650) \mathrm{MeV}
$$

With eq. (2.20) this determines the ratio

$$
\frac{\tilde{h}}{g}=0.43(0.62)
$$

Similarly, one finds

$$
h \sigma_{0}=\frac{1}{9}\left(8 M_{8}+M_{1}\right)=1.2(1.22) \mathrm{GeV}
$$

\section{Local reparametrization symmetry}

Before extracting the couplings between the physical mesons and the baryons we have to understand the role of the local reparametrization transformations $U(3)_{P}=S U(3)_{P} \times U(1)_{P}$. First of all, we note that $\operatorname{Tr} v_{\mu}$ can be eliminated by $U(1)_{P}$-gauge transformations and is therefore a pure gauge degree of freedom. Similarly, $\operatorname{Tr} V_{\mu}=-\frac{i}{g} \partial_{\mu} \ln$ det $v$ is the gauge degree of

\footnotetext{
${ }^{10}$ The lowest state with the appropriate quantum numbers for the singlet is $\Lambda(1600)$. Since we expect a very broad decay width of the singlet this assignment is very uncertain and we add in brackets a somewhat higher value.
} 
freedom corresponding to $U(1)_{N}$ transformations. This explains why $\operatorname{Tr} v_{\mu}$ and $\operatorname{Tr} V_{\mu}$ only appear in the term bilinear in the nucleon fields. The vector fields $\tilde{V}_{\mu}$ are the gauge bosons of $S U(3)_{P}$. The nucleons transform as an octet and a singlet under $S U(3)_{P}$ and the same holds for the bilinear $a_{\mu}$. The fields contained in $W_{L}, W_{R}$ are antitriplets and $U$ is a singlet.

In a gauge-fixed version the vacuum corresponds to $W_{L}=W_{R}=v=$ 1. The remaining global symmetry ${ }^{11} S U(3)_{\tilde{V}}$ is a linear combination of $S U(3)_{L}, S U(3)_{R}, S U(3)_{C}$ and $S U(3)_{P}$ given by transformations obeying

$$
\Theta_{\tilde{V}}=\Theta_{L}=\Theta_{R}=-\left(\Theta_{C}\right)^{T}=\Theta_{V}=\Theta_{P}
$$

With respect to $S U(3)_{\tilde{V}}$ the nonlinear fields and bilinears $W_{L}, W_{R}, v$, $U, N, V_{\mu}$, $a_{\mu}, v_{\mu}$ transform all as octets or singlets. On the level of the nonlinear fields the global $S U(3)_{\tilde{V}}$ symmetry plays the same role as $S U(3)_{V}$ for the linear fields. One may identify $S U(3)_{V}$ and $S U(3)_{\tilde{V}}$ by specifying the $S U(3)_{V^{-}}$ transformations of the nonlinear fields by the choice $\Theta_{P}=\Theta_{V}$.

For a discussion of the mesons we have to eliminate the redundance of the local $U(3)_{P}$-transformations. We first argue that the appropriate choice of a gauge fixing requires some care. As an example, one may explore the possible gauge fixing $W_{R}=v=1$. Then $U=W_{L}^{\dagger}, v_{\mu}=-a_{\mu}=-\frac{i}{2} U \partial_{\mu} U^{\dagger}=\frac{i}{2} \partial_{\mu} U U^{\dagger}$ would yield

$$
\begin{gathered}
\frac{7}{36} \operatorname{Tr}\left\{\partial^{\mu} U^{\dagger} \partial_{\mu} U\right\}+\frac{1}{12} \partial^{\mu} \theta \partial_{\mu} \theta+\operatorname{Tr}\left\{\tilde{v}^{\mu} \tilde{v}_{\mu}\right\}=\frac{4}{9} \operatorname{Tr}\left\{\partial^{\mu} U^{\dagger} \partial_{\mu} U\right\} \\
2 \operatorname{Tr}\left\{\tilde{V}^{\mu} \tilde{v}_{\mu}\right\}=-i \operatorname{Tr}\left\{U \partial_{\mu} U^{\dagger} V^{\mu}\right\}
\end{gathered}
$$

This is the language used $\mathrm{H}^{2}$ in ref [1]. If one would associate the physical pions with $\Pi_{z}$, one would obtain for the decay constant $f=2 \sqrt{\sigma_{0}^{2}+4 \chi_{0}^{2} / 9}$. We note, however, that in this language the vector bilinear $v_{\mu}$ has a contribution linear in the pion fields, $v_{\mu}=-(1 / 2 f) \lambda_{z} \partial_{\mu} \Pi^{z}+\partial_{\mu} \theta / 6+\ldots$ The term (5.3) leads therefore to a field mixing in quadratic oder $\sim \partial^{\mu} \Pi^{z} V_{\mu}^{z}$. We conclude that the propagators for $\Pi^{z}$ and $V_{\mu}^{z}$ are not in standard diagonal form in this gauge. The corresponding fields can therefore not be associated with physical particles in this language. This point was overlooked in ref. [1].

On the other hand, the gauge choice

$$
W_{L}^{\dagger}=W_{R}=\xi, v=1
$$

implies

$$
U=\xi^{2}, v_{\mu}=-\frac{i}{2}\left(\xi^{\dagger} \partial_{\mu} \xi+\xi \partial_{\mu} \xi^{\dagger}\right)
$$

\footnotetext{
${ }^{11}$ The abelian part of the symmetry will be discussed in detail in sect. 6 .

${ }^{12}$ The field $V_{\mu}$ in ref. [1] corresponds to $-V_{\mu}^{T}$ in the present notation.
} 


$$
\begin{aligned}
& a_{\mu}=-\frac{i}{2}\left(\xi^{\dagger} \partial_{\mu} \xi-\xi \partial_{\mu} \xi^{\dagger}\right)=-\frac{i}{2} \xi^{\dagger} \partial_{\mu} U \xi^{\dagger} \\
& \operatorname{Tr} v_{\mu}=0, \quad \operatorname{Tr} V_{\mu}=0 \\
& \phi=\sigma_{0} U, \xi_{i j, a b}=\frac{1}{\sqrt{6}} \chi_{0}\left(\xi_{a i} \xi_{j b}-\frac{1}{3} U_{a b} \delta_{i j}\right) \\
& \left(D_{\mu} \chi\right)_{i j, a b}=\frac{1}{\sqrt{6}} \chi_{0} . \\
& \quad\left[\partial_{\mu}\left(\xi_{a i} \xi_{j b}-\frac{1}{3} U_{a b} \delta_{i j}\right)+i g\left(\left(\xi \tilde{V}_{\mu}\right)_{a i} \xi_{j b}-\xi_{a i}\left(\tilde{V}_{\mu} \xi\right)_{j b}\right)\right]
\end{aligned}
$$

This gauge is singled out by the fact that $v_{\mu}$ contains no term linear in $\Pi^{z}$ or $\theta$

$$
v_{\mu}=\tilde{v}_{\mu}=-\frac{i}{2 f^{2}}\left[\Pi, \partial_{\mu} \Pi\right]=-\frac{i}{8 f^{2}}\left[\lambda_{y}, \lambda_{z}\right] \Pi^{y} \partial_{\mu} \Pi^{z}+\ldots
$$

where we have chosen the parametrization

$$
\begin{aligned}
& \xi=\tilde{\xi} \exp \left(-\frac{i}{6} \theta\right), \operatorname{det} \tilde{\xi}=1 \\
& \tilde{\xi}=\exp \left(\frac{i}{2 f} \Pi^{z} \lambda_{z}\right)=\exp \left(\frac{i}{f} \Pi\right)
\end{aligned}
$$

As for any gauge where $v_{\mu}$ contains no term linear in $\Pi$ or $\theta$, the term $\sim \operatorname{Tr} \tilde{v}^{\mu} \tilde{V}_{\mu}$ contains only interactions and does not affect the propagators. We can therefore associate $\Pi^{z}$ with the physical pions. Also the term $\operatorname{Tr} \tilde{v}^{\mu} \tilde{v}_{\mu}$ involves at least four meson fields and is irrelevant for the propagator of the pseudoscalar mesons.

The bilinear $a_{\mu}$ reads in the gauge (5.4)

$$
a_{\mu}=\frac{1}{2 f} \lambda_{z} \partial_{\mu} \Pi^{z}-\frac{1}{6} \partial_{\mu} \theta+O\left(\Pi^{3}, . .\right)
$$

The effective action (4.7) contains therefore a cubic coupling between two baryons and a pion. As it should be for pseudo-Goldstone bosons, this is a derivative coupling. Actually, the advantage of the gauge (5.4) can also be understood from the point of view of the discrete symmetries $P$ and $C$. A simple realisation of the discrete transformations on the level of nonlinear fields is $[3$

$$
\begin{aligned}
P: & N_{L} \rightarrow-N_{R}, N_{R} \rightarrow N_{L}, \\
& W_{L} \rightarrow W_{R}, W_{R} \rightarrow W_{L}, v \rightarrow v, U \rightarrow U^{\dagger}, \xi \rightarrow \xi^{\dagger} \\
& v_{\mu} \rightarrow v_{\mu}, a_{\mu} \rightarrow-a_{\mu}, V_{\mu} \rightarrow V_{\mu} \\
& \\
C: \quad & N_{L} \rightarrow c \bar{N}_{R}^{T}, N_{R} \rightarrow-c \bar{N}_{L}^{T}, \\
& W_{L} \rightarrow W_{R}^{*}, W_{R} \rightarrow W_{L}^{*}, v \rightarrow v^{*}, U \rightarrow U^{T}, \xi \rightarrow \xi^{T} \\
& v_{\mu} \rightarrow-v_{\mu}^{T}, a_{\mu} \rightarrow a_{\mu}^{T}, V_{\mu} \rightarrow-V_{\mu}^{T}
\end{aligned}
$$

\footnotetext{
${ }^{13}$ Note that in our matrix notation $C$ acts as $\psi_{L} \rightarrow c \bar{\psi}_{R}^{T}, \psi_{R} \rightarrow-c \bar{\psi}_{L}^{T}$.
} 
The gauge condition (5.4) can also be written as $W_{L} W_{R}=W_{R} W_{L}=1$ and is manifestly invariant under $P$ and $C$. The coupling $\operatorname{Tr} \bar{N} \gamma^{\mu} \gamma^{5} a_{\mu} N$ is a standard coupling of baryons to the axial vector current. In contrast, the gauge $W_{R}=1$ is not compatible with the transformation (5.9). (For this gauge there exists an alternative realization of $P$ and $C$ on the level of nonlinear fields. Under these modified $P$ and $C$ transformations $a_{\mu}$ and $v_{\mu}$ do not transform, however, as axial vectors and vectors, respectively. This can be seen from the identification $v_{\mu}=-a_{\mu}$ in this gauge.) Having found an appropriate gauge fixing for the reparametrization symmetry, we may now directly proceed to the analysis of the physical content of the effective action (4.7).

The kinetic term for the pseudoscalar mesons can be inferred from

$$
\begin{aligned}
\mathcal{L}_{\text {kin }}^{(P)} & =\left(\sigma_{0}^{2}+\frac{7}{36} \chi_{0}^{2}\right) \operatorname{Tr}\left\{\partial^{\mu} U^{\dagger} \partial_{\mu} U\right\}+\frac{1}{12} \chi_{0}^{2} \partial^{\mu} \theta \partial_{\mu} \theta \\
& =\frac{2}{f^{2}}\left(\sigma_{0}^{2}+\frac{7}{36} \chi_{0}^{2}\right) \partial^{\mu} \Pi^{z} \partial_{\mu} \Pi_{z}+\frac{1}{3}\left(\sigma_{0}^{2}+\frac{4}{9} \chi_{0}^{2}\right) \partial^{\mu} \theta \partial_{\mu} \theta
\end{aligned}
$$

Its standard normalization fixes a combination of $\sigma_{0}$ and $\chi_{0}$ in terms of the pseudoscalar decay constant $f$ which corresponds to an average in the octet [15] $f=\frac{2}{3} f_{K}+\frac{1}{3} f_{\pi}=106 \mathrm{MeV}$. For an optimal quantitative estimate of the expectation values $\sigma_{0}, \chi_{0}$ the partial Higgs effect should be included. As we show in appendix A, the mixing between the pseudoscalars contained in $U(\sim$ $i \bar{\psi} \psi)$ and those in the divergence of the axial-vector current $\left(\sim \partial_{\mu}\left(\bar{\psi} \gamma^{\mu} \gamma^{5} \psi\right)\right)$ introduces an additional negative contribution (partial Higgs effects)

$$
\Delta \mathcal{L}_{k i n}^{(P)}=-\frac{\Delta_{f}^{2}}{4} \operatorname{Tr}\left\{\partial^{\mu} \tilde{U}^{\dagger} \partial_{\mu} \tilde{U}\right\}-\Delta_{\theta}^{2} \partial^{\mu} \theta \partial_{\mu} \theta
$$

One infers a standard renormalization of the kinetic term for

$$
f^{2}=4 \sigma_{0}^{2}+\frac{7}{9} \chi_{0}^{2}-\Delta_{f}^{2}=\kappa_{f}^{2}\left(4 \sigma_{0}^{2}+\frac{7}{9} \chi^{2}\right)
$$

with

$$
\kappa_{f}=\left(1-\frac{\Delta_{f}^{2}}{4}\left(\sigma_{0}^{2}+\frac{7}{36} \chi_{0}^{2}\right)^{-1}\right)^{1 / 2}=\left(1+\frac{\Delta_{f}^{2}}{f^{2}}\right)^{-1 / 2} \leq 1
$$

The deviation of $\kappa_{f}$ from one is one of the most important effects of effective interactions not included in eq. (2.6). The effective interactions responsible for $\Delta_{f}^{2}$ also induce $S U(3)$-violating contributions to the pseudoscalar wave function renormalizations related to the strange quark mass ${ }^{\text {th }}$. This has led to a phenomenological estimate [15

$$
\Delta_{f}^{2} \approx 0.45 f^{2}, \kappa_{f} \approx 0.83
$$

\footnotetext{
${ }^{14}$ In the notation of ref. [15] one has $\Delta_{f}^{2} / f^{2}=-X_{\phi}^{-} \bar{\sigma}_{0}^{2} Z_{m}^{-1}$.
} 
The pseudoscalar decay constants provide now for a quantitative estimate of the expectation values $\sigma_{0}$ and $\chi_{0}$

$$
f_{0}=2 \sqrt{\sigma_{0}^{2}+\frac{7}{36} \chi_{0}^{2}}=f / \kappa_{f}=\left(\frac{2}{3} f_{K}+\frac{1}{3} f_{\pi}\right) / \kappa_{f}=128 \mathrm{MeV}
$$

In the approximation (2.6) of scalar Vervollständigung one should use the leading order relation $f=f_{0}$, keeping in mind that nonleading effects lower $f$ to its physical value.

We may also use this relation for a first estimate of the effective gauge coupling $g$. Let us denote the relative size of the octet and singlet contributions to the squared meson decay constant by

$$
x=\frac{7}{36} \frac{\chi_{0}^{2}}{\sigma_{0}^{2}} \quad, \quad \sigma_{0}=\frac{f_{0}}{2 \sqrt{1+x}}
$$

The combination of the relations (2.20) and (5.15) yields a bound for the effective gauge coupling $g$. From

$$
g=\frac{\sqrt{7}}{3}\left(\frac{1+x}{x}\right)^{1 / 2} \frac{\bar{M}_{\rho}}{f_{0}}=5.9\left(\frac{1+x}{x}\right)^{1 / 2}
$$

one infers $g>5.9$. If the octet condensate dominates (large $x$ ) the effective gauge coupling is $g \approx 6$.

The last field which needs a proper normalization is the $\eta^{\prime}$-meson. Including the partial Higgs effect (5.11), the kinetic term for the pseudoscalar singlet has the standard normalization for

$$
\begin{aligned}
\eta^{\prime} & =\left(\frac{2}{3}\left(\sigma_{0}^{2}+\frac{4}{9} \chi_{0}^{2}\right)-2 \Delta_{\theta}^{2}\right)^{1 / 2} \theta \\
& =\sqrt{\frac{2}{3}}\left(\sigma_{0}^{2}+\frac{4}{9} \chi_{0}^{2}\right)^{1 / 2} \kappa_{\theta} \theta
\end{aligned}
$$

(In the leading approximation (2.6) one should, of course, use $\kappa_{\theta}=1$.) Inserting (5.18) into (4.7) and expanding $\cos \theta$ in second order in $\theta$ one finds the mass of the $\eta^{\prime}$-meson

$$
M_{\eta^{\prime}}^{2}=\left(\frac{3}{2} \nu \sigma_{0}^{2}-\frac{1}{3} \nu^{\prime} \chi_{0}^{2}\right)\left(\sigma_{0}^{2}+\frac{4}{9} \chi_{0}^{2}\right)^{-1} \kappa_{\theta}^{-2} \sigma_{0}=\frac{3 f_{0}}{4 \kappa_{\theta}^{2} \sqrt{1+x}} \frac{\left(7 \nu-8 x \nu^{\prime}\right)}{(7+16 x)} .
$$

Its interactions can be extracted by inserting

$$
\theta=\frac{\sqrt{6}}{\kappa_{\theta} f_{0}}\left(\frac{1+x}{1+\frac{16}{7} x}\right)^{1 / 2} \eta^{\prime}=\frac{1}{H_{\eta^{\prime}}} \eta^{\prime}
$$

In the gauge (5.4) the nonlinear Lagrangian (4.7) can now be written in terms of the normalized physical fields $N, \tilde{V}_{\mu}, \Pi$ and $\eta^{\prime}$ as

$$
\mathcal{L}=\mathcal{L}_{N}+\mathcal{L}_{U}+\mathcal{L}_{V}
$$


with

$$
\begin{aligned}
\mathcal{L}_{N}= & i \operatorname{Tr}\left\{\bar{N}_{8} \gamma^{\mu}\left(\partial_{\mu}-i \gamma^{5} \tilde{a}_{\mu}+\frac{i}{6 H_{\eta^{\prime}}} \gamma^{5} \partial_{\mu} \eta^{\prime}\right) N_{8}\right\}+M_{8} \operatorname{Tr}\left\{\bar{N}_{8} \gamma^{5} N_{8}\right\} \\
& +i \bar{N}_{1} \gamma^{\mu}\left(\partial_{\mu}+\frac{i}{6 H_{\eta^{\prime}}} \gamma^{5} \partial_{\mu} \eta^{\prime}\right) N_{1}+M_{1} \bar{N}_{1} \gamma^{5} N_{1} \\
& +\frac{1}{\sqrt{3}} \operatorname{Tr}\left\{\bar{N}_{8} \gamma^{\mu} \gamma^{5} \tilde{a}_{\mu}\right\} N_{1}+\frac{1}{\sqrt{3}} \bar{N}_{1} \gamma^{\mu} \gamma^{5} \operatorname{Tr}\left\{\tilde{a}_{\mu} N_{8}\right\}
\end{aligned}
$$

Here we use the octet and singlet fields

$$
\begin{aligned}
& N_{1}=\frac{1}{\sqrt{3}} N, N_{8}=N-\frac{1}{3} \operatorname{Tr} N=N-\frac{1}{\sqrt{3}} N_{1}, \operatorname{Tr} N_{8}=0, \\
& \tilde{a}_{\mu}=a_{\mu}-\frac{1}{3} \operatorname{Tr} a_{\mu}=\frac{1}{2 f} \lambda_{z} \partial_{\mu} \Pi^{z}+O\left(\Pi^{3}\right), \operatorname{Tr} \tilde{a}_{\mu}=0 \\
& a_{\mu}=\tilde{a}_{\mu}-\frac{1}{6} \partial_{\mu} \theta=\tilde{a}_{\mu}-\frac{1}{6 H_{\eta^{\prime}}} \eta^{\prime}
\end{aligned}
$$

For the mesonic part one has

$$
\mathcal{L}_{U}=\frac{f^{2}}{4} \operatorname{Tr}\left\{\partial^{\mu} \tilde{U}^{\dagger} \partial_{\mu} \tilde{U}\right\}+\frac{1}{2} \partial^{\mu} \eta^{\prime} \partial_{\mu} \eta^{\prime}-M_{\eta^{\prime}}^{2} H_{\eta^{\prime}}^{2} \cos \left(\eta^{\prime} / H_{\eta^{\prime}}\right)
$$

with

$$
\tilde{U}=\tilde{\xi}^{2}=\exp \left(i \frac{\Pi^{z} \lambda_{z}}{f}\right), \operatorname{det} \tilde{U}=1
$$

and

$$
\begin{aligned}
\mathcal{L}_{V}= & \frac{1}{2} \operatorname{Tr}\left\{\tilde{V}^{\mu \nu} \tilde{V}_{\mu \nu}\right\}+\bar{M}_{\rho}^{2} \operatorname{Tr}\left\{\tilde{V}^{\mu} \tilde{V}_{\mu}\right\}+\frac{2}{g} \bar{M}_{\rho}^{2} \operatorname{Tr}\left\{\tilde{V}^{\mu} \tilde{v}_{\mu}\right\}+\frac{1}{g^{2}} \bar{M}_{\rho}^{2} \operatorname{Tr}\left\{\tilde{v}^{\mu} \tilde{v}_{\mu}\right\} \\
& -g \operatorname{Tr}\left\{\bar{N}_{8} \gamma^{\mu} N_{8} \tilde{V}_{\mu}\right\}-\frac{g}{\sqrt{3}}\left(\operatorname{Tr}\left\{\bar{N}_{8} \tilde{V}_{\mu}\right\} \gamma^{\mu} N_{1}+\bar{N}_{1} \gamma^{\mu} \operatorname{Tr}\left\{\tilde{V}_{\mu} N_{8}\right\}\right) \\
& -\operatorname{Tr}\left\{\bar{N}_{8} \gamma^{\mu} \tilde{v}_{\mu} N_{8}\right\}-\frac{1}{\sqrt{3}}\left(\operatorname{Tr}\left\{\bar{N}_{8} \tilde{v}_{\mu}\right\} \gamma^{\mu} N_{1}+\bar{N}_{1} \gamma^{\mu} \operatorname{Tr}\left\{\tilde{v}_{\mu} N_{8}\right\}\right)
\end{aligned}
$$

In the effective action (5.21) we have replaced the free parameters by $M_{8}, M_{1}$, $\bar{M}_{\rho}, M_{\eta^{\prime}}, f$ and $g$. We note that the number of parameters is reduced to six since only one particular combination of $\nu$ and $\nu^{\prime}$ appears in $M_{\eta^{\prime}}$. We recall that we know the order of magnitude of $g$ by eq. (5.17), which relates also $g$ to $x$. The proportionality constant $H_{\eta^{\prime}}$ appearing in the couplings of $\eta^{\prime}$ is then known by eq. (5.20). The interactions between physical baryons and mesons can be extracted by inserting the explicit representations

$$
\Pi=\frac{1}{2} \Pi^{z} \lambda_{z}=\frac{1}{2}\left(\begin{array}{ccc}
\pi^{0}+\frac{1}{\sqrt{3}} \eta, & \sqrt{2} \pi^{+}, & \sqrt{2} K^{+} \\
\sqrt{2} \pi^{-}, & -\pi^{0}+\frac{1}{\sqrt{3}} \eta & \sqrt{2} K^{0} \\
\sqrt{2} K^{-}, & \sqrt{2} \bar{K}^{0}, & -\frac{2}{\sqrt{3}} \eta
\end{array}\right)
$$




$$
\begin{gathered}
\tilde{V}_{\mu}=\frac{1}{2} \tilde{V}_{\mu}^{z} \lambda_{z}=\frac{1}{2}\left(\begin{array}{ccc}
\rho_{\mu}^{0}+\frac{1}{\sqrt{3}} V_{\mu}^{8}, & \sqrt{2} \rho_{\mu}^{+}, & \sqrt{2} K_{\mu}^{*+} \\
\sqrt{2} \rho_{\mu}^{-}, & -\rho_{\mu}^{0}+\frac{1}{\sqrt{3}} V_{\mu}^{8} & \sqrt{2} K_{\mu}^{* 0} \\
\sqrt{2} K_{\mu}^{*-}, & \sqrt{2} \bar{K}_{\mu}^{* 0}, & -\frac{2}{\sqrt{3}} V_{\mu}^{8}
\end{array}\right) \\
N_{8}=\left(\begin{array}{ccc}
\frac{1}{\sqrt{2}} \Sigma^{0}+\frac{1}{\sqrt{6}} \Lambda^{0}, & \Sigma^{+}, & p \\
\Sigma^{-}, & -\frac{1}{\sqrt{2}} \Sigma^{0}+\frac{1}{\sqrt{6}} \Lambda^{0}, & n \\
\Xi^{-}, & \Xi^{0}, & -\frac{2}{\sqrt{6}} \Lambda^{0}
\end{array}\right) \\
\bar{N}_{8}=\left(\begin{array}{ccc}
\frac{1}{\sqrt{2}} \bar{\Sigma}^{0}+\frac{1}{\sqrt{6}} \bar{\Lambda}^{0} . & \bar{\Sigma}^{-}, & \Xi^{-} \\
\bar{\Sigma}^{+}, & -\frac{1}{\sqrt{2}} \bar{\Sigma}^{0}+\frac{1}{\sqrt{6}} \bar{\Lambda}^{0}, & \bar{\Xi}^{0} \\
\bar{p}, & \bar{n}, & -\frac{2}{\sqrt{6}} \bar{\Lambda}^{0}
\end{array}\right)
\end{gathered}
$$

It is obvious that the effective action (5.21) predicts a multitude of different interactions between the low mass hadrons. The electromagnetic interactions are incorporated by covariant derivatives. We will discuss a few of these interactions in sects. 7-9 in order to check the validity of the hypothesis of scalar Vervollständigung.

Finally, the addition of the explicit chiral symmetry breaking by the current quark masses (2.9)

$$
\mathcal{L}_{j}=-\frac{1}{2} Z_{\phi}^{-1 / 2} a_{q} \sigma_{0} \operatorname{Tr}\left\{\bar{m}\left(U+U^{\dagger}\right)\right\}
$$

leads to mass terms for the pseudoscalars [16] and contributes to their interactions.

\section{Baryon number}

The correct assignment of the baryon number $B$ is the central question for quark-baryon duality. Quarks carry $B=1 / 3$, whereas baryons have $B=1$. How can this be reconciled with a dual picture where one field describes quarks as well as baryons in the appropriate momentum ranges? In principle, gluon-meson duality (or the Higgs picture of QCD) is consistent with two alternatives. In the first scenario the nonlinear fermion fields $N$ could transform as octets with integer charge and $B=1 / 3$. In this case separate baryon fields would be needed, with all quantum numbers identical to the quark fields except for the baryon number. As in the nonrelativistic quark model or the parton model a baryon field may then be thought of as the composite of three quark fields. An important puzzle would remain, however, in this scenario: Why should the color singlet integer charged fermion octet field $N$ not be associated to physical particles? We will argue here in favor of the second alternative, namely that the nonlinear field $N$ indeed carries $B=1$ (and not $B=1 / 3$ ). It can therefore be associated directly with the baryons. Possible relations of this picture of quark-baryon duality with the nonrelativistic quark model will be discussed in this section and 
sect. 11. The crucial ingredient for the viability of this second scenario are the nontrivial transformation properties of the various nonlinear fields.

The determination of the baryon number of the nonlinear fields needs some care. To get familiar with the problem, we first discuss the analogous problem for the electric charge. The equivalence between the nonlinear language and the Higgs picture discussed in sect. 3 requires the nonlinear fields in $v$ to carry electric charge. Indeed, the transformation laws

$$
\delta_{e m} \psi=i \beta \tilde{Q} \psi, \delta_{e m} \tilde{B}_{\mu}=\frac{1}{\tilde{e}} \partial_{\mu} \beta, \delta_{e m} W_{L, R}=i \beta\left[\tilde{Q}, W_{L, R}\right], \delta_{e m} v=i \beta \tilde{Q} v
$$

guarantee that the quarks carry fractional electric charge $\tilde{Q}=\operatorname{diag}\left(\frac{2}{3},-\frac{1}{3},-\frac{1}{3}\right)$ and the gluons are neutral, whereas the pions in $\xi$, the baryons and the vector mesons are integer charged

$$
\delta_{e m} \xi=i \beta[\tilde{Q}, \xi], \delta_{e m} N=i \beta[\tilde{Q}, N], \delta_{e m} V_{\mu}=i \beta\left[\tilde{Q}, V_{\mu}\right]+\frac{1}{g} \partial_{\mu} \beta \tilde{Q}
$$

Despite the fact that $v$ does not appear in the effective action (4.10), its nontrivial electric charge plays a crucial role in the transition from the linear quark-gluon to the nonlinear baryon-meson description.

The situation is similar for the baryon number, which we denote by $\tilde{B}$ on the quark level, $\tilde{B}(\psi)=1 / 3$. The field $v^{\dagger}$ transforms as a color antitriplet. By triality it should therefore carry baryon number $\tilde{B}=2 / 3$. The baryon number, electric charge and color transformations of $v^{\dagger}$ coincide all with the ones for a diquark field. As a simple consequence, the baryons $N$ carry $\tilde{B}=1$ as it should be, consistent with triality. (Triality requires that the $S U(3)_{C}$ representations in the classes $(1,8, \ldots),(3, \overline{6}, \ldots),(\overline{3}, 6, \ldots)$ carry $\tilde{B}=0, \frac{1}{3}, \frac{2}{3} \bmod 1$.) We note that $\chi$ is bilinear in $v$ and $v^{\dagger}$ and therefore carries $\tilde{B}=0$ as appropriate for a quark-antiquark composite. Besides the above consistency considerations a direct determination of the baryon number for $v$ becomes easy if some scalar fields are linear in $v$. In sect. 11 we will see how the association of $v^{\dagger}$ with a nonlinear diquark field arises naturally in a language where linear diquark fields are introduced.

Let us next give the equivalent description of baryon number in the Higgs picture. In a language with gauge-fixed reparametrization invariance the condition $v=1$ preserves a combination of $U(1)_{P}$ and baryon number transformation, $2 B_{P}(v)+\tilde{B}(v)=0$. Furthermore, $W_{L}$ and $W_{R}$ are neutral with respect to the abelian charge $B_{P}+B_{V}$ (cf. eq. (4.4). After spontaneous symmetry breaking the unbroken baryon number-type charge is therefore

$$
B=\tilde{B}+2 B_{P}+2 B_{V}
$$

Both $\psi$ and $N$ have the same charge $B=1$. The Higgs picture implies that the "physical baryon number" $B$ after spontaneous symmetry breaking 
is three times the quark baryon number $\tilde{B}$. In table 2 we have listed the various abelian charges for the linear and nonlinear fields, including the charge $B_{N}$ (cf. eq. (4.4)). We note the linear relation $\tilde{B}=B_{P}+B_{V}+B_{N}$.

\begin{tabular}{c|cccccc} 
& $\tilde{B}$ & $B_{P}$ & $\tilde{B}+2 B_{P}$ & $B_{V}$ & $B$ & $B_{N}$ \\
\hline$\psi$ & $\frac{1}{3}$ & 0 & $\frac{1}{3}$ & $\frac{1}{3}$ & 1 & 0 \\
$v^{\dagger}$ & $\frac{2}{3}$ & $-\frac{1}{3}$ & 0 & 0 & 0 & 1 \\
$N$ & 1 & 0 & 1 & 0 & 1 & 1 \\
$W_{L, R}$ & 0 & $-\frac{1}{3}$ & $-\frac{2}{3}$ & $\frac{1}{3}$ & 0 & 0 \\
$U, S, X$ & 0 & 0 & 0 & 0 & 0 & 0 \\
$A_{\mu}, V_{\mu}$ & 0 & 0 & 0 & 0 & 0 & 0 \\
\hline & & & & &
\end{tabular}

Table 2: Abelian charges of linear and nonlinear fields

In conclusion, the equivalence between the Higgs picture and the nonlinear language with preserved local symmetries shows many similarities between baryon number and electric charge. One difference remains, however, in the Higgs picture. Whereas some "linear" scalar expectation values $\chi$ carry nonzero electric charge $\tilde{Q}$ (cf. eq. (3.3) they have all $\tilde{B}=0$. The "spontaneous breaking" of $\tilde{B}$ occurs only on the level of the nonlinear fields $v$ and $W_{L, R}$ by the gauge fixing $v=1, W_{L}^{\dagger}=W_{R}$.

Let us next turn to the normalization of the fermion fields $\psi$ and $N$ and the associated relative wave function renormalization $Z_{\psi}$. In principle, we may normalize the fields according to some conserved charge or to some standard convention for the kinetic term. For the quark field $\psi$ we choose a normalization such that the quark number operator $N_{q}$ has a standard form

$$
N_{q}=-i \int d^{3} x \operatorname{Tr} \bar{\psi} \gamma^{0} \psi
$$

More precisely, the normalization specifies the way how a baryon-chemical potential $\mu_{B}$ is introduced for situations with nonvanishing baryon density. Since quarks carry $\tilde{B}=1 / 3$, we take

$$
\mathcal{L}^{(\mu)}=\frac{i}{3} \mu_{B} \operatorname{Tr} \bar{\psi} \gamma^{0} \psi
$$

On the other hand, we have normalized the field $N$ such that its kinetic term has a standard form $\square$ (i.e. $Z_{N}=1$ ). Since the baryons are "physical

\footnotetext{
${ }^{15}$ Note that the normalization of $B$ is fixed by the requirement that a hypothetical particle carrying baryon number without strong interactions (and therefore $B_{V}=B_{P}=0$ ) should have $B=\tilde{B}$.

${ }^{16}$ This should hold at least in the vicinity of on-shell momenta.
} 
fields", we assume that this also corresponds to a standard normalization of the baryon number operator

$$
N_{B}=-i \int d^{3} x \operatorname{Tr} \bar{N} \gamma^{0} N
$$

such that

$$
\mathcal{L}^{(\mu)}=i \mu_{B} \operatorname{Tr} \bar{N} \gamma^{0} N
$$

The consistency of eqs. (6.5) and (6.7) with the definition of the nonlinear field $N$ (4.1) requires

$$
Z_{\psi}=\frac{1}{3}
$$

This reflects in our language the fact that baryons carry three times the baryon number of quarks. We emphasize that the choice $Z_{\psi}=1 / 3$ is not a pure convention but rather a necessity if $\psi$ and $N$ are normalized according to (6.5), (6.7). Quark-baryon duality has then the interesting consequence of a nontrivial wave function renormalization $Z_{\psi}$ in the "quark number normalization", since the kinetic terms for $\psi$ and $N$ are directly related.

The wave function renormalization $Z_{\psi}$ is related to the full quantum theory in the low momentum limit, $Z_{\psi} \equiv Z_{\psi}(k \rightarrow 0)=1 / 3$. In contrast, we expect that for large $k$ a perturbative picture is valid, with $Z_{\psi}(k \gg 2 \mathrm{GeV})$ $\approx 1$. This leads to a simple speculation how quark-baryon duality can be reconciled with the linear quark-meson model [17], [13], [15] the nonrelativistic quark model and the parton model. The quark model descriptions are valid in the range of large enough $k$ (or large momenta) where $Z_{\psi}(k) \approx 1$. We speculate that around some scale $k_{B}$ the wave function renormalization $Z_{\psi}(k)$ drops rapidly to its nonperturbative value $Z_{\psi}(k \rightarrow 0)=1 / 3$. This rapid drop is related to the binding of three quarks to a baryon in the nonrelativistic quark model. We therefore associate $k_{B}$ to a characteristic scale where this binding takes place. The fact that $Z_{\psi}$ drops precisely to the value $1 / 3$ reflects the fact that three quarks are needed for a baryon in the nonrelativistic quark model.

We may further speculate that the "binding effect" essentially only affects the quark wave function renormalization $Z_{\psi}$ and other wave function renormalizations related to it by Ward identities. This has the interesting consequence that the drop in $Z_{\psi}$ at $k_{B}$ results in a corresponding increase of those renormalized couplings which involve negative powers of $Z_{\psi}$. As an example, one may take the Yukawa couplings $h, \tilde{h}$ in the effective action (2.6). If the "unrenormalized couplings" $Z_{\psi} h$ and $Z_{\psi} \tilde{h}$ do not undergo a major change near $k_{B}$ the Yukawa couplings will increase rapidly by a factor three around this scale. Neglecting the running of $Z_{\psi} h$ one obtains $h\left(k \gtrsim k_{B}\right)=\frac{1}{3} h(k=0)$ and similar for $\tilde{h}$. If also the expectation values $\sigma_{0}(k)$ and $\chi_{0}(k)$ do not vary much in this range, the effective fermion masses for $k \gtrsim k_{B}$ are one third the baryon masses. This is characteristic for models with consituent quarks. A particular version of such models, the linear quark meson model, has given a 
rather satisfactory description of chiral symmetry breaking for two flavors of light quarks 13]. The relevant range for its dynamics is $700 \mathrm{MeV}>k>k_{B}$, and one may wonder how it is related precisely to our scenario for $k=0$.

On the other hand, it has been pointed out [18] that fluctuations with momenta in the range $0<k<k_{B}$ play an important role for the chiral properties at large baryon density. The transition from effective nucleon degrees of freedom to quark degrees of freedom is directly linked to the phase transition from a hadron gas to nuclear matter at vanishing or low temperature. A change of $Z_{\psi}$ by a factor of three would precisely account for the enhancement of the contribution from baryon fluctuations as compared to quark fluctuations to the dependence of the effective potential on the baryon chemical potential [18.

Another interesting quantity is the running renormalized gauge coupling $g(k)$. If we push perturbation theory to its limit we obtain

$$
g(850 \mathrm{MeV})=\sqrt{4 \pi \alpha_{s}(850 \mathrm{MeV})}=2.26
$$

where the numerical value corresponds to the two-loop value in the $\overline{M S}$ scheme ${ }^{\text {I7 }}$. Neglecting the running of $Z_{\psi} g$ for scales below the mass of the vector mesons and accounting for a drop of $Z_{\psi}$ by a factor of three at $k_{B}$, this yields the "perturbative estimates"

$$
\begin{array}{ll}
g^{(p t)}=6.8 & , \quad \chi_{0}^{(p t)}=125 \mathrm{MeV} \\
x^{(p t)}=3.1 & , \quad \sigma_{0}^{(p t)}=31 \mathrm{MeV}
\end{array}
$$

With such an estimate the free parameters of our simple model would be fixed in terms of $f$ and $\bar{M}_{\rho}$.

In order to test the consistency of these ideas, it is instructive to couple a hypothetical massless "baryophoton" $\tilde{C}_{\mu}$ to the quarks and baryons. This would be needed if the abelian symmetry corresponding to baryon number is gauged [8]. According to the "quark number" normalization (6.4), the coupling of the hypothetical "baryophoton" is

$$
\mathcal{L}^{(C)}=\frac{1}{3} e_{B} \operatorname{Tr} \bar{\psi} \gamma^{\mu} \psi \tilde{C}_{\mu}
$$

with $e_{B}$ some arbitrarily small gauge coupling which may depend on $k$. With $Z_{\psi}=1 / 3$ for $k=0$ this corresponds to a covariant derivative $\partial_{\mu}-i e_{B} \tilde{C}_{\mu}$ for the quark field $\psi$. Inserting the relation (4.1) between $\psi$ and $N$, the coupling (6.11) becomes

$$
\mathcal{L}^{(C)}=\frac{1}{3 Z_{\psi}} e_{B} \operatorname{Tr} \bar{N} \gamma^{\mu} N \tilde{C}_{\mu}
$$

\footnotetext{
${ }^{17}$ We use $\Lambda_{\mathrm{QCD}}=250 \mathrm{MeV}$. The variation of $g$ in this momentum region is still moderate, with $g(1 \mathrm{GeV})=2.12, g(700 \mathrm{MeV})=2.48$.

${ }^{18} \mathrm{In}$ absence of weak interactions baryon number is free of anomalies and permits therefore a consistent local gauge symmetry.
} 
For $3 Z_{\psi}=1$ this is indeed the expected coupling of the "baryophoton" to a baryon (cf. eq. (6.6)).

In conclusion, quark-baryon duality may well be compatible with a picture where baryons are composed of three quarks. At least we have found no obvious contradiction. The quantum numbers match. A crucial ingredient for dynamical considerations seems to be the value $Z_{\psi}=1 / 3$ for $k \rightarrow 0$ and $Z_{\psi} \approx 1$ for $k \gg k_{B}$. A more detailed dynamical understanding why and how the binding of three quarks to a baryon in the nonrelativistic quark model is related to the drop of $Z_{\psi}$ by a factor $1 / 3$ in the language of quarkbaryon duality would be of great value. For the present paper we take this as a working hypothesis and explore phenomenological consequences of quarkbaryon duality. A discussion of linear diquark fields in sect. 11 will shed a little more light on this issue.

\section{$7 \quad$ Electromagnetic interactions}

As a first probe of our picture we may use the electromagnetic interactions of mesons and baryons. The electromagnetic interactions of the mesons are all contained in the covariant kinetic term for the scalars

$$
\begin{aligned}
\left(D^{\mu} \gamma_{i j, a b}\right)^{*} D_{\mu} \gamma_{i j, a b}= & \chi_{0}^{2} \operatorname{Tr}\left\{\left(\hat{v}^{\mu}+g \tilde{V}^{\mu}-\tilde{e} \tilde{B}^{\mu} \tilde{Q}\right)\left(\hat{v}_{\mu}+g \tilde{V}_{\mu}-\tilde{e} \tilde{B}_{\mu} \tilde{Q}\right)\right\} \\
& +\frac{f^{2}}{4} \operatorname{Tr}\left\{\left(D^{\mu} U\right)^{\dagger} D_{\mu} U\right\}+\frac{1}{12} \chi_{0}^{2} \partial^{\mu} \theta \partial_{\mu} \theta
\end{aligned}
$$

Here $D_{\mu} U=\partial_{\mu} U-i \tilde{e} \tilde{B}_{\mu}[\tilde{Q}, U]$ and the covariant vector current reads (with $\left.\tilde{D}_{\mu} \xi=\partial_{\mu} \xi-i \tilde{e} \tilde{B}_{\mu}[\tilde{Q}, \xi]\right)$

$$
\begin{aligned}
\hat{v}_{\mu} & =-\frac{i}{2}\left(W_{L}^{\dagger} \tilde{D}_{\mu} W_{L}+W_{R}^{\dagger} \tilde{D}_{\mu} W_{R}\right) \\
& =-\frac{i}{2}\left(\xi^{\dagger} \tilde{D}_{\mu} \xi+\xi \tilde{D}_{\mu} \xi^{\dagger}\right) \\
& =v_{\mu}-\frac{\tilde{e}}{2} \tilde{B}_{\mu}\left(\xi^{\dagger} \tilde{Q} \xi+\xi \tilde{Q} \xi^{\dagger}-2 \tilde{Q}\right)
\end{aligned}
$$

Observing that both $\hat{v}_{\mu}$ and $g \tilde{V}_{\mu}-\tilde{e} \tilde{B}_{\mu} \tilde{Q}$ transform homogeneously with respect to the electromagnetic gauge transformations

$$
\delta_{e m} \hat{v}_{\mu}=i \beta\left[\tilde{Q}, \hat{v}_{\mu}\right], \delta_{e m}\left(g \tilde{V}_{\mu}-\tilde{e} \tilde{B}_{\mu} \tilde{Q}\right)=i \beta\left[Q,\left(g \tilde{V}_{\mu}-\tilde{e} \tilde{B}_{\mu} \tilde{Q}\right)\right]
$$

the gauge invariance of (7.1) can be easily checked. The particular combination of vector currents in (7.1) is dictated by the combination of electromagnetic gauge invariance and local reparametrization symmetry. Only this combination transforms homogeneously with respect to both $U(3)_{P}$ local reparametrizations and electromagnetic $U(1)$ gauge transformations. In particular, one has

$\delta\left(\hat{v}_{\mu}-\tilde{e} \tilde{B}_{\mu} \tilde{Q}\right)=i\left[\Theta_{P},\left(\hat{v}_{\mu}-\tilde{e} \tilde{B}_{\mu} \tilde{Q}\right)\right]+i \tilde{e} \tilde{B}_{\mu}\left[\tilde{Q},\left(\hat{v}_{\mu}-\tilde{e} \tilde{B}_{\mu} \tilde{Q}\right)\right]-\partial_{\mu} \Theta_{P}-\partial_{\mu} \beta \tilde{Q}$ 
The interactions (7.1) coincide with those of a "hidden local chiral symmetry" 19] if one replaces $\hat{v}_{\mu}$ by $v_{\mu}$. The difference between our result and the "hidden symmetry" approach is due to the different electromagnetic transformation properties, cf. eqs. (6.1), (6.2). If one restricts the discussion to $\rho$-mesons and pions, $\tilde{V}_{\mu}=\frac{1}{2} \vec{\rho}_{V \mu} \vec{\tau}, \Pi=\frac{1}{2} \vec{\pi} \vec{\tau}$, and neglects the difference between $\tilde{e}$ and $e$, one finds for the term involving the vector mesons and currents

$$
\begin{aligned}
\mathcal{L}_{V V}= & a f_{\pi}^{2} \operatorname{Tr}\left(\hat{v}_{\mu}+\frac{1}{2} g_{\rho} \vec{\rho}_{V \mu} \vec{\tau}-\frac{1}{2} e \tilde{B}_{\mu} \tau_{3}\right)^{2} \\
= & a f_{\pi}^{2} \operatorname{Tr}\left[\frac{1}{4 f_{\pi}^{2}}\left(\left(\vec{\pi} \times \partial_{\mu} \vec{\pi}\right) \vec{\tau}\right)+\frac{1}{2} g_{\rho}\left(\vec{\rho}_{V \mu} \vec{\tau}\right)-\frac{1}{2} e \tilde{B}_{\mu} \tau_{3}\right. \\
& \left.-\frac{e}{4 f_{\pi}^{2}} \tilde{B}_{\mu}\left(\pi_{3}(\vec{\pi} \vec{\tau})-(\vec{\pi} \vec{\pi}) \tau_{3}\right)\right]^{2}+\ldots
\end{aligned}
$$

For the second equality in eq. (7.5) we have only retained terms quadratic in $\pi$ in an expansion of $\hat{v}_{\mu}$ and we have replaced $f$ by $f_{\pi}$. The first expression in eq. (7.5) is actually more general than the result of the particular effective action (2.6) for which one has

$$
\begin{aligned}
g_{\rho} & =g \\
a & =\frac{\chi_{0}^{2}}{f_{\pi}^{2}}=\frac{9}{7 \kappa_{f}^{2}} \frac{x}{1+x} \frac{f^{2}}{f_{\pi}^{2}} \approx 2.4 \frac{x}{1+x}
\end{aligned}
$$

In consequence of the symmetries, additional interactions will change the values of a $a$ and $g_{\rho}$ without affecting the structure of the invariant. Such additional interactions will also generate new invariants involving higher derivatives. We give an example in appendix A.

The contributions in eq (7.5) with canonical dimension $\leq 4$ can be written in the form

$$
\begin{aligned}
\mathcal{L}_{V V}= & \frac{1}{2} M_{\rho}^{2} \vec{\rho}_{V}^{\mu} \vec{\rho}_{V \mu}-e g_{\rho \gamma} \rho_{V 3}^{\mu} \tilde{B}_{\mu}+\frac{1}{2} m_{B}^{2} \tilde{B}^{\mu} \tilde{B}_{\mu} \\
& +g_{\rho \pi \pi} \vec{\rho}_{V}^{\mu}\left(\vec{\pi} \times \partial_{\mu} \vec{\pi}\right)+g_{\gamma \pi \pi}^{(V)} \tilde{B}^{\mu}\left(\vec{\pi} \times \partial_{\mu} \vec{\pi}\right)_{3} \\
& +g_{\rho \gamma \pi \pi} \tilde{B}_{\mu}\left[(\vec{\pi} \vec{\pi}) \rho_{V 3}^{\mu}-\left(\vec{\rho}_{V}^{\mu} \vec{\pi}\right) \pi_{3}\right] \\
& -\frac{1}{2} a e^{2} \tilde{B}_{\mu} \tilde{B}^{\mu}\left[\left(\vec{\pi} \vec{\pi}-\pi_{3}^{2}\right]+\ldots\right.
\end{aligned}
$$

with

$$
\begin{aligned}
& M_{\rho}^{2}=a g_{\rho}^{2} f_{\pi}^{2}, g_{\rho \gamma}=a g_{\rho} f_{\pi}^{2}, g_{\rho \pi \pi}=\frac{1}{2} a g_{\rho}, \\
& m_{B}^{2}=a e^{2} f_{\pi}^{2}, g_{\gamma \pi \pi}^{(V)}=-\frac{1}{2} a e, g_{\rho \gamma \pi \pi}=\frac{1}{2} a e g_{\rho}
\end{aligned}
$$

We recover the very successful KSFR relation [20], [19]

$$
g_{\rho \gamma}=2 f_{\pi}^{2} g_{\rho \pi \pi}
$$


which relates the decay $\rho \rightarrow 2 \pi$ (with $g_{\rho \pi \pi} \approx 6$, see next section) to the eletromagnetic properties of the $\rho$-meson, in particular the decay $\rho_{0} \rightarrow e^{+} e^{-}$ (with $\Gamma\left(\rho_{0} \rightarrow e^{+} e^{-}\right)=6.62 \mathrm{keV}$ and $g_{\rho \gamma}=0.12 \mathrm{GeV}^{2}$ ).

The relation

$$
M_{\rho}^{2}=\frac{4}{a} g_{\rho \pi \pi}^{2} f_{\pi}^{2}
$$

requires $a=2.1$, implying

$$
\chi_{0}=135 \mathrm{MeV}
$$

One may use eq. (7.6) for an estimate of the relative octet contribution to the pion decay constant and eq. (2.20) or (5.19) for an estimate of $g$

$$
x=7 \quad, \quad \sigma_{0}=22 \mathrm{MeV} \quad, \quad g=6.3
$$

It is striking how close these values are to the "perturbative values" (6.10). This should, however, not be taken too literally in view of possible substantial $S U(3)_{V}$-violating effects from the nonzero strange quark mass. Also higher order operators may affect the relation (7.6). Furthermore, one should include the corrections from additional invariants (see appendix A). A phenomenological discussion including the properties of the axial-vector mesons [21] favors $a \approx 1.64$. Relation (7.6) implies then

$$
x=2.16 \quad, \quad \chi_{0}=119 \mathrm{MeV} \quad, \quad \sigma_{0}=36 \mathrm{MeV} \quad, \quad g=7.1
$$

again close to the "perturbative values" (6.10). We point out that the minimal effective action (2.6) with $\kappa_{f}=1$ and $f_{\pi}=f$ leads to a bound $a<9 / 7$. We will discuss this issue and modifications of the relation (7.6) in the next section and appendix A. The additional invariants encountered in appendix A only affect the cubic and higher vertices, but not the mass terms. In particular, the relation

$$
g_{\rho \gamma}=g \chi_{0}^{2}=\frac{\bar{M}_{\rho}^{2}}{g}
$$

will only be modified by $S U(3)$-violating effects. It can be used for an independent estimate of $g$, yielding

$$
g=6
$$

close to (7.12) and the saturation of the bound implied by eq. (5.17).

The electromagnetic $\gamma \pi \pi$ and $\gamma \gamma \pi \pi$ vertices also receive contributions from

$$
\begin{aligned}
\frac{1}{4} f_{\pi}^{2} \operatorname{Tr}\left\{D^{\mu} U^{\dagger} D_{\mu} U\right\}= & \frac{1}{2} \partial^{\mu} \vec{\pi} \partial_{\mu} \vec{\pi} \\
& +e \tilde{B}^{\mu}\left(\vec{\pi} \times \partial_{\mu} \vec{\pi}\right)_{3}+\frac{1}{2} e^{2} \tilde{B}_{\mu} \tilde{B}^{\mu}\left(\vec{\pi} \vec{\pi}-\pi_{3}^{2}\right)+\ldots
\end{aligned}
$$

One sees that for $a=2$ the two contributions (7.7) and (7.16) to the direct $\gamma \pi \pi$ vertex cancel. The electromagnetic interactions of the pions are then 
dominated by $\rho$-exchange (vector dominance), in agreement with observation. Otherwise stated, our model leads for the direct $\gamma \pi \pi$-coupling to the realistic relation

$$
g_{\gamma \pi \pi}=e\left(1-\frac{2 g_{\rho \pi \pi}^{2} f_{\pi}^{2}}{M_{\rho}^{2}}\right)
$$

The vertex $\sim g_{\rho \gamma \pi \pi}$ contributes[ ${ }^{\mathbb{P}}$ to rare decays like $\rho_{0} \rightarrow \pi^{+} \pi^{-} \gamma$, with

$$
g_{\rho \gamma \pi \pi}=e g_{\rho \pi \pi}
$$

We conclude that the electromagnetic interactions of the pseudoscalars as well as the vector mesons can be considered as a successful test of our simple model. The appearance of a local nonlinear reparametrization symmetry is a direct consequence of the "spontaneous breaking" of color. This symmetry, combined with the simple effective action (2.6), has led to the KSFR relation (7.9) and to vector dominance (7.17). At this stage the relations of the above discussion should be taken with a 20-30 percent uncertainty. In particular, the $S U(3)$-violation due to the nonzero strange quark mass needs to be dealt with more carefully. Nevertheless, the $\rho \rightarrow 2 \pi$ decay and the electromagnetic decays are all consistent and have allowed us a first determination of the size of the octet condensate $\chi_{0}$, which we may take in the range between the estimates (7.11) and (7.13), i.e. $119 \mathrm{MeV}<\chi_{0}<135 \mathrm{MeV}$. The octet condensate is larger than the singlet condensate and dominates the pseudoscalar decay constant $f$.

Finally, the connection of the above discussion to the Higgs picture developed in sect. 3 is easily established if one realizes that the field

$$
\begin{aligned}
G_{\mu} & =-\sqrt{3} \operatorname{Tr}\left\{\tilde{Q} \tilde{V}_{\mu}\right\}=\sqrt{3} \operatorname{Tr}\left\{\tilde{Q}\left(v A_{\mu}^{T} v^{\dagger}+\frac{i}{g} \partial_{\mu} v v^{\dagger}\right)\right\} \\
& =-\left(\frac{\sqrt{3}}{2} \rho_{\mu}^{0}+\frac{1}{2} V_{\mu}^{8}\right)
\end{aligned}
$$

is the nonlinear correspondence of $\tilde{G}_{\mu}$ in sect. 3. It transforms inhomogeneously

$$
\delta_{e m} G_{\mu}=-\frac{2}{\sqrt{3} g} \partial_{\mu} \beta=-\frac{1}{\tilde{g}} \partial_{\mu} \beta
$$

such that the linear combinations (3.7) $R_{\mu}=\cos \theta_{e m} G_{\mu}+\sin \theta_{e m} \tilde{B}_{\mu}, B_{\mu}=$ $\cos \theta_{e m} \tilde{B}_{\mu}-\sin \theta_{e m} G_{\mu}$ have the transformation properties of a heavy neutral boson and a photon

$$
\delta_{e m} B_{\mu}=\frac{1}{e} \partial_{\mu} \beta, \delta_{e m} R_{\mu}=0
$$

Inserting in $\mathcal{L}_{V}$ (5.26) $\tilde{V}_{\mu}=-\frac{\sqrt{3}}{2} G_{\mu} \tilde{Q}, \tilde{v}_{\mu}=0$ and adding terms from covariant derivatives (3.1) involving $\tilde{B}_{\mu}$ (cf. (7.5)), we recover $\mathcal{L}_{\text {em }}^{(2)}$ (3.6) with $\tilde{G}_{\mu}$

\footnotetext{
${ }^{19}$ This vertex is absent in ref. [19].
} 
replaced by $G_{\mu}$. The (extended) electromagnetic interactions of the baryon octet $20 \mathrm{read}$

$$
\begin{aligned}
\mathcal{L}_{V N, 0}= & e B_{\mu} \operatorname{Tr}\left\{\bar{N}_{8} \gamma^{\mu}\left[\tilde{Q}, N_{8}\right]\right\} \\
& +\tilde{g} \cos \theta_{e m} R_{\mu} \operatorname{Tr}\left\{\bar{N}_{8} \gamma^{\mu}\left(N_{8} \tilde{Q}+t g^{2} \theta_{e m} \tilde{Q} N_{8}\right)\right\}
\end{aligned}
$$

One finds the standard coupling between the photon $B_{\mu}$ and the baryons according to their charge. More generally, we conclude from

$$
\begin{aligned}
& \tilde{e} \tilde{B}_{\mu}=e\left(B_{\mu}+t g \theta_{e m} R_{\mu}\right) \\
& \tilde{g} G_{\mu}=\tilde{g} \cos \theta_{e m} R_{\mu}-e B_{\mu}
\end{aligned}
$$

that the charged leptons have a small direct coupling to a linear combination of the $\rho^{0}, \omega$ and $\phi$ vector mesons corresponding to the term $e \operatorname{tg} \theta_{e m} R_{\mu}$ in $\tilde{e} \tilde{B}_{\mu}$. Similarly, the photon has a hadronic coupling from the term $-e B_{\mu}$ in $\tilde{g} G_{\mu}$. These mixing effects are governed by the coupling $g_{\gamma \rho}(7.9)$.

\section{Vector mesons}

The vector mesons acquire a mass through the Higgs mechanism. They are also unstable due to the decay into two pseudoscalar mesons. Their interactions are contained in $\mathcal{L}_{V}$ (5.26). In particular, the cubic vertex between one vector meson and two pseudoscalars is

$$
\begin{aligned}
\mathcal{L}_{V \pi \pi} & =2 g \chi_{0}^{2} \operatorname{Tr}\left\{\tilde{V}^{\mu} \tilde{v}_{\mu}\right\}=-\frac{i g \chi_{0}^{2}}{f^{2}} \operatorname{Tr}\left\{\left[\Pi, \partial_{\mu} \Pi\right] \tilde{V}^{\mu}\right\} \\
& =-2 i g_{\rho \pi \pi} \operatorname{Tr}\left\{\left[\Pi, \partial_{\mu} \Pi\right] \tilde{V}^{\mu}\right\}
\end{aligned}
$$

with

$$
g_{\rho \pi \pi}=\frac{g}{2} \frac{\chi_{0}^{2}}{f^{2}}=\frac{M_{\rho}^{2}}{2 g f^{2}}
$$

Considering only the effective action (2.6), one has $\kappa_{f}=1$ and infers

$$
g_{\rho \pi \pi}=\frac{9 x}{14(1+x)} g \approx 4.6\left(\frac{x}{1+x}\right)^{1 / 2} .
$$

If we restrict these interactions to the $\rho$-mesons $\tilde{V}_{\mu}=\frac{1}{2} \vec{\rho}_{V \mu} \vec{\tau}$ and pions $\Pi=$ $\frac{1}{2} \vec{\pi} \vec{\tau}, v_{\mu}=\frac{1}{4 f^{2}}\left(\vec{\pi} \times \partial_{\mu} \vec{\pi}\right) \vec{\tau}$, we obtain the familiar form

$$
\mathcal{L}_{\rho \pi \pi}=\frac{g \chi_{0}^{2}}{2 f^{2}} \epsilon_{i j k} \pi^{i} \partial_{\mu} \pi^{j} \rho_{V}^{k \mu}=g_{\rho \pi \pi}\left(\vec{\pi} \times \partial_{\mu} \vec{\pi}\right) \vec{\rho}_{V}^{\mu}
$$

\footnotetext{
${ }^{20}$ We omit here terms involving the baryon singlet $N_{1}$ and take $\Pi=0$.
} 
It is straightforward to compute the decay rate $\rho \rightarrow 2 \pi$ as

$$
\Gamma(\rho \rightarrow \pi \pi)=\frac{g_{\rho \pi \pi}^{2}}{48 \pi} \frac{\left(M_{\rho}^{2}-4 M_{\pi}^{2}\right)^{3 / 2}}{M_{\rho}^{2}} \simeq 150 \mathrm{MeV}
$$

and one infers the phenomenological value $g_{\rho \pi \pi} \simeq 6.0$. The discrepancy with eq. (8.3) reflects the difference between the realistic value $a \approx 2$ and a value of $a$ which results from (7.6) for $\kappa_{f}=1, f_{\pi}=f$. The estimate (8.3) receives, however, important corrections. Omitted $S U(3)_{V}$-violating effects from the strange quark mass result in corrections $\sim 30 \%$. Furthermore, since eq. (7.6) is no symmetry relation, it is subject to modifications from the inclusion of additional fields. (We have already included in eq. (7.6) the presumably most important modification in the form of $\kappa_{f}<1$.)

The effective coupling between $\rho$-mesons and baryons (5.26) obeys

$$
\mathcal{L}_{\bar{N} N_{\rho}}=-\frac{g}{2} \operatorname{Tr}\left\{\bar{N}_{8} \gamma^{\mu} N_{8} \vec{\tau}\right\} \vec{\rho}_{V \mu}
$$

where we omit from now on electromagnetic effects. This implies that the effective action (2.6) does not contain a direct coupling of the $\rho$-mesons to protons and neutrons. The $\rho$-mesons only couple to strange baryons. In the approximation of the effective action (2.6) possible contributions to the nucleon-nucleon interactions in the isospin triplet vector channel could only arise through two-pion interactions $\sim \operatorname{Tr}\left\{\bar{N}_{8} \gamma^{\mu} \tilde{v}_{\mu} N_{8}\right\}$. For the computation of the effective nucleon-nucleon interactions one should solve the field equations for $\Pi$ and $\tilde{V}_{\mu}$ as functionals of the baryon fields $N_{8}$ in bilinear order $\sim \bar{N}_{8} N_{8}$. The solution has to be reinserted into the effective action. As a result of this procedure one finds nucleon-nucleon interactions in the pseudoscalar channel and in the isospin-singlet vector channel mediated by the exchange of $V_{8 \mu}$ but not in the isospin-triplet vector channel. (The- $\sigma$ exchange term in the scalar channel is also contained in our model once the non-Goldstone scalar excitations in $\phi$ and $\chi$ are included.) This absence of isospin-triplet vector channel nucleon-nucleon interactions seems not to be consistent with observation 22].

In summary, three shortcomings indicate that the effective action (2.6) gives only an insufficient picture of the hadronic interactions in the vector channel: (i) the absence of a physical $S U(3)$-singlet vector state (the ninth vector meson), (ii) the inaccurate estimate of the parameter $a$ in eq. (7.6) for $\kappa_{f}=1$, (iii) the absence of nucleon-nucleon interactions in the isospin-triplet vector channel. In addition, no axial-vector mesons are present. These shortcomings can be overcome once we include the effective four-quark interactions in the color singlet vector and axial-vector channel. The successful relations (7.9), (7.17) and (7.18) can be maintained, whereas the parameter $a$ and the relation between $M_{\rho}, g_{\rho \pi \pi}$ and $f_{\pi}$ will be modified. Mixing effects with the divergence of the axial vector induce the correction (5.11) and therefore $\kappa_{f}<1$. 
The effects from vector and axial-vector four-quark interactions are discussed in detail in appendix A. Besides the "partial Higgs effect" (5.11) they account for the missing nucleon-nucleon interactions in the isospin triplet channel. They also contain the missing ninth vector meson, i.e. the $S U(3)_{C^{-}}$ singlet state, as well as the axial-vector mesons. Unfortunately, the additional effective interactions are parametrized by new unknown couplings. We will retain here only the "partial Higgs effect", the singlet vector meson $S^{\mu}$ with mass $\mu_{V}^{2}$ and the effective vector channel four-quark interaction. We omit the physics of axial-vector mesons. The relevant interactions discussed in appendix A lead then to an addition to the effective action (2.6) of the form $\left(S_{\mu \nu}=\partial_{\mu} S_{\nu}-\partial_{\nu} S_{\mu}\right)$

$$
\begin{aligned}
\mathcal{L}_{\rho}= & \frac{1}{4} S^{\mu \nu} S_{\mu \nu}+\frac{\mu_{V}^{2}}{2} S^{\mu} S_{\mu}+\frac{1}{\sqrt{6}} \tilde{c}_{\rho \bar{q} q} S^{\mu} \operatorname{Tr} \bar{N} \gamma_{\mu} N \\
& +\Delta \mathcal{L}_{k i n}^{(P)}+y_{N} \operatorname{Tr}\left\{\bar{N} \gamma_{\mu} \gamma^{5} \tilde{\hat{a}}^{\mu} N\right\}-\tau_{V} \operatorname{Tr}\left\{\bar{N} \gamma^{\mu} \lambda_{z} N\right\} \operatorname{Tr}\left\{\bar{N} \gamma_{\mu} \lambda_{z} N\right\}
\end{aligned}
$$

where the partial Higgs effects results in $\Delta \mathcal{L}_{k i n}^{(P)}$ as given by eq. (5.11) and the term $\sim y_{N}$ with

$$
\tilde{\hat{a}}_{\mu}=-\frac{i}{2}\left(\xi^{\dagger} D_{\mu} \xi-\xi D_{\mu} \xi^{\dagger}\right)-\frac{1}{3} \operatorname{Tr} a_{\mu}
$$

The couplings $\tilde{c}_{\rho \bar{q} q}, y_{N}$ and $\tau_{V}$ may be determined from phenomenology and we assume $\mu_{V}^{2} \approx \bar{M}_{\rho}^{2}$. There is actually no symmetry argument why $\mu_{V}^{2}$ should equal $\bar{M}_{\rho}^{2}=g^{2} \chi_{0}^{2}$. In particular, $\mu_{V}^{2}$ is not related to chiral and color symmetry breaking. We speculate that a partial fixed point in the renormalization flow of the ratio $\mu_{V}^{2} /\left(g^{2} \chi_{0}^{2}\right)$ could lead to an understanding of the puzzle of the phenomenologically required approximate "ninefold degeneracy" of the light vector meson masses.

All other effective interactions discussed in appendix A will be considered as subleading and neglected. Some of the couplings can be estimated from observations like the decay reates of axial-vector mesons into vector mesons and pseudoscalars. This may later be used for an estimate of the typical size of the neglected subleading terms.

\section{$9 \quad$ Interactions of pseudoscalar mesons}

The cubic interactions between the pseudoscalar and the baryon octets are usually parametrized by

$$
\mathcal{L}_{N}^{(p)}=F \operatorname{Tr}\left\{\bar{N}_{8} \gamma^{\mu} \gamma^{5}\left[\tilde{a}_{\mu}, N_{8}\right]\right\}+D \operatorname{Tr}\left\{\bar{N}_{8} \gamma^{\mu} \gamma^{5}\left\{\tilde{a}_{\mu}, N_{8}\right\}\right\}
$$

Experimental values are $F=0.459 \pm 0.008$ and $D=0.798 \pm 0.008$. The effective action (2.6) leads to (cf. eq. (5.22) $) F=D=0.5$. This can be considered as a good achievement since the reparametrization symmetry 
does not restrict the coupling constants $F$ and $D$. (This contrasts with the coupling of the vector current $\tilde{v}_{\mu}$.) In our context the relation $F=D=0.5$ is directly connected to the origin of these couplings from the quark kinetic term and therefore to quark-baryon duality. The term $\sim y_{N}$ from the partial Higgs effect (8.7) provides for a correction $F+D=1+y_{N}$ and one infers $y_{N} \approx 0.26$. Contributions to $D-F \approx 0.34$ have to be generated from other higher-order invariants, as, for example, a momentum-dependent Yukawa coupling involving $D_{\mu} \chi$. We note that $D-F$ contributes to the $\eta$-nucleon coupling but not to the interaction between protons, neutrons and pions. The latter can be written in a more conventional form with the nucleon doublet $\mathcal{N}^{T}=(p, n)$ and $\tilde{a}_{\mu}$ restricted to a $2 \times 2$ matrix (by omitting the last line and column)

$$
\begin{aligned}
\mathcal{L}_{\mathcal{N}}^{(\pi)} & =g_{A} \overline{\mathcal{N}} \gamma^{\mu} \gamma^{5} \tilde{a}_{\mu} \mathcal{N} \\
g_{A} & =F+D
\end{aligned}
$$

The inclusion of weak interactions will replace the derivative in the definition (5.5) of $\tilde{a}_{\mu}$ by a covariant derivative involving a coupling to the $W$-boson (see next section). The constant $g_{A}$ will therefore appear in the $\beta$-decay rate of the neutron.

For a discussion of the self-interactions of the pseudoscalar mesons we first expand eq. (5.24) in powers of $\Pi=\frac{1}{2} \Pi^{z} \lambda_{z}$

$$
\begin{aligned}
\mathcal{L}^{(\pi)} & =\frac{f^{2}}{4} \operatorname{Tr}\left\{\partial^{\mu} \exp \left(-\frac{2 i}{f} \Pi\right) \partial_{\mu} \exp \left(\frac{2 i}{f} \Pi\right)\right\} \\
& =\operatorname{Tr}\left\{\partial^{\mu} \Pi \partial_{\mu} \Pi\right\}+\frac{1}{f^{2}} \operatorname{Tr}\left\{\partial^{\mu} \Pi^{2} \partial_{\mu} \Pi^{2}-\frac{4}{3} \partial^{\mu} \Pi \partial_{\mu} \Pi^{3}\right\}+\ldots
\end{aligned}
$$

Similarly, the current quark mass term (5.28) contributes

$$
\begin{aligned}
\mathcal{L}_{j} & =\operatorname{Tr}\left\{M_{(p)}^{2} \Pi^{2}\right\}-\operatorname{Tr}\left\{\frac{M_{(p)}^{2}}{3 f^{2}} \Pi^{4}\right\}+\ldots \\
M_{(p)}^{2} & =2 Z_{\phi}^{-1 / 2} a_{q} \sigma_{0} f^{-2} \bar{m}
\end{aligned}
$$

This yields the low-momentum four-pion interactions. Further effective interactions arise from the exchange of vector mesons according to eq. (5.26). They are obtained by substituting for the vector mesons the solution of the field equation in presence of pseudoscalars

$$
\tilde{V}_{\mu}=-\frac{\bar{M}_{\rho}^{2}}{g} G_{\mu}^{(\rho) \nu} \tilde{v}_{\nu}+\ldots
$$

with the vector meson propagator $G^{(\rho)}$ obeying

$$
G_{\mu}^{(\rho) \nu}\left[\left(\bar{M}_{\rho}^{2}-\partial^{2}\right) \delta_{\nu}^{\sigma}+\partial_{\nu} \partial^{\sigma}\right]=\delta_{\mu}^{\sigma}
$$


One obtains

$$
\mathcal{L}_{V}=\chi_{0}^{2} \operatorname{Tr}\left\{\tilde{v}^{\mu}\left(\delta_{\mu}^{\sigma}-\bar{M}_{\rho}^{2} G_{\mu}^{(\rho) \sigma}\right) \tilde{v}_{\sigma}\right\}+\ldots
$$

and we note that the term $\chi_{0}^{2} \operatorname{Tr}\left\{\tilde{v}^{\mu} \tilde{v}_{\mu}\right\}$ in the expression (5.26) is cancelled by the lowest order of a derivative expansion of $G^{(\rho)}$. It should be remarked that $\operatorname{Tr}\left\{\tilde{v}^{\mu} \tilde{v}_{\mu}\right\}$ is invariant under the chiral transformations. It involves only two derivatives and cannot be reduced to the term $\operatorname{Tr}\left\{\partial^{\mu} U^{\dagger} \partial_{\mu} U\right\}$ which appears in chiral perturbation theory. It is, however, not consistent with the local reparametrization symmetry.

The next order in a derivative expansion of eq. (9.7) reads

$$
\mathcal{L}_{V}=\frac{1}{2 g^{2}} \operatorname{Tr}\left\{\tilde{v}^{\mu \nu} \tilde{v}_{\mu \nu}\right\}+\ldots
$$

where

$$
\tilde{v}_{\mu \nu}=\partial_{\mu} \tilde{v}_{\nu}-\partial_{\nu} \tilde{v}_{\mu}+i\left[\tilde{v}_{\mu}, \tilde{v}_{\nu}\right]
$$

has been completed to a covariant "field strength" such that (9.8) is invariant under the local reparametrization symmetry. With

$$
\tilde{v}_{\mu \nu}=-\frac{i}{4} \xi^{\dagger}\left(\partial_{\mu} U \partial_{\nu} U^{\dagger}-\partial_{\nu} U \partial_{\mu} U^{\dagger}\right) \xi
$$

one obtains

$$
\begin{aligned}
\mathcal{L}_{V}= & \frac{1}{16 g^{2}} \operatorname{Tr}\left\{\partial_{\mu} U^{\dagger} \partial^{\mu} U \partial_{\nu} U^{\dagger} \partial^{\nu} U-\partial_{\mu} U^{\dagger} \partial_{\nu} U \partial^{\mu} U^{\dagger} \partial^{\nu} U\right\} \\
= & \frac{1}{32 g^{2}}\left[6 \operatorname{Tr}\left\{\partial_{\mu} U^{\dagger} \partial^{\mu} U \partial_{\nu} U^{\dagger} \partial^{\nu} U\right\}-\left(\operatorname{Tr}\left\{\partial_{\mu} U^{\dagger} \partial^{\mu} U\right\}\right)^{2}\right. \\
& \left.-2 \operatorname{Tr}\left\{\partial_{\mu} U^{\dagger} \partial_{\nu} U\right\} \operatorname{Tr}\left\{\partial^{\mu} U^{\dagger} \partial^{\nu} U\right\}\right]
\end{aligned}
$$

One can infer the contribution of $\mathcal{L}_{V}$ to the parameters $L_{i}$ appearing in next to leading order in chiral perturbation theory [23]

$$
L_{1}^{(V)}=\frac{1}{32 g^{2}} \quad, \quad L_{2}^{(V)}=\frac{1}{16 g^{2}} \quad, \quad L_{3}^{(V)}=-\frac{3}{16 g^{2}}
$$

We observe that with the hypothesis of scalar Vervollständigung (2.6) the constants depend only on the effective gauge coupling $g$. For $g=6$ the values $L_{1}^{(V)}=0.87 \cdot 10^{-3}, L_{2}^{(V)}=1.74 \cdot 10^{-3}, L_{3}^{(V)}=-5.2 \cdot 10^{-3}$ compare well with the values [24] extracted from observation $L_{1}=(0.7 \pm 0.3) \cdot 10^{-3}, L_{2}=$ $(1.7 \pm 0.7) \cdot 10^{-3}, L_{3}=-(4.4 \pm 2.5) \cdot 10^{-3}$. This is consistent with the hypothesis that these constants are dominated ${ }^{27}$ by vector-meson exchange [26]. The momentum dependence of the effective four-pion vertex extracted from eq. (9.7) describes the fact the $\pi-\pi$ scattering at intermediate energies is dominated by the $\rho$-resonance. We conclude that the hypothesis of scalar Vervollständigung (2.6) gives a very satisfactory picture of the pion interactions.

\footnotetext{
${ }^{21}$ Further contributions arise from the exchange of scalars and have been estimated [25] as $L_{2}^{(S)}=0, L_{3}^{(S)}=1.3 \cdot 10^{-3}$.
} 


\section{Weak interactions}

Kaons decay into two pions by weak interactions. It is an old puzzle how the strong enhancement of the $\Delta I=1 / 2$ decays of $K_{S}^{0}$ as compared to the $\Delta I=3 / 2$ decay of $K^{ \pm}$should be explained in QCD. The semileptonic weak decays of kaons and pions are directly related to the corresponding decay constants $f_{K}, f_{\pi}$. These characteristic properties of the weak decays are described by our model once the weak interactions are incorporated. New parameters specify the strength of effective vertices which are one-particle irreducible with respect to cutting a $W^{ \pm}$-boson line. Following the philosophy underlying the effective action (2.6) we only include "renormalizable" interactions with dimension $\leq 4$. These invariants only contribute to the $\Delta I=1 / 2$ decays. Comparison with the $K_{S}^{0}$-decays determines the relevant parameter - ultimately it should be computed from QCD. The contribution of (one-particle reducible) $W$-boson exchange diagrams to the $\Delta I=3 / 2$ processes is substantially smaller. The corresponding effective vertex for $\phi$ and $\chi$ involves two more powers of these fields as compared to the leading invariant. Together with higher 1PI-vertices it should be considered as subleading. Scalar Vervollständigung leads to a qualitative understanding of the $\Delta I=1 / 2$ enhancement.

\section{a) Strangeness-violating local interactions}

The exchange of the heavy $W$ and $Z$ bosons in tree and loop diagrams produces multi-fermion interactions which may violate strangeness. On the momentum scale of interest here these interactions can be taken as local. After bosonisation, they result in two extensions of the effective action (2.6). All diagrams which decay into pieces by cutting a $W$ or $Z$ propagator can be represented by the couplings of a heavy boson to the fields present in the effective action (2.6). Those are retricted by the weak gauge symmetry. Keeping only the lowest dimension terms, we implement these couplings by appropriate covariant derivatives. The semileptonic decays are completely described by them. On the other hand, the diagrams which are one-particle irreducible with respect to cutting a $W$ or $Z$-boson line cannot be represented in this way. They result in additional local interactions which have to obey the appropriate symmetries.

First of all, the inclusion of weak interactions in our framework requires the effective action to be invariant under local $S U(2)_{L} \times U(1)_{Y}$ symmetry. On the other hand, the global flavor symmetry $S U(3)_{L} \times S U(3)_{R}$ is reduced to $S U(2)_{R} \times U(1)_{R}$. Here the global $S U(2)_{R}$ transformations act between right-handed down and strange quarks (which carry identical hypercharge) and $U(1)_{R}$ consists of phase rotations of the right-handed quarks with generator $\tilde{Q}$. In lowest order the strangeness-violating interactions obey, however, the larger $S U(3)_{R}$ flavor symmetry. (The latter is broken by the couplings of the $Z$-boson and the photon.) Also $C$ and $P$ need not be conserved separately any more, whereas $C P$ is an exact symmetry if effects from the third 
generation quarks are neglected. Of course, local $S U(2)_{L}$ symmetry requires the charmed quark. In this section we use an effective action where the heavy charmed quark has been integrated out.

Local $S U(2)_{L} \times U(1)_{Y}$ gauge symmetry is easily implemented by replacing all derivatives in the effective action (2.6) by appropriate covariant derivatives. Besides this we have to include the effects from additional local interactions which become possible due to the reduced flavor symmetry. They arise from effective short distance four-fermion interactions mediated by the exchange of $W^{ \pm}$or $Z^{0}$ in loops (i.e. "penguin diagrams") and therefore are suppressed by inverse powers of the squared $W$-boson mass $M_{W}^{2}$. As an example, a new local mass term for $\phi$ reads for a vanishing Cabibbo angle

$$
\mathcal{L}_{\tilde{\mu}}=\frac{g_{W}^{2} \tilde{\mu}_{W}^{4}}{4 M_{W}^{2}} \operatorname{Tr}\left\{\phi^{\dagger} \phi \lambda_{8}\right\}
$$

Here the weak gauge coupling $g_{W}$ and $M_{W}$ are related to the Fermi constant by $G_{F}=g_{W}^{2} /\left(4 \sqrt{2} M_{W}^{2}\right)$. The value of the parameter $\tilde{\mu}_{W}(k)$ can be computed for large $k$ by translating (10.1) into an effective four-quark interaction according to sect. 2 and comparing with the perturbatively computed value. For the present purpose we treat $\tilde{\mu}_{W} \equiv \tilde{\mu}_{W}(k=0)$ and similar constants as free parameters. The mass term (10.1) violates $C$ and $P$ and preserves $C P$. For a nonzero Cabibbo angle the field $\phi$ in (10.1) has to be replaced by the "weak interaction eigenstate" $\hat{\phi}$, which is related to the basis of mass eigenstates $\phi$ by

$$
\hat{\phi}=\phi R_{\theta}=\phi\left(\begin{array}{ccc}
1 & 0 & 0 \\
0 & c_{\theta} & -s_{\theta} \\
0 & s_{\theta} & c_{\theta}
\end{array}\right)
$$

Here $s_{\theta}$ and $c_{\theta}$ are the sine and cosine of the Cabibbo angle. The mass term (10.1) becomes

$$
\begin{aligned}
\mathcal{L}_{\tilde{\mu}} & =\frac{g_{W}^{2} \tilde{\mu}_{W}^{4}}{4 M_{W}^{2}} \operatorname{Tr}\left\{\phi^{\dagger} \phi \lambda_{W}\right\} \\
\lambda_{W} & =R_{\theta} \lambda_{8} R_{\theta}^{T}=\left(1-\frac{3 s_{\theta}^{2}}{2}\right) \lambda_{8}+\sqrt{3} c_{\theta} s_{\theta} \lambda_{6}+\frac{\sqrt{3}}{2} s_{\theta}^{2} \lambda_{3}
\end{aligned}
$$

and we underline the strangeness-violating contribution $\sim \lambda_{6}$. Inserting the nonlinear pseudoscalars (4.6) this term vanishes, however, and does not contribute to strangeness violation in the sector of the Goldstone bosons $\Pi$.

In leading order $M_{W}^{-2}$ the terms involving two derivatives of $\phi$ or $\chi$ are given by

$$
\mathcal{L}_{\mu}=\frac{g_{W}^{2} \mu_{W}^{2}}{4 M_{W}^{2}}\left[\operatorname{Tr}\left\{D^{\mu} \phi^{\dagger} D_{\mu} \phi \lambda_{W}\right\}+\delta_{W}\left(D^{\mu} \chi\right)_{i j, a b}^{*}\left(D_{\mu} \chi\right)_{i j, a c}\left(\lambda_{W}\right)_{c b}\right]
$$

The effective action (10.4) involves two additional real parameters $\mu_{W}^{2}$ and $\delta_{W}$. It is invariant under the local symmetry $S U(3)_{C} \times S U(2)_{L} \times U(1)_{Y}$, global 
$S U(3)_{R}$ flavor symmetry and $C P$, whereas it violates $P, C$ and strangeness. In lowest order in $M_{W}^{-2}$ and neglecting electromagnetic effects we may replace $D_{\mu} \phi$ by $\partial_{\mu} \phi$ and use (2.7) for $D_{\mu} \chi$. Furthermore, we will be interested only in strangeness-violating effects and replace $\lambda_{W}$ by $\sqrt{3} c_{\theta} s_{\theta} \lambda_{6}$. Concentrating on the pseudoscalar interactions we insert (5.5) and obtain

$$
\begin{aligned}
\mathcal{L}_{\mu U}= & \frac{1}{2} A_{W} g_{8} f^{2}\left[\operatorname{Tr}\left\{\partial^{\mu} U^{\dagger} \partial_{\mu} U \lambda_{6}\right\}+4 g_{\chi} \operatorname{Tr}\left\{\partial^{\mu} \xi^{\dagger} \partial_{\mu} \xi \lambda_{6}\right\}\right. \\
& \left.+\frac{4 i}{3} g_{\chi} \partial^{\mu} \theta \operatorname{Tr}\left\{\xi^{\dagger} \partial_{\mu} \xi \lambda_{6}\right\}\right]
\end{aligned}
$$

with

$$
\begin{aligned}
A_{W} & =\frac{c_{\theta} s_{\theta} g_{W}^{2} f^{2}}{4 M_{W}^{2}}=3.98 \cdot 10^{-8} \\
g_{8} & =\frac{\sqrt{3}}{2} \frac{\mu_{W}^{2}}{f^{2}} \frac{1-2 \delta_{W} x / 7}{1+x} \\
g_{\chi} & =\frac{9 \delta_{W} x}{14-4 \delta_{W} x}
\end{aligned}
$$

replacing $\mu_{W}$ and $\delta_{W}$. Adding the interactions with gluons leads to the replacements

$$
\begin{aligned}
i \xi^{\dagger} \partial_{\mu} \xi & \longrightarrow i\left(v_{\mu}+g \tilde{V}_{\mu}+a_{\mu}\right) \\
\partial^{\mu} \xi^{\dagger} \partial_{\mu} \xi & \longrightarrow\left(v^{\mu}+g \tilde{V}^{\mu}+a_{\mu}\right)\left(v_{\mu}+g \tilde{V}_{\mu}+a_{\mu}\right)
\end{aligned}
$$

This makes the local reparametrization invariance of $\mathcal{L}_{\mu U}$ manifest. We show in appendix B that only derivative terms contribute to strangeness-violating interactions for the pseudoscalars. One infers that $\mathcal{L}_{\mu}(10.4)$ is the only relevant bosonic term with dimension $\leq 4$. The invariant of dimension four for strangeness-violating fermionic interactions can be found in appendix B. This should dominate the hyperon decays.

\section{b) Vector-meson-W-boson mixing}

We also have to include the effects of local $S U(2)_{L} \times U(1)_{Y}$ symmetry in the derivative terms of eq. (2.6). The photon coupling has already been

discussed in sect. 3. The coupling of the weak gauge bosons $W_{\mu}^{ \pm}$, to the quarks is implemented by inserting in the effective action (2.6) the appropriate covariant derivatives

$$
\begin{aligned}
& \partial_{\mu} \psi_{L, R} \rightarrow \partial_{\mu} \psi_{L, R}+D_{\mu}^{(L, R)} \psi_{L, R} \\
& D_{\mu}^{(L)}=-\frac{i}{2} g_{W} \overrightarrow{\tilde{W}}_{\mu} \vec{\tau}_{W}=-i \frac{g_{W}}{\sqrt{2}}\left(\begin{array}{ccc}
0_{\mu}, & c_{\theta} \tilde{W}_{\mu}^{+}, & s_{\theta} \tilde{W}_{\mu}^{+} \\
c_{\theta} \tilde{W}_{\mu}^{-}, & 0, & 0 \\
s_{\theta} \tilde{W}_{\mu}^{-}, & 0, & 0
\end{array}\right) \\
& D_{\mu}^{(R)}=0
\end{aligned}
$$


We have omitted in the explicit form of $D_{\mu}^{(L, R)}$ the couplings of the $Z$-bosons since they do not contribute to strangeness-violating interactions $\left(\tau_{3 W}=\tau_{3}\right)$. They can easily be inserted in the formalism below. The weak coupling to the quark bilinears $\gamma_{i j} \sim \psi_{R i} \bar{\psi}_{L j}$ follows correspondingly[2 by replacing in eq. (2.6)

$$
D_{\mu} \gamma_{i j} \rightarrow D_{\mu} \gamma_{i j}+D_{\mu}^{(R)} \gamma_{i j}-\gamma_{i j} D_{\mu}^{(L)}
$$

Finally, we add the mass terms for the gauge bosons ${ }^{23}$

$$
\mathcal{L}_{W}=M_{W}^{2} \tilde{W}^{+\mu} \tilde{W}_{\mu}^{-}+\frac{1}{2} M_{Z}^{2} \tilde{Z}^{\mu} \tilde{Z}_{\mu}
$$

Eliminating the fields $W_{\mu}^{ \pm}, Z_{\mu}$ by solving their field equations as functionals of $\psi, \phi$ and $\chi$ yields effective four Fermi interactions ${ }^{24}$ relevant for weak interactions at low momenta as well as weak mesonic interactions. Furthermore, spontaneous color symmetry breaking by the octet expectation value $\langle\chi\rangle$ (2.18) leads to a mixing between $W, Z$-bosons and gluons similar to the photon mixing discussed in sect. 3. The mixing angle is tiny due to the large masses $M_{W}, M_{Z}$. It needs, however, to be included for strangeness-violating processes.

In the language of the nonlinear meson fields the weak interactions add to the effective Lagrangian a term $\mathcal{L}_{W}=\mathcal{L}_{W N}+\mathcal{L}_{W M}+\mathcal{L}_{W U}+\mathcal{L}_{\mu U}$

$$
\begin{aligned}
\mathcal{L}_{W N}= & i \operatorname{Tr}\left\{\bar{N}_{L} \gamma^{\mu} \xi D_{\mu}^{(L)} \xi^{\dagger} N_{L}+\bar{N}_{R} \gamma^{\mu} \xi^{\dagger} D_{\mu}^{(R)} \xi N_{R}\right\} \\
\mathcal{L}_{W M}= & M_{W}^{2} \tilde{W}^{+\mu} \tilde{W}_{\mu}^{-}+\frac{1}{2} M_{Z}^{2} \tilde{Z}^{\mu} \tilde{Z}_{\mu} \\
& -i g \chi_{0}^{2} \operatorname{Tr}\left\{\left(\xi^{\dagger} D_{\mu}^{(R)} \xi+\xi D_{\mu}^{(L)} \xi^{\dagger}\right) \tilde{V}^{\mu}\right\} \\
& -\left(\sigma_{0}^{2}+\frac{5}{9} \chi_{0}^{2}\right) \operatorname{Tr}\left\{D^{(L) \mu} D_{\mu}^{(L)}+D^{(R) \mu} D_{\mu}^{(R)}\right\} \\
& +\left(2 \sigma_{0}^{2}+\frac{1}{9} \chi_{0}^{2}\right) \operatorname{Tr}\left\{U^{\dagger} D^{(R) \mu} U D_{\mu}^{(L)}\right\}+\frac{1}{3} \chi_{0}^{2} \operatorname{Tr} D_{\mu}^{(R)} \operatorname{Tr} D_{\mu}^{(L)} \\
\mathcal{L}_{W U}= & \left(2 \sigma_{0}^{2}-\frac{1}{9} \chi_{0}^{2}\right) \operatorname{Tr}\left\{U^{\dagger} \partial^{\mu} U D_{\mu}^{(L)}+U \partial^{\mu} U^{\dagger} D_{\mu}^{(R)}\right\} \\
& +i \chi_{0}^{2} \operatorname{Tr}\left\{\left(\tilde{v}^{\mu}+\tilde{a}^{\mu}\right) D_{\mu}^{(L)}+\left(\tilde{v}^{\mu}-\tilde{a}^{\mu}\right) D_{\mu}^{(R)}\right\} \\
& -\frac{i}{3} \frac{\chi_{0}^{2}}{H_{\eta^{\prime}}} \partial^{\mu} \eta^{\prime} \operatorname{Tr}\left\{D_{\mu}^{(L)}-D_{\mu}^{(R)}\right\}
\end{aligned}
$$

with $\mathcal{L}_{\mu U}$ given above. These are the leading interactions for the strangenessviolating pseudoscalar meson decays. For other quantities like the $K_{L}^{0}-K_{S^{-}}^{0}$ mass difference they have to be supplemented by interactions discussed in appendix C.

\footnotetext{
${ }^{22}$ Covariant derivatives have also to be used in the additional interactions (8.7), (8.8).

${ }^{23}$ The kinetic terms for the weak gauge bosons and the Higgs sector are not important here.

${ }^{24}$ In the full standard model this also involves the leptons.
} 
For a computation of the mixing between $W_{\mu}^{ \pm}, \rho_{\mu}^{ \pm}$and $K_{\mu}^{* \pm}$ we can put $\xi=1$ and neglect the terms $\sim\left(D_{\mu}^{(L, R)}\right)^{2}$ in $\mathcal{L}_{W M}$. Inserting

$$
\tilde{V}_{\mu}=\frac{1}{\sqrt{2}}\left(\begin{array}{ccc}
0, & \tilde{\rho}_{\mu}^{+}, & \tilde{K}_{\mu}^{*+} \\
\tilde{\rho}_{\mu}, & 0, & 0 \\
\tilde{K}_{\mu}^{*-} & 0, & 0
\end{array}\right)
$$

and combining with the vector-meson mass term one obtains the squared mass matrix (with $\left.b=\left(\tilde{\rho}^{-}, \tilde{K}^{*-}, \tilde{W}^{-}\right), \mathcal{L}_{M}=b^{\dagger} \mathcal{M}_{b}^{2} b\right)$

$$
\begin{aligned}
\mathcal{M}_{b}^{2} & =\left(\begin{array}{ccc}
M_{\rho}^{2}, & 0, & B c_{\theta} \\
0, & M_{K^{*}}^{2}, & B s_{\theta} \\
B c_{\theta}, & B s_{\theta}, & M_{W}^{2}
\end{array}\right) \\
B & =-\frac{1}{2} g_{W} g \chi_{0}^{2}
\end{aligned}
$$

In a good approximation the fields $\left(\rho, K^{*}, W\right)$ for the physical spin-one bosons, i.e. the mass eigenstates, obey

$$
\begin{aligned}
\tilde{\rho}_{\mu} & =\rho_{\mu}-s_{\rho} W_{\mu}, \tilde{K}_{\mu}^{*}=K_{\mu}^{*}-s_{K} W_{\mu} \\
\tilde{W}_{\mu} & =W_{\mu}+s_{\rho} \rho_{\mu}+s_{K} K_{\mu}^{*}
\end{aligned}
$$

with

$$
s_{\rho}=\frac{1}{2} g_{W} g \frac{\chi_{0}^{2}}{M_{W}^{2}} c_{\theta}, s_{K}=\frac{1}{2} g_{W} g \frac{\chi_{0}^{2}}{M_{W}^{2}} s_{\theta}
$$

The mixing angles $s_{\rho}, s_{K}$ between $\tilde{\rho}, \tilde{K}^{*}$ and $\tilde{W}$ are suppressed by $B / M_{W}^{2}$ and therefore indeed tiny. The mass eigenvalues get only negligible corrections. Also the slight modification of the couplings of the physical $W$-boson (the mass eigenstate with mass $\approx M_{W}$ ) is of no importance. There remains, however, one relevant effect: The couplings of the physical $\rho^{ \pm}$and $K^{* \pm}$ mesons acquire from the mixing a small strangeness-violating correction. Indeed, expressed in terms of the "physical fields" one finds the contribution of charged spin-one bosons to $D_{\mu}^{(L)}$ (cf. eq. (10.8)

$D_{\mu}^{(L)}=-i \frac{g_{W}}{2 \sqrt{2}}\left[W_{\mu}^{+}+\frac{g_{W} g}{2} \frac{\chi_{0}^{2}}{M_{W}^{2}}\left(c_{\theta} \rho_{\mu}^{+}+s_{\theta} K_{\mu}^{*+}\right)\right]\left[c_{\theta}\left(\lambda_{1}+i \lambda_{2}\right)+s_{\theta}\left(\lambda_{4}+i \lambda_{5}\right)\right]+$ h.c.

Inserting this term in $\mathcal{L}_{M N}$, one sees that the $\rho$ meson couples not only to baryon-bilinears with zero strangeness but also has a small contribution of a coupling to $\bar{p} \Sigma$. It therefore contributes to strangeness violating weak decays. Similarly, the exchange of $\rho_{\mu}$ and $K_{\mu}^{*}$ induce additional strangeness violating interactions for the pseudoscalar mesons. We note that the mixing effects of the neutral weak boson $Z_{\mu}$ do not lead to violations of quantum numbers and can therefore be neglected. In summary, in leading order in $G_{F}$ the only relevant effect of the mixing between gluons and weak gauge bosons is the 
expression (10.18) for $D_{\mu}^{(L)}$. From $\mathcal{L}_{W M}$ we need then only to retain the piece $M_{W}^{2} W^{+\mu} W_{\mu}^{-}+\frac{1}{2} M_{Z}^{2} Z^{\mu} Z_{\mu}$.

\section{c) Leptonic meson decays}

Let us next turn to the leptonic decays of the pseudoscalar pions and kaons. The relevant vertex for the coupling of $W^{ \pm}$to the charged leptons $l$ and neutrinos $\nu_{l}$ is given by

$$
\begin{aligned}
\mathcal{L}_{l} & =\sum_{l} \frac{g_{W}}{2 \sqrt{2}}\left\{\bar{l} \gamma^{\mu}\left(1+\gamma^{5}\right) \nu_{l} \tilde{W}_{\mu}^{-}+\bar{\nu}_{l} \gamma^{\mu}\left(1+\gamma^{5}\right) l \tilde{W}_{\mu}^{+}\right\} \\
& =\sum_{l} \bar{\nu}_{l} \gamma^{\mu}\left(1+\gamma^{5}\right) l\left\{\frac{g_{W}}{2 \sqrt{2}} W_{\mu}^{+}+\frac{g_{W}^{2} g}{4 \sqrt{2}} \frac{\chi_{0}^{2}}{M_{W}^{2}}\left(c_{\theta} \rho_{\mu}^{+}+s_{\theta} K_{\mu}^{*+}\right)\right\}+\text { h.c. }
\end{aligned}
$$

The semileptonic pion decay can therefore in principle proceed via intermediate $W, \rho$ or $K^{*}$ exchange. In leading order in an expansion in $G_{F}$, however, only the $W$ exchange contributes. The relevant bilinear between $\Pi$ and $W$ obtains from

$$
\mathcal{L}_{W \Pi}=\frac{i}{2} f \partial^{\mu} \Pi^{z} \operatorname{Tr}\left\{\lambda_{z} D_{\mu}^{(L)}\right\}
$$

After eliminating $W$ by the field equation, one finds the effective cubic vertex

$$
\mathcal{L}_{\pi \mu \nu}=\frac{g_{W}^{2} c_{\theta} f}{4 \sqrt{2} M_{W}^{2}} \bar{\mu} \gamma^{\rho}\left(1+\gamma^{5}\right) \nu_{\mu} \partial_{\rho} \pi^{-}
$$

Comparison with the pion decay rate yields $f=f_{\pi}=92.5 \mathrm{MeV}$. In our approximation where $S U(3)$-violating vacuum expectation values are neglected we also have $f_{K}=f$. We have therefore taken in eq. (5.15) an average value. A detailed discussion of semileptonic decays including $S U(3)$-violating expectation values due to the nonvanishing mass of the strange quark can be found in ref. [15.

\section{d) Nonleptonic kaon decays}

For the non-leptonic weak decays of the kaons four effects need to be considered in linear order in $G_{F}$. The most important coupling arises from the one particle irreducible coupling (10.5). This is the only contribution in leading order. The second concerns the effective strangeness violating cubic vertices induced by the exchange of $\tilde{W}^{ \pm}$. A third contribution reflects the weak mixing between the pseudoscalars $\pi^{ \pm}, K^{ \pm}$and the scalars $a^{ \pm}, K^{* \pm}$. This last effect needs information about the effective potential $U(\gamma)$ and we will not discuss it here in a quantitative way. Finally, one expects "nonleading" strangeness violating vertices of the type (10.4) involving higher powers of $\phi, \chi$ or higher derivatives. A combination of these effects should explain the observed values

$$
\begin{aligned}
\Gamma\left(K_{S}^{0} \rightarrow \pi^{+} \pi^{-}\right) & =5.061 \cdot 10^{-12} \mathrm{MeV} \\
\Gamma\left(K_{S}^{0} \rightarrow \pi^{0} \pi^{0}\right) & =2.315 \cdot 10^{-12} \mathrm{MeV} \\
\Gamma\left(K^{-} \rightarrow \pi^{-} \pi^{0}\right) & =1.126 \cdot 10^{-14} \mathrm{MeV}
\end{aligned}
$$


which reflect the strong enhancement of the $\Delta I=1 / 2$ decays.

The cubic coupling for the pseudscalar octet arising from (10.5) needs some care. At first sight the terms $\sim g_{\chi}$ seem to contribute. These terms generate, however, also parity-violating mixings between the vector mesons $\tilde{v}_{\mu}$ and the pseudoscalars $a_{\mu} \sim \partial_{\mu} \Pi$ (cf. eq. (10.7)). Subsequent "decay" of intermediate vector bosons into two Goldstone bosons leads to contributions to the effective cubic vertex. This issue is most easily dealt with by "integrating out" the vector mesons, similar to sect. 9. In lowest order in the derivatives one has (cf. eq. (9.7)) $v_{\mu}+g \tilde{V}_{\mu}=0$. As a consequence one finds $\partial^{\mu} \xi^{\dagger} \partial_{\mu} \xi \rightarrow a^{\mu} a_{\mu}$ and concludes that the terms $\sim g_{\chi}$ do not contribute to the strangeness-violating cubic vertices. The effective cubic interaction therefore reads $\left(\partial^{2}=\partial^{\mu} \partial_{\mu}\right)$

$$
\begin{aligned}
\mathcal{L}_{\mu U}^{(3)}= & \frac{2 i}{f} A_{W} g_{8} \operatorname{Tr}\left\{\left[\partial^{\mu} \Pi \partial_{\mu} \Pi, \Pi\right] \lambda_{6}\right\} \\
= & \frac{1}{2 f} A_{W} g_{8}\left\{K _ { S } ^ { 0 } \left[6 \partial^{\mu} \pi^{+} \partial_{\mu} \pi^{-}+3 \partial^{\mu} \pi^{0} \partial_{\mu} \pi^{0}\right.\right. \\
& \left.+\pi^{+} \partial^{2} \pi^{-}+\pi^{-} \partial^{2} \pi^{+}+\pi^{0} \partial^{2} \pi^{0}\right]+i K_{L}^{0}\left[\pi^{-} \partial^{2} \pi^{+}-\pi^{+} \partial^{2} \pi^{-}\right] \\
& \left.-i \pi^{0}\left[K^{+} \partial^{2} \pi^{-}-K^{-} \partial^{2} \pi^{+}\right]+i \partial^{2} \pi^{0}\left[K^{+} \pi^{-}-K^{-} \pi^{+}\right]\right\}
\end{aligned}
$$

Here we have only retained terms involving one kaon and two pions in the second expression and we use

$$
K_{S}^{0}=\frac{i}{\sqrt{2}}\left(K^{0}-\bar{K}^{0}\right)=\Pi^{7} \quad, \quad K_{L}^{0}=\frac{1}{\sqrt{2}}\left(K^{0}+\bar{K}^{0}\right)=\Pi^{6}
$$

Evaluated on mass-shell $\left(\partial^{2} \rightarrow M^{2}\right)$, this vertex reduces to

$$
\begin{aligned}
\mathcal{L}_{\mu U}^{(3)}= & -\frac{1}{2 f} A_{W} g_{8}\left\{\left(3 M_{K}^{2}-4 M_{\pi}^{2}\right) K_{S}^{0} \pi^{+} \pi^{-}\right. \\
& \left.+\left(\frac{3}{2} M_{K}^{2}-2 M_{\pi}^{2}\right) K_{S}^{0} \pi^{0} \pi^{0}+i\left(M_{\pi^{+}}^{2}-M_{\pi^{0}}^{2}\right)\left(K^{-} \pi^{+} \pi^{0}-K^{+} \pi^{-} \pi^{0}\right)\right\}
\end{aligned}
$$

Up to tiny isospin-violating corrections from the difference between charged an neutral pion masses it only contributes to the decay $K_{S}^{0} \rightarrow 2 \pi$. With

$$
\begin{aligned}
\Gamma^{(3)}\left(K_{S}^{0} \rightarrow 2 \pi^{0}\right)=\Gamma^{(3)}\left(K_{S}^{0} \rightarrow \pi^{+} \pi^{-}\right)= \\
-\frac{A_{W}}{2 f} g_{8}\left(3 M_{K}^{2}-4 M_{\pi}^{2}\right)=-1.26 \cdot 10^{-4} g_{8} \mathrm{MeV}
\end{aligned}
$$

one obtains the partial width for $K_{S}^{0} \rightarrow 2 \pi^{0}$

$$
\Gamma\left(K_{S}^{0} \rightarrow 2 \pi^{0}\right)=2.66 \cdot 10^{-13} g_{8}^{2} \mathrm{MeV}
$$

Comparison with the experimental value requires

$$
g_{8}=2.95
$$


This corresponds to a very reasonable value $\mu_{W} \approx 440 \mathrm{MeV}$. From eq. (10.26) one also infers that the decay rate $\Gamma\left(K_{S}^{0} \rightarrow \pi^{+} \pi^{-}\right)$is twice the one for the decay into neutral pions, in accordance with observation (see below eq. (10.41) for a correction to this relation). The vertex (10.5) does not contribute to the charged kaon decay. The latter will only be induced by $\tilde{W}$ exchange, pseudoscalar-scalar mixing and nonleading 1PI vertices. We will see below that these effects are suppressed compared to the vertex (10.25). If a computation of the effective coupling $g_{8}$ from the standard model couplings yields indeed the value $(10.28)$ the dominance of the $\Delta I=1 / 2$ kaon decays can be naturally understood.

In order to compute the contribution of $\tilde{W}$-exchange to the effective strangeness-violating cubic vertex we need the strangeness-violating twopoint functions (10.20) $\left(\Pi=\frac{1}{2} \Pi^{z} \lambda_{z}\right)$

$$
\mathcal{L}_{W \Pi}=\frac{g_{W} f}{\sqrt{2}} \operatorname{Tr}\left\{\partial^{\mu} \Pi \hat{\lambda}_{+}\right\}\left(W_{\mu}^{+}+s_{\rho} \rho_{\mu}^{+}+s_{K} K_{\mu}^{*+}\right)+\text { h.c. }
$$

and the cubic vertex

$$
\begin{aligned}
\mathcal{L}_{W \Pi \Pi} & =\frac{1+5 x / 14}{1+x} \operatorname{Tr}\left\{\left[\Pi, \partial^{\mu} \Pi\right] D_{\mu}^{(L)}\right\} \\
& =-\frac{i g_{W}}{\sqrt{2}} \frac{1+5 x / 14}{1+x} \operatorname{Tr}\left\{\left[\Pi, \partial^{\mu} \Pi\right] \hat{\lambda}_{+}\right\} W_{\mu}^{+}+\text {h.c. }+\ldots
\end{aligned}
$$

with

$$
\hat{\lambda}_{+}=\frac{1}{2} c_{\theta}\left(\lambda_{1}+i \lambda_{2}\right)+\frac{1}{2} s_{\theta}\left(\lambda_{4}+i \lambda_{5}\right)
$$

Consider first the contribution from $W$-exchange. Inserting the solution of the field equation

$$
W_{\mu}^{+}=-\frac{g_{W}}{\sqrt{2} M_{W}^{2}} \operatorname{Tr}\left\{\left(f \partial_{\mu} \Pi-i \frac{1+5 x / 14}{1+x}\left[\Pi, \partial_{\mu} \Pi\right]\right) \hat{\lambda}_{+}^{\dagger}\right\}
$$

into $(10.29),(10.30),(10.12)$, one finds

$$
\begin{aligned}
\mathcal{L}_{\Delta S}^{(W)}= & i \frac{g_{W}^{2} f}{2 M_{W}^{2}} \frac{1+5 x / 14}{1+x} \hat{\mathcal{L}}_{\Delta S}, \\
\hat{\mathcal{L}}_{\Delta S}= & \operatorname{Tr}\left\{\partial^{\mu} \Pi \hat{\lambda}_{+}\right\} \operatorname{Tr}\left\{\left[\Pi, \partial_{\mu} \Pi\right] \hat{\lambda}_{+}^{\dagger}\right\}+\operatorname{Tr}\left\{\partial^{\mu} \Pi \hat{\lambda}_{+}^{\dagger}\right\} \operatorname{Tr}\left\{\left[\Pi, \partial_{\mu} \Pi\right] \hat{\lambda}_{+}\right\} \\
= & -\frac{1}{4} c_{\theta} s_{\theta}\left\{2 K^{+} \partial^{\mu} \pi^{-} \partial_{\mu} \pi^{0}+2 K^{+} \pi^{-} \partial^{\mu} \partial_{\mu} \pi^{0}-K^{+} \partial^{\mu} \partial_{\mu} \pi^{-} \pi^{0}\right. \\
& \left.+2 \sqrt{2} K^{0} \partial^{\mu} \pi^{-} \partial_{\mu} \pi^{+}+\sqrt{2} K^{0} \partial^{\mu} \partial_{\mu} \pi^{-} \pi^{+}\right\}- \text {c.c. }
\end{aligned}
$$

In the last expression we again have only listed the vertices involving one kaon and two pions. Another contribution arises from the exchange of $\rho$ and

\footnotetext{
${ }^{25}$ For $\left|\delta_{W} x\right| \ll 7 / 2$ the contribution $\sim \delta_{W}$ is small. We have used $x=4, \delta_{W}=0$ for the numerical value. Negative $\delta_{W}$ lead to smaller values of $\mu_{W}$.
} 
$K^{*}$ mesons due to the strangeness-violating mixing (10.29). It is computed similarly by inserting

$$
\begin{aligned}
\tilde{V}_{\mu}^{\Delta S}=- & \frac{g_{W} f}{4}\left[s_{\rho}\left(M_{\rho}^{2}-\partial^{2}\right)^{-1}\left(\lambda_{1}+i \lambda_{2}\right)+s_{K}\left(M_{K^{*}}^{2}-\partial^{2}\right)^{-1}\left(\lambda_{4}+i \lambda_{5}\right)\right] . \\
& \operatorname{Tr}\left\{\partial^{\mu} \Pi \hat{\lambda}_{+}^{\dagger}\right\}+\text { c.c. }
\end{aligned}
$$

into eqs. (8.1), (10.29). One finds

$$
\begin{aligned}
\mathcal{L}_{\Delta S}^{\rho, K^{*}}= & i \frac{g_{W}^{2} g^{2} \chi_{0}^{4}}{4 f M_{W}^{2}} \operatorname{Tr}\left\{[ \Pi , \partial _ { \mu } \Pi ] \left[c_{\theta}\left(M_{\rho}^{2}-\partial^{2}\right)^{-1} \frac{\lambda_{1}+i \lambda_{2}}{2}\right.\right. \\
& \left.\left.+s_{\theta}\left(M_{K^{*}}^{2}-\partial^{2}\right)^{-1} \frac{\lambda_{4}+i \lambda_{5}}{2}\right]\right\} \operatorname{Tr}\left\{\partial^{\mu} \Pi \hat{\lambda}_{+}^{\dagger}\right\}+\text { c.c }
\end{aligned}
$$

We observe that in the approximation $M_{\rho}^{2}-\partial^{2}=M_{K^{*}}^{2}-\partial^{2}=\bar{M}_{\rho}^{2}$ the cubic vertex (10.35) is proportional to (10.33), with a relative factor $\frac{9 x}{14+5 x}$. In this limit the total strangeness-violating contribution to the cubic vertex from $W, \rho$ and $K^{*}$ exchange

$$
\mathcal{L}_{\Delta S}^{\tilde{W}}=\mathcal{L}_{\Delta S}^{W}+\mathcal{L}_{\Delta S}^{\rho, K^{*}}=i \frac{g_{W}^{2} f}{2 M_{W}^{2}} \hat{\mathcal{L}}_{\Delta S}
$$

does not depend 0 on $x$. From eq. (10.36) we can extract the contributions to the on-shell cubic vertices

$$
\begin{aligned}
i \Gamma_{(\tilde{W})}^{(3)}\left(K^{+} \rightarrow \pi^{+} \pi^{0}\right) & =-\frac{1}{2} \Gamma_{(\tilde{W})}^{(3)}\left(K_{S}^{0} \rightarrow \pi^{+} \pi^{-}\right)=\frac{g_{W}^{2} f}{8 M_{W}^{2}} c_{\theta} s_{\theta}\left(M_{K}^{2}-M_{\pi}^{2}\right) \\
& =4.2 \cdot 10^{-5} \mathrm{MeV} \\
\Gamma_{(\tilde{W})}^{(3)}\left(K_{S}^{0} \rightarrow \pi^{0} \pi^{0}\right) & =0
\end{aligned}
$$

We finally have to inlcude the contributions from scalar pseudoscalar mixing and from nonleading 1PI vertices. As discussed in appendix B, the interesting contribution can be parametrized as

$$
\Delta \mathcal{L}_{\Delta S}=-i \frac{g_{W}^{2} f}{2 M_{W}^{2}} z_{W} \hat{\mathcal{L}}_{\Delta S}
$$

with $z_{W}$ of order one. In consequence, this leads to the multiplication of the $W$-exchange contribution (10.36) by a factor $\left(1-z_{W}\right)$.

The partial decay width for $K \rightarrow \pi \pi$ is given by

$$
\begin{aligned}
\Gamma(K \rightarrow \pi \pi) & =F_{K \pi \pi}\left|\Gamma^{(3)}(K \rightarrow \pi \pi)\right|^{2} \\
F_{K \pi \pi} & =\frac{1}{8 \pi} \frac{\left|\vec{p}_{\pi}\right|}{M_{K^{2}}}=3.347 \cdot 10^{-5} \mathrm{MeV}^{-1}
\end{aligned}
$$

\footnotetext{
${ }^{26}$ Alternatively, the effective interactions $\mathcal{L}_{\Delta S}^{\tilde{W}}$ can be evaluated by first integrating out the vector bosons $\tilde{V}_{\mu}$. The leading term follows from inserting convariant derivatives in $\mathcal{L}_{U}(5.24)$ and then integrating out the $\tilde{W}$-bosons.
} 
with an additional factor $1 / 2$ for two identical $\pi^{0}$. One finds

$$
\Gamma\left(K^{-} \rightarrow \pi^{-} \pi^{0}\right)=5.92 \cdot 10^{-14}\left(1-z_{W}\right)^{2} \mathrm{MeV}
$$

For the reasonable value $z_{W}=0.56$ this is compatible with observation. The same parameter $z_{W}$ also determines the ratio (for $\mu_{W}^{2}>0$ )

$$
\frac{\Gamma\left(K_{S}^{0} \rightarrow \pi^{+} \pi^{-}\right)}{\Gamma\left(K_{S}^{0} \rightarrow 2 \pi^{0}\right)}=2\left(1+\frac{0.84\left(1-z_{W}\right)}{3.71}\right)^{2}=2.2
$$

This is in perfect agreement with the observed value 2.19, reflecting the fact that the kaon decays are governed by only two independent amplitudes.

The overall scales appearing in the kaon decays can be visualized easily from the effective vertex which obtains from "integrating out" the oneparticle reducible $W$-boson exchange

$$
\mathcal{L}_{\Delta S}^{\tilde{W}}=\frac{g_{W}^{2}}{8 M_{W}^{2}} \operatorname{Tr}\left\{\left(\phi^{\dagger} \partial_{\mu} \phi-\partial_{\mu} \phi^{\dagger} \phi\right) \vec{\tau}_{W}\right\} \operatorname{Tr}\left\{\left(\phi^{\dagger} \partial^{\mu} \phi-\partial^{\mu} \phi^{\dagger} \phi\right) \vec{\tau}_{W}\right\}+\ldots
$$

where the dots stand for terms involving $\chi$. Comparison with the leading invariant (10.4) shows that essentially $\mu_{W}^{2}$ is replaced by two powers of $\sigma_{0}$ or $\chi_{0}$. The resulting relative suppression factor $f^{2} / \mu_{W}^{2}$ accounts for most of the relative suppression of the $\Delta I=3 / 2$ amplitude. A smaller additional suppression arises from the partial cancellation $1-z_{W}=0.44$. We observe that the suppression is not visible on the level of chiral perturbation theory. The invariant relevant for the $\Delta I=3 / 2$ decays

$$
\mathcal{L}_{\Delta I=3 / 2}=\left(1-z_{W}\right) \mathcal{L}_{\Delta S}^{\tilde{W}}=\frac{\left(1-z_{W}\right) g_{W}^{2} f^{4}}{32 M_{W}^{2}} \operatorname{Tr}\left\{U^{\dagger} \partial^{\mu} U \vec{\tau}_{W}\right\} \operatorname{Tr}\left\{U^{\dagger} \partial_{\mu} U^{\dagger} \vec{\tau}_{W}\right\}
$$

contains two derivatives just as the one (10.5) relevant for the $\Delta I=1 / 2$ decays.

In summary, the $\Delta I=1 / 2$ enhancement of the hadronic kaon decays can be qualitatively explained by two ingredients. Since for the pseudoscalar interactions only derivative terms are relevant, the leading dimensionless operator consistent with the symmetries is quadratic in $\phi$ or $\chi$. On the quark level it corresponds to diagrams involving two left-handed quarks. These penguin-type diagrams contribute only to $\Delta I=1 / 2$ decays. On the other hand, the diagrams contributing to $\Delta I=3 / 2$ decays involve at least four left-handed quarks. On the mesonic level they correspond to effective operators involving at least four powers of $\phi$ or $\chi$. Such higher dimension operators are suppressed according to the hypothesis of scalar Vervollständigung. Operators of this type can arise from one particle reducible $W$-boson exchange or from box-type diagrams where the $W$-boson exchange is supplemented by gluon exchange. An estimate of the direct $W$-exchange does not involve unknown parameters and shows indeed a small $\Delta I=3 / 2$ amplitude. The 
box-type 1PI diagrams have the same structure as the $W$-exchange contribution. Their negative relative sign is related to the sign of the appropriate anomalous dimension of the relevant four-quark operator. The corresponding factor $\left(1-z_{W}\right)$ leads to a partial cancellation of the $\Delta I=3 / 2$ amplitude. This further reduces the decay width for the hadronic decays of the charged kaons. The small parameter appearing in the relative suppression of the $\Delta i=3 / 2$ amplitude is the size of chiral symmetry breaking $\sim f$ divided by a typical hadronic scale $\sim \mu_{W}$.

\section{Diquark condensates}

At high baryon density one expects quark pairs to condensate [9]. In particular, the color-flavor locking [10] at high density ressembles in several aspects the spontaneous color symmetry breaking in the vacuum discussed in the preceeding sections. In this section we reformulate the diquark condensation in terms of effectice bosonic diquark fields $\Delta \sim \psi \psi$. In contrast to the color octet $\chi \sim \bar{\psi} \psi$ the diquark fields carry nonzero baryon number. If diquark condensation occurs at high density, the global symmetry corresponding to baryon number is spontaneously broken. There is therefore an order parameter which distinguishes the high density phase with $\langle\Delta\rangle \neq 0$ from the low density phase with $\langle\Delta\rangle=0$. This implies the existence of a true phase transition.

In addition to the fields discussed previously we consider in this section scalar fields with the transformation properties of quark-quark pairs. In our notation, these additional scalar fields are represented by complex $3 \times 3$ matrices $\Delta_{L}, \Delta_{R}$ obeying

$$
\delta \Delta_{L}=-i \Theta_{C}^{T} \Delta_{L}-i \Delta_{L} \Theta_{L} \quad, \quad \delta \Delta_{R}=-i \Theta_{C}^{T} \Delta_{R}-i \Delta_{R} \Theta_{R}
$$

The "quark pairs" $\Delta_{L(R)} \sim \psi_{L(R)} \psi_{L(R)}$ belong to a color antitriplet and carry baryon number $2 / 3$. The discrete symmetries act as $P: \Delta_{L} \leftrightarrow \Delta_{R}, \quad C$ : $\Delta_{L} \leftrightarrow \Delta_{R}^{*}$. For an appropriate scaling of the fields, the most general effective Lagrangian consistent with these symmetries and containing operators up to dimension four is

$$
\begin{aligned}
\mathcal{L}_{\Delta}= & \operatorname{Tr}\left\{\left(\partial^{\mu} \Delta_{L}^{\dagger}-i g \Delta_{L}^{\dagger} A_{\mu}^{*}\right)\left(\partial_{\mu} \Delta_{L}+i g A_{\mu}^{T} \Delta_{L}\right)\right. \\
& \quad+\left(\partial^{\mu} \Delta_{R}^{\dagger}-i g \Delta_{R}^{\dagger} A_{\mu}^{*}\right)\left(\partial_{\mu} \Delta_{R}+i g A_{\mu}^{T} \Delta_{R}\right\} \\
& +U_{\Delta}\left(\phi, \chi, \Delta_{L}, \Delta_{R}\right) \\
+ & +\left[h_{\Delta} \operatorname{Tr}\left\{\Delta_{L}^{\dagger} \tilde{\delta}_{L}+\Delta_{R}^{\dagger} \tilde{\delta}_{R}\right\}+\text { c.c }\right]
\end{aligned}
$$

Here the quark-quark bilinears $\left(\tilde{\delta}_{L}\right)_{i a},\left(\tilde{\delta}_{R}\right)_{i a}$ are defined as

$$
\left(\tilde{\delta}_{L, R}\right)_{i a}=\left(\psi_{L, R}\right)_{b j \beta} c^{\beta \gamma}\left(\psi_{L, R}\right)_{c k \gamma} \epsilon_{i j k} \epsilon_{a b c}
$$


They transform under all continuous symmetries precisely like (bosonic) antiquarks $\left(\psi_{L}^{\dagger}\right)_{i a},\left(\psi_{R}^{\dagger}\right)_{i a}$. (Here $\beta, \gamma=1,2$ are (Weyl-)spinor indices and $c^{\beta \gamma}=\epsilon^{\beta \gamma}$ is the appropriate charge conjugation matrix. Our conventions [11] are chosen such that parity transforms $\tilde{\delta}_{L} \leftrightarrow \tilde{\delta}_{R}$.) One observes that $\Delta_{L}$ transforms as $\delta_{L}$ and similarly for $\Delta_{R}$ and $\delta_{R}$. The addition to the effective potential

$$
\begin{aligned}
U_{\Delta}= & V_{\Delta}\left(\Delta_{L}, \Delta_{R}\right)+\gamma_{\phi} \operatorname{Tr}\left(\phi^{\dagger} \Delta_{R}^{\dagger} \Delta_{L}+\phi \Delta_{L}^{\dagger} \Delta_{R}\right) \\
& +\gamma_{\chi}\left[\left(\Delta_{R}\right)_{i a} \chi_{i j, a b}\left(\Delta_{L}^{\dagger}\right)_{b j}+\left(\Delta_{L}\right)_{i a} \chi_{j i b a}^{*}\left(\Delta_{R}^{\dagger}\right)_{b j}\right] \\
& +\epsilon\left[\left(\Delta_{L} \phi^{\dagger}\right)_{i a} \chi_{i j, a b}\left(\Delta_{L}^{\dagger}\right)_{b j}+\left(\Delta_{R} \phi\right)_{i a} \chi_{j i b a}^{*}\left(\Delta_{R}^{\dagger}\right)_{b j}\right. \\
& \left.+\left(\Delta_{L}\right)_{i a} \chi_{j i b a}^{*}\left(\phi \Delta_{L}^{\dagger}\right)_{b j}+\left(\Delta_{R}\right)_{i a} \chi_{i j a b}\left(\phi^{\dagger} \Delta_{R}^{\dagger}\right)_{b j}\right] \\
& +\ldots
\end{aligned}
$$

involves real parameters $\gamma_{\phi}, \gamma_{\chi}$, and $\epsilon$. The dots stand for terms involving $\Delta, \Delta^{\dagger}$ and two powers of $\phi$ or two powers of $\chi$. The additional potential $U_{\Delta}$ conserves the axial $U(1)$ symmetry. Electromagnetic and weak interactions are implemented by inserting the appropriate covariant derivatives.

In order to visualize the physical content in this regime, it is convenient to use nonlinear coordinates in field space, given by hermitean matrices $D_{L}, D_{R}$,

$$
\Delta_{L}=v^{\dagger} D_{L} W_{L}^{\dagger}, \quad \Delta_{R}=v^{\dagger} D_{R} W_{R}^{\dagger}
$$

Both $D_{L}$ and $D_{R}$ are color singlets with the transformation properties

$$
\delta D_{L}=i\left[\Theta_{P}, D_{L}\right], \quad \delta D_{R}=i\left[\Theta_{P}, D_{R}\right]
$$

With respect to the local reparametrization symmetry and the physical global $S U(3)$-symmetry $D_{L}$ and $D_{R}$ transform as singlets plus octets. A possible expectation value of the singlets (proportional to the unit matrix)

$$
<D_{L}>=<D_{R}>=\delta_{0}
$$

also preserves parity and charge conjugation. For $\delta_{0} \neq 0$ baryon number is, however, spontaneously broken. From

$$
\delta_{e m} \Delta_{L, R}=-i \beta \Delta_{L, R} \tilde{Q} \quad, \quad \delta_{e m} D_{L, R}=i \beta\left[\tilde{Q}, D_{L, R}\right]
$$

one infers that the electromagnetic $U(1)$-symmetry is modified in the Higgs picture (similar to the octet condensate) whereas the electric charge of $\delta_{0}$ is zero.

The diquarks $\Delta_{L, R}$ carry baryon number $\tilde{B}=2 / 3$. From

$$
\Delta_{L}^{\dagger} \Delta_{L}=W_{L} D_{L}^{2} W_{L}^{\dagger}, \Delta_{R}^{\dagger} \Delta_{R}=W_{R} D_{R}^{2} W_{R}^{\dagger}
$$

and the fact that $W_{L, R}$ have $\tilde{B}=0$, one concludes that $D_{L}$ and $D_{R}$ cannot carry nonzero $\tilde{B}$. The definition of the nonlinear fields (11.5) implies then 
directly that $v^{\dagger}$ must have $\tilde{B}=2 / 3$ in accordance with the discussion in sect. 6. In fact, up to reparametrization transformations the decomposition of $\phi, \Delta_{L}$ and $\Delta_{R}$ into the nonlinear fields is unique, except of special points in field space where some fields are not invertible or degenerate. For $D_{L}=$ $D_{R}=\delta, \delta \neq 0$ the association of $v^{\dagger}=\Delta_{L} W_{L} / \delta$ with a diquark field is particularly apparent.

For small $\Delta_{L}=\Delta_{R}=\delta=\delta^{*}$ one may expand $V_{\Delta}=m_{\Delta}^{2} \operatorname{Tr}\left\{\Delta_{L}^{\dagger} \Delta_{L}+\right.$ $\left.\Delta_{R}^{\dagger} \Delta_{R}\right\}$ such that $U_{\Delta}=\frac{1}{2} M_{\delta}^{2} \delta^{2}+\ldots$ with

$$
M_{\delta}^{2}=12 m_{\Delta}^{2}+12 \gamma_{\phi} \sigma_{0}+\frac{32}{\sqrt{6}} \gamma_{\chi} \chi_{0}\left(1+2 \epsilon \sigma_{0}\right)+\ldots
$$

Typically, the masses and couplings will depend on the baryon chemical potential $\mu_{B}$. Baryon number conservation in the vacuum requires $M_{\delta}^{2}>0$ for $\mu_{B}=0$. In the spirit of the discussion in sect. 2 the couplings also depend on the renormalization scale $k$. In particular, for large $k$ (and $\mu_{B}=0$ ) perturbative QCD should lead to a positive $m_{\Delta}$ and one can neglect $\sigma_{0}$ and $\chi_{0}$. In presence of quarks the field equation for $\Delta_{L, R}$ becomes then (for $\left.q^{2} \ll m_{\Delta}^{2}\right)$

$$
\Delta_{L, R}=-\frac{h_{\Delta}}{m_{\Delta}^{2}} \tilde{\delta}_{L, R}
$$

This shows the connection between the composite fields $\Delta_{L, R}$ and quarkquark bilinears.

For $k=0$ and large baryon density (large $\mu_{B}$ ) one expects the minimum of $U_{\Delta}$ to occur for $\delta_{0} \neq 0$. The particle content in the sectors of pseudoscalars, vector mesons and fermions may again be obtained from a non-linear representation by setting $D_{L}=D_{R}=\delta_{0}$ in eq. (11.5). As before, the field $v$ disappears from the effective action and only color singlets for possible physical excitations remain. Inserting eq. (11.5) into eq. (11.2) leads to expressions that are similar to sects. 4,5 in many respects. This is not surprising - except for the spontaneous breaking of baryon symmetry the symmetries are the same.

At this place we note that for $\delta_{0} \neq 0$ there are terms in the effective potential linear in $\chi$. They arise from the cubic term $\sim \gamma_{\chi}$ or the quartic term $\sim \epsilon$ in eq. (11.4). As a consequence, no solution $\chi_{0}=0$ is possible for $\delta_{0} \neq 0$ - the diquark condensation necessarily induces a color octet $<\bar{\psi} \psi>$ condensate! (In the limit where the nonlinear part of the effective potential for $\chi_{0}$ is dominated by a quadratic term $\sim \frac{1}{2} \mu_{\chi}^{2}\left(\sigma_{0}, \delta_{0}\right) \chi_{0}^{2}$, the expectation value is given by $\chi_{0}=-16 \gamma_{\chi}\left(1+2 \epsilon \sigma_{0}\right) \delta_{0}^{2} /\left(\sqrt{6} \mu_{\chi}^{2}\right)$.)

In our scenario the octet condensate $\chi_{0}$ is therefore different from zero both for small and large baryon density. The only qualitative change at high density is the spontaneous breaking of baryon number by $\delta_{0} \neq 0$. A secondor first-order phase transition line separates the low density phase with $\delta_{0}=0$ from the "quark matter" phase at high density. Due to the spontaneously 
broken abelian baryon number symmetry quark matter is a superfluid [10]. If the phase transition is of second order it belongs to the universality class of the $O(2)$-Heisenberg model. It is plausible that the transition line to quark matter could bifurcate from the line of first order transitions between a nucleon gas and nuclear matter (nucleon liquid). (This latter transition line ends in an endpoint with critical Ising behavior.) If so, there is no distinction between nuclear matter and quark matter at low enough temperature. For $T=0$ both nuclear and quark matter would be superfluids with spontaneously broken baryon number. They should be identified thus leading to a further manifestation of quark-baryon duality.

\section{Heavy quarks}

In this short section we extend our description to the heavy quarks carrying quantum numbers of charm, beauty and topness. These quarks are neutral under the global $S U(3)_{L} \times S U(3)_{R}$ symmetry. With respect to the physical $S U(3)_{V}$ symmetry they therefore transform as antitriplets. The physical electric charge has a contribution from $Q_{c}$ similar to table 1 . In consequence, the charmed fermions carry electric charge $0,1,1$. In complete analogy with the discussion in sect. 6 they also carry integer baryon number. We identify them with the charmed baryon antitriplet $\Xi_{c}^{0}(2472 \mathrm{MeV}, d s c), \Xi_{c}^{+}(2466 \mathrm{MeV}$, $u s c)$ and $\Lambda_{c}^{+}(2284 \mathrm{MeV}, u d c)$ where we have given the mass and the standard quark content in brackets. In the nonlinear language the charmed baryons consist of the $c$-quark and the nonlinear diquark field $v^{\dagger}$ which carries the quantum numbers of two light quarks. Similarly, the fermions with beauty carry electric charge $-1,0,0$. They are identified with $(d s b),(u s b)$ and $\Lambda_{b}^{0}$ $(5641 \mathrm{MeV}, u d b)$. The quantum numbers of the fermions with topness are the same as the charmed baryons. The weak interactions follow from the standard assignment into doublets with the appropriate Kobayashi-Maskawa matrix.

The description of the charmed mesons needs the introduction of scalar fields for the bilinears $q \bar{c}, \bar{q} c, \bar{c} c$ with $q$ representing the three light quarks $(u, d, s)$. As for the light quark-antiquark pairs these scalars transform as singlets or octets with respect to the color symmetry $S U(3)_{C}$. From the transformation properties with respect to $S U(3)_{L} \times S U(3)_{R} \times S U(3)_{C}$ we can read the representations of the physical $S U(3)_{V}$-group

$$
\begin{aligned}
&(q \bar{c}):(3,1,8+1) \longrightarrow 3+3+\overline{6}+15 \\
&(1,3,8+1) \longrightarrow 3+3+\overline{6}+15 \\
&(c \bar{c}): \quad(1,1,8+1) \longrightarrow 8+1
\end{aligned}
$$

Only the singlet $(c \bar{c})$ can acquire a vacuum expectation value consistent with $S U(3)_{V}$-symmetry. We also note that a direct Yukawa coupling of 
the charmed quark to $\phi$ or $\chi$ is not allowed by the chiral $S U(3)_{L} \times S U(3)_{R}$ symmetry. The relation between current quark mass and baryon mass (or constituent quark mass) may therefore differ between charmed quarks and up, down, or strange quarks. In summary, the charmed particles do not constitute an obstacle for the picture of spontaneous breaking of color. The quantum numbers of the physical fields agree with those of observed particles. The same holds for beauty and top.

\section{Conclusion}

We conclude that the "spontaneous breaking" of color is compatible with observation. The simple effective action (2.6) gives a realistic approximate description of the masses of all low-lying mesons and baryons and of their interactions. Gluon-meson duality is associated to the well-known Higgs phenomenon with colored composite scalar fields, corresponding to quarkantiquark pairs.

The most important characteristics of our scenario can be summarized in the following points.

(i) The Higgs mechanism generates a mass for the gluons. In the limit of equal (current) masses for the three light quarks the masses of all gluons are equal. This leads to a simple picture of confinement: If one places color charges at different positions in the vacuum, the gauge fields cannot vanish. Due to the nonzero mass of the gauge fields, the energetically most favorable configurations are color-magnetic and color-electric flux tubes. This is in complete analogy with the Meissner effect in superconductors, with the additional ingredient that the Yang-Mills self-interactions link color-electric and color-magnetic fields. The string tension of the flux tubes provides for a simple mechanism for the confinement of color charges. More precisely, these statements hold only as long as string breaking due to quark-antiquark pairs is energetically suppressed. One may attempt a computation of the string tension appearing in the heavy quark potential by solving the field equations derived from the effective action (2.6) in presence of static color charges.

(ii) The physical fermion fields are massive baryons with integer electric charge. The low mass baryons form an octet with respect to the $S U(3)$ symmetry group of the "eightfold way" In a gauge-invariant language the baryons are quarks with a dressing of nonlinear fields. In a gauge-fixed version quarks and baryons can be described by the same field. This is quark-baryon duality. The main contribution to the mass of these baryons arises from chiral symmetry breaking through quark-antiquark condensates in the color singlet and octet channels. These considerations extend to the heavy quarks $c, b, t$, except that the mass is now dominated by the current quark mass. The lightest charmed baryons (and $t$-baryons) belong to a $S U(3)$-antitriplet with

electric charge $0,1,1$. Correspondingly, the $S U(3)$-antitriplet of the lightest 
b-baryons carries electric charge $-1,0,0$.

(iii) Our scenario shares the properties of chiral symmetry breaking with many other approaches to long-distance strong interactions. In particular, chiral perturbation theory is recovered in the low energy limit. This guarantees the observed mass pattern for pions, kaons and the $\eta$-meson and the structure of their (low momentum) interactions.

(iv) Spontaneous color symmetry breaking generates a nonlinear local $S U(3)_{P}$-reparametrization symmetry. The gauge bosons of this symmetry originate from the gluons. They form the octet of low masses physical vector mesons. (The singlet discussed in sect. 8 can be associated with the gauge boson of the abelian $U(1)_{P}$-symmetry and the ninth vector meson.) The symmetry relations following from $S U(3)_{P}$ symmetry appear in the electromagnetic and strong interactions of the vector mesons. They are compatible with observation and provide for a successful test of our scenario.

(v) The $\Delta I=1 / 2$ rule for the weak hadronic kaon decays arises naturally once weak interactions are incorporated into our picture. It is a consequence of the properties of the lowest dimension operators which are consistent with the symmetries.

Beyond the important general symmetry relations arising from the nonlinear local reparametrization symmetry the simple effective action (2.6) leads to particular predictions. They are related to the assumption that the effective action can be described in leading order by effective couplings with positive or zero mass dimension. This should hold once composite scalar fields are introduced for quark-antiquark bilinears and counted according to the canonical dimension for scalar fields. Expressed in other words we assume that the dominant nonperturbative effects in QCD result in the formation of scalar and pseudoscalar bound states and in large effective couplings. This assumption of scalar Vervollständigung can be tested by comparing the predictions of the effective action (2.6) with observation. For this purpose we fix the parameters $\chi_{0}, \sigma_{0}$ and $g$ by $M_{\rho}, f_{\pi}$ and $\Gamma\left(\rho \rightarrow e^{+} e^{-}\right)$. More precisely, we use here eqs. (5.16), (5.17) (7.14) with $\bar{M}_{\rho}=850 \mathrm{MeV}, f_{0}=128 \mathrm{MeV}$, $g_{\rho \gamma}=0.12 \mathrm{GeV}^{2}$. (With $g=6$ we may take the limit $x \rightarrow \infty$ for many expressions, keeping in mind that the precise value of $x$ is needed mainly for a determination of $\sigma_{0}$.) In addition, the Yukawa couplings $h, \tilde{h}$ are fixed by the baryon masses $M_{8}, M_{1}$ (cf. eq. (4.10)) whereas the strength of the chiral anomaly $\nu$ and $\nu^{\prime}$ determines $M_{\eta^{\prime}}^{2}$ by eq. (5.19). (The precise ratio $\nu^{\prime} / \nu$ is not relevant here.) We concentrate on the following predictions of scalar Vervollständigung which are not dictated purely by symmetry considerations:

(1) The pion nucleon couplings are found as $F=D=0.5$ to be compared with the observed values $F=0.459 \pm 0.008, D=0.798 \pm 0.008$.

(2) The decay width $\Gamma(\rho \rightarrow 2 \pi)=115 \mathrm{MeV}$ turns out to be somewhat lower than the observed value of $150 \mathrm{MeV}$. (Note that we use here directly eq. (8.2) and $\kappa_{f}$ equals one in leading order, such that $g_{\rho \pi \pi}=4.6$.)

(3) The direct coupling of the photon to pions is suppressed $g_{\gamma \pi \pi} / e=0.04$ 
(by virtue of eq. (7.17) with $M_{\rho} \rightarrow \bar{M}_{\rho}, f_{\pi} \rightarrow f_{0}$ ). This phenomenon of vector dominance describes well the observations.

(4) The effective next-to leading order couplings $L_{1}, L_{2}, L_{3}$ of chiral perturbation theory come out compatible with observation. In particular, scalar Vervollständigung predicts $L_{2}=1.7 \cdot 10^{-3}, L_{3}=-(3.9-5.2) \cdot 10^{-3}$.

(5) The inclusion of weak interactions leads to a suppression of the $\Delta I=$ $3 / 2$ hadronic kaon decays compared to the ones with $\Delta I=1 / 2$. A realistic picture of the three hadronic kaon two-body decays is obtained. This involves, however, two additional parameters.

For a first approximation we consider the effective action (2.6) as very satisfactory. Corrections arise from two sources: (a) The nonvanishing quark masses, in particular the strange quark mass, break the physical global $S U(3)$-symmetry. (b) Higher order invariants are certainly present in the full effective action. One important source relevant for the vector mesons is discussed in appendix A. Some of the corrections are known phenomenologically as, for example, the partial Higgs effect which reduces the average meson decay constant from $f_{0}$ to the mean value $f=\left(f_{\pi}+2 f_{K}\right) / 3=106 \mathrm{MeV}$. Together with the further lowering of $f_{\pi}$ as compared to $f$ by $S U(3)_{V}$ violation this leads to a realistic value of $\Gamma(\rho \rightarrow 2 \pi)$. The neglected higher-order invariants can be used to improve the agreement with observation. Typically, these corrections are below $30 \%$.

Once the singlet vector meson $S_{\mu}$ is added (cf. eq. (8.7)) the effective action (2.6) may be viewed as the lowest order of a twofold systematic expansion. First, higher ("non-renormalizable") invariants involving additional powers of $\phi$ or $\chi$ lead to corrections $\sim f^{2} / \mu_{s}^{2} \approx 0.1-0.3$ with $\mu_{s}$ a typical strong interaction scale. We have encountered this factor in hadronic weak decays $\left(\mu_{s} \hat{=} \mu_{W}\right)$, the partial Higgs effects (5.11), (A.41) $\left(\mu_{s} \hat{=} \mu_{\rho} / e_{\rho \pi \pi}\right)$ or the coupling between nucleons and the axial vector pion current $g_{A}-1 \sim y_{N}$ (9.2), (A.45) $\left(\mu_{s} \hat{=} \mu_{\rho} / \sqrt{c_{\rho \bar{q} q} e_{\rho \pi \pi}}\right)$. Second, the expansion in the number of derivatives involves some characteristic squared momentum $q^{2} / M_{s}^{2}$ as a small parameter. Typically $M_{s}$ may be of the size of the mass of particles not included in our description, i. e. $M_{s} \approx 1 \mathrm{GeV}$. A typical operator is $\left(Z_{\psi} / M_{s}\right) \bar{\psi}_{i}\left[\gamma_{\mu}, \gamma_{\nu}\right] G_{i j}^{\mu \nu} \psi_{j}$. This is responsible for the anomalous magnetic moment of the nucleons via the mixing between the photon and the gluon $\tilde{G}_{\mu}$ (see sects. 3,7). We observe a typical relation $M_{s} / \mu_{s}=c_{s}$ with $c_{s}$ around three a dimensionless coupling constant of the type $e_{\rho \pi \pi}$. Furthermore, the expansion in $S U(3)$ violating effects originating from the strange quark mass is governed [15] by $\left(f_{K}-f_{\pi}\right) / f \approx 0.2$. This last expansion may remain within the hypothesis of scalar Vervollständigung.

The parameters of the model being fixed additional predictions become possible. An example are the rare electromagnetic decays of mesons. Particularly interesting is the strangeness splitting of masses of the various $S U(3)_{V^{-}}$ muliplets. This involves, however, some additional parameters of the effective scalar potential (2.8). On a phenomenological basis the $S U(3)_{V}$-violation will 
apear in the form of different expectation values of $\phi$ and $\chi$ in the strange and nonstrange directions.

The present framework opens new perspectives for a calculation of the properties of hadronic matter at high temperature and density. Especially for high density the issue of baryons vs. quarks plays a crucial role due to the different Fermi surfaces [18]. Quark-baryon duality allows for a simple approach to this problem by using only one field.

Quark-baryon duality has profound implications. The nonrelativistic quark model where baryons are bound states of three quarks is now supplemented by the view of baryons as "dressed quarks". In a high energy scattering process "physical quarks" will come out, but they will come out with the quantum numbers and masses of baryons. In this sense, quarks are not confined particles, despite the fact that color charges remain exactly confined and cannot appear connected with free particles. When leaving the interaction region, the physical particles (asymptotic states) acquire always a dressing by (nonlinear) fields that makes them color neutral and integer charged. (In precise formulations of quantum field theory this also happens to electrons - the physical particles being neutral with respect to the electromagnetic gauge symmetry.) This view may have important consequences for our picture of the parton model, both for structure functions at small $Q^{2}$ and for fragmentation.

Let us finally address this perhaps most important open issue in our approach, i.e. its connection to the parton model at high $Q^{2}$ and to the nonrelativistic quark model which describes rather well the higher bound states. In short, one has to understand the correspondence between quarks dressed by a cloud of diquarks and bound states of three quarks. The effective action (2.6) is thought to be valid at low momenta. Going to higher momenta, the couplings become typically momentum-dependent. This also holds for the definition of the nonlinear fields, e.g. $Z_{\psi} \rightarrow Z_{\psi}\left(-D^{\mu} D_{\mu}\right)$ in eq. (4.1). Along the lines of the discussion in sect. 2 the effective action at high momentum (or, more precisely, large off-shell momentum) should be given by perturbative QCD, with $g$ the perturbative gauge coupling and $Z_{\psi}\left(-D^{2}\right) \approx 1$. The scalar part of the effective action should express appropriate 1PI contributions in the perturbative quark-gluon language. For high momentum scattering the dominant effect of spontaneous color symmetry breaking is then the mass term for the gluons which provides for an infrared cutoff. "Infrared safe" quantities which can be reliably described by perturbative QCD are not very sensitive to this mass.

In a momentum basis the nonlinear field decomposition (4.1) reads for $Z_{\psi}\left(-D^{2}\right)=1\left(\right.$ with $\left.\int_{p}=\int \frac{d^{4} p}{(2 \pi)^{4}}\right)$

$$
\psi_{L}(\tilde{p})=\int_{p} \int_{p^{\prime}} W_{L}\left(\tilde{p}-p-p^{\prime}\right) N_{L}(p) v\left(p^{\prime}\right)
$$


This may be inserted, for example, into the electromagnetic vertex

$$
\sim \tilde{e} \operatorname{Tr}\left\{\bar{\psi}(\tilde{p}+q) \gamma^{\mu} \tilde{Q} \psi(\tilde{p})\right\} \tilde{B}_{\mu}(q)
$$

The scattering of an off-shell photon with momentum $q$ and an off-shell quark with momentum $\tilde{p}$ to an off-shell quark with momentum $\tilde{p}+q$ describes the annihilation of an on-shell proton $N$ with momentum $p$ and simultaneous production of the fields contained in the "spectator jet" $Y(p-\tilde{p})$. Here the spectators consist of the particles described by the nonlinear fields $Y \sim W^{\dagger} \circ v^{\dagger}$, with $\circ$ denoting the appropriate algebra for the particular combination relevant for the proton. The "parton" $\psi(\tilde{p})$ carries a (longitudinal) fraction $x=-q^{2} /(2(p q)) \approx(\overrightarrow{\tilde{p}} \vec{p}) /\left(\vec{p}^{2}\right)$ of the spacelike momentum of the proton. Similarly, the produced quark $\psi(\tilde{p}+q)$ "fragments" into the hadrons $X$ contained in its nonlinear decomposition (13.1). In a process with high off-shell parton momenta final states involving many on-shell hadrons become kinematically accessible. On a hadronic level, this corresponds to inelastic multihadron production $N+\gamma^{*} \rightarrow X+Y$. If the parton structure functions can be suitably implemented in this setting ${ }^{27}$ the conservation of global quantum numbers should lead to the appropriate sum rules. A deviation of $Z_{\psi}$ from one modifies the composition of $X$ and $Y$ without effecting the general setting.

Instead of the momentum dependence of the effective couplings one may also investigate the related (but not identical!) dependence of the effective action $\Gamma_{k}$ on an appropriate infrared cutoff $k$ (see sect. 2). The use of nonperturbative flow equations [14] for $\Gamma_{k}$ may hopefully permit to connect perturbative QCD with the effective action $\Gamma_{0}(2.6)$.

These last ideas are only sketches for further developments. The phenomenological success of the simple low momentum effective action proposed in this paper should motivate a serious investigation in these directions. We hope that quark-baryon duality will permit further insights into the connection between the parton model and the QCD-vacuum.

\section{Acknowledgement}

This work was supported by the TMR-network FMRX-CT97-0122 and by the Deutsche Forschungsgemeinschaft We 1056/3-2.

\footnotetext{
${ }^{27}$ It is conceivable that the nonlinear constraints $v^{\dagger} v=1, W^{\dagger} W=1$ are not appropriate at high off-shell momenta. Fluctuations away from the nonlinear constraints may have to be included.
} 


\section{Appendix A: Four-quark interactions in the color singlet vector channel}

In this appendix we discuss the effects of four-quark interactions in the color singlet vector and axial vector channels. As for the scalar and pseudoscalar channels the effective action is described in a partially bosonized form. For this purpose we introduce fields for the composite quark-antiquark bilinears $\rho_{L}^{\mu}, \rho_{R}^{\mu}$ which correspond to $\bar{\psi}_{i} \gamma^{\mu}\left(1 \pm \gamma^{5}\right) \psi_{i}$.

Let us add to the effective action (2.6) a piece accounting for the fourquark interactions in the vector channel. We express it in terms of effective interactions ${ }^{28}$ for left-handed and right-handed vector fields $\rho_{L}^{\mu}, \rho_{R}^{\mu}$.

$$
\begin{aligned}
& \mathcal{L}_{\rho}=\mathcal{L}_{\rho \bar{q} q}+\mathcal{L}_{\rho^{2}}+\mathcal{L}_{\phi^{2} \rho}+\mathcal{L}_{\phi^{2} \rho^{2}}+\mathcal{L}_{\chi^{2} \rho}+\mathcal{L}_{\chi^{2} \rho^{2}}+\mathcal{L}_{\rho B} \\
& \mathcal{L}_{\rho \bar{q} q}=\sqrt{2} Z_{\psi} c_{\rho \bar{q} q} \operatorname{Tr}\left\{\bar{\psi}_{L} \gamma_{\mu} \tilde{\rho}_{L}^{\mu} \psi_{L}+\bar{\psi}_{R} \gamma_{\mu} \tilde{\rho}_{R}^{\mu} \psi_{R}\right\} \\
& +\frac{\sqrt{2}}{3} Z_{\psi} \tilde{c}_{\rho \bar{q} q}\left(\operatorname{Tr}\left\{\bar{\psi}_{L} \gamma_{\mu} \psi_{L}\right\} \operatorname{Tr} \rho_{L}^{\mu}+\operatorname{Tr}\left\{\bar{\psi}_{R} \gamma_{\mu} \psi_{R}\right\} \operatorname{Tr} \rho_{R}^{\mu}\right) \\
& \mathcal{L}_{\rho^{2}}=\left[\frac { 1 } { 2 } \operatorname { T r } \left\{\left(D_{\mu} \rho_{L \nu}-D_{\nu} \rho_{L \mu}\right)\left(D^{\mu} \rho_{L}^{\nu}-D^{\nu} \rho_{L}^{\mu}\right)\right.\right. \\
& \left.+\frac{1}{\alpha_{\rho}} \operatorname{Tr}\left\{\left(D_{\mu} \tilde{\rho}_{L}^{\mu}\right)\left(D_{\nu} \tilde{\rho}_{L}^{\nu}\right)\right\}+\frac{1}{3 \alpha_{\rho}^{\prime}}\left(\partial_{\mu} \operatorname{Tr} \rho_{L}^{\mu}\right)^{2}+\mu_{\rho}^{2} \operatorname{Tr}\left\{\tilde{\rho}_{L \mu} \tilde{\rho}_{L}^{\mu}\right\}+(L \leftrightarrow R)\right] \\
& +\frac{1}{6} \mu_{A}^{2} \operatorname{Tr}\left\{\rho_{L}^{\mu}-\rho_{R}^{\mu}\right\} \operatorname{Tr}\left\{\rho_{L \mu}-\rho_{R \mu}\right\}+\frac{1}{6} \mu_{V}^{2} \operatorname{Tr}\left\{\rho_{L \mu}^{\mu}+\rho_{R}^{\mu}\right\} \operatorname{Tr}\left\{\rho_{L \mu}+\rho_{R \mu}\right\} \\
& \mathcal{L}_{\phi^{2} \rho}=i \sqrt{2} c_{\rho \pi \pi} \operatorname{Tr}\left\{\left(D_{\mu} \phi^{\dagger} \phi-\phi^{\dagger} D_{\mu} \phi\right) \tilde{\rho}_{L}^{\mu}+\left(D_{\mu} \phi \phi^{\dagger}-\phi D_{\mu} \phi^{\dagger}\right) \tilde{\rho}_{R}^{\mu}\right\} \\
& +i \frac{\sqrt{2}}{3} \tilde{c}_{\rho \pi \pi} \operatorname{Tr}\left\{D_{\mu} \phi^{\dagger} \phi-\phi^{\dagger} D_{\mu} \phi\right\}\left(\operatorname{Tr} \rho_{L}^{\mu}-\operatorname{Tr} \rho_{R}^{\mu}\right) \\
& \mathcal{L} \phi^{2} \rho^{2}=2\left(c_{\rho \pi \pi}^{2}+f_{1}\right)\left(\operatorname{Tr}\left\{\phi^{\dagger} \phi \tilde{\rho}_{L}^{\mu} \tilde{\rho}_{L \mu}\right\}+\operatorname{Tr}\left\{\phi \phi^{\dagger} \tilde{\rho}_{R}^{\mu} \tilde{\rho}_{R \mu}\right\}\right) \\
& -4\left(c_{\rho \pi \pi}^{2}+f_{2}\right) \operatorname{Tr}\left\{\phi^{\dagger} \tilde{\rho}_{R}^{\mu} \phi \tilde{\rho}_{L \mu}\right\}-\frac{4}{3} \tilde{f}_{2}\left(\operatorname{Tr}\left\{\phi^{\dagger} \phi\right\}-3 \sigma_{0}^{2}\right) \operatorname{Tr} \rho_{R}^{\mu} \operatorname{Tr} \rho_{L \mu} \\
& +4 f_{3}\left(\operatorname{Tr}\left\{\phi^{\dagger} \phi\right\}-3 \sigma_{0}^{2}\right) \operatorname{Tr}\left\{\tilde{\rho}_{L}^{\mu} \tilde{\rho}_{L \mu}+\tilde{\rho}_{R}^{\mu} \tilde{\rho}_{R \mu}\right\} \\
& +\frac{4}{3} \tilde{f}_{3}\left(\operatorname{Tr}\left\{\phi^{\dagger} \phi\right\}-3 \sigma_{0}^{2}\right)\left(\operatorname{Tr} \rho_{L}^{\mu} \operatorname{Tr} \rho_{L \mu}+\operatorname{Tr} \rho_{R}^{\mu} \operatorname{Tr} \rho_{R \mu}\right) \\
& \mathcal{L}_{\chi^{2} \rho}=i \sqrt{2} e_{\rho \pi \pi}\left\{\left[\left(D_{\mu} \chi\right)_{i j a b}^{*} \chi_{i j a c}-\chi_{i j a b}^{*}\left(D_{\mu} \chi\right)_{i j a c}\right]\left(\tilde{\rho}_{L}^{\mu}\right)_{c b}\right. \\
& \left.+\left[\left(D_{\mu} \chi\right)_{i j a b} \chi_{i j c b}^{*}-\chi_{i j a b}\left(D_{\mu} \chi\right)_{i j c b}^{*}\right]\left(\tilde{\rho}_{R}^{\mu}\right)_{c a}\right\} \\
& +i \frac{\sqrt{2}}{3} \tilde{e}_{\rho \pi \pi}\left[\left(D_{\mu} \chi\right)_{i j a b}^{*} \chi_{i j a b}-\chi_{i j a b}^{*}\left(D_{\mu} \chi\right)_{i j a b}\right]\left(\operatorname{Tr} \rho_{L}^{\mu}-\operatorname{Tr} \rho_{R}^{\mu}\right)
\end{aligned}
$$

\footnotetext{
${ }^{28}$ We follow here closely appendix $\mathrm{B}$ of ref. 15]. The normalization of the matrix-valued fields $\rho_{L, R}^{\mu}$ used here differs by a a factor of two from 15.
} 


$$
\begin{aligned}
\mathcal{L}_{\chi^{2} \rho^{2}}= & e_{1}\left[\chi_{i j a b}^{*} \chi_{i j a c}\left(\tilde{\rho}_{L}^{\mu}\right)_{c d}\left(\tilde{\rho}_{L \mu}\right)_{d b}+\chi_{i j a b} \chi_{i j c b}^{*}\left(\tilde{\rho}_{R}^{\mu}\right)_{c d}\left(\tilde{\rho}_{R \mu}\right)_{d a}\right] \\
& +e_{2} \chi_{i j a b}^{*}\left(\tilde{\rho}_{R}^{\mu}\right)_{a c} \chi_{i j c d}\left(\tilde{\rho}_{L \mu}\right)_{d b} \\
& +\frac{1}{3} \tilde{e}_{2}\left(\chi_{i j a b}^{*} \chi_{i j a b}-\frac{4}{3} \chi_{0}^{2}\right) \operatorname{Tr} \rho_{R}^{\mu} \operatorname{Tr} \rho_{L \mu} \\
& +e_{3}\left(\chi_{i j a b}^{*} \chi_{i j a b}-\frac{4}{3} \chi_{0}^{2}\right) \operatorname{Tr}\left\{\tilde{\rho}_{L}^{\mu} \tilde{\rho}_{L \mu}+\tilde{\rho}_{R}^{\mu} \tilde{\rho}_{R \mu}\right\} \\
& +\frac{1}{3} \tilde{e}_{3}\left(\chi_{i j a b}^{*} \chi_{i j a b}-\frac{4}{3} \chi_{0}^{2}\right)\left(\operatorname{Tr} \rho_{L}^{\mu} \operatorname{Tr} \rho_{L \mu}+\operatorname{Tr} \rho_{R}^{\mu} \operatorname{Tr} \rho_{R \mu}\right) \\
\mathcal{L}_{\rho B}= & \frac{1}{6} e_{\rho \gamma \gamma} \operatorname{Tr}\left\{\partial_{\mu} \rho_{L \nu}-\partial_{\nu} \rho_{L \mu}+\partial_{\mu} \rho_{R \nu}-\partial_{\nu} \rho_{R \mu}\right\} \tilde{B}^{\mu \nu}
\end{aligned}
$$

Here we have defined

$$
\tilde{\rho}_{L, R}^{\mu}=\rho_{L, R}^{\mu}-\frac{1}{3} \operatorname{Tr} \rho_{L, R}^{\mu}=\frac{1}{2} \tilde{\rho}_{L, R}^{z \mu} \lambda_{z}
$$

and the electromagnetic covariant derivatives read

$$
D_{\mu} \rho_{L, R}^{\nu}=\partial_{\mu} \rho_{L, R}^{\nu}-i \tilde{e} B_{\mu}\left[\tilde{Q}, \rho_{L, R}^{\nu}\right]
$$

Up to omitted terms involving three or four vector fields $\rho_{L, R}^{\mu}$ this is the most general effective action with terms up to dimension four and consistent with chiral, color, electromagnetic and discrete symmetries. The fields $\rho_{L, R}^{\mu}$ are color-neutral and transform with respect to chiral $S U(3)_{L} \times S U(3)_{R}$ as octets $\tilde{\rho}_{L, R}^{\mu}$ and singlets $\operatorname{Tr} \rho_{L, R}^{\mu}$

$$
\delta \rho_{L \mu}=i\left[\Theta_{L}, \rho_{L}^{\mu}\right], \delta \rho_{R}^{\mu}=i\left[\Theta_{R}, \rho_{R}^{\mu}\right]
$$

whereas the discrete transformations read

$$
\begin{array}{ll}
P: & \rho_{L}^{\mu} \leftrightarrow \rho_{R}^{\mu} \\
C: & \rho_{L}^{\mu} \rightarrow-\left(\rho_{R}^{\mu}\right)^{T}, \quad \rho_{R}^{\mu} \rightarrow-\left(\rho_{L}^{\mu}\right)^{T}
\end{array}
$$

As discussed in sect. 2, the effective four-quark interactions in the vector channel (as well as other multiquark interactions) can be recovered from (A.1) by solving the field equations for $\rho_{L}^{\mu}$ and $\rho_{R}^{\mu}$ as functionals of $\psi, \phi$ and $\chi$. It is obvious that the reinsertion of this solution into the effective action produces an addition to the effective action (2.6) for $\psi, \phi, \chi$ and $A_{\mu}$ which now also contains terms of dimensions larger than four. In this sense the piece (A.1) can be interpreted as a suggestion for the most important higher order operators not yet contained in eq. (2.6). In particular, the induced effective four-quark interaction for low momenta reads

$$
\begin{aligned}
\Delta \mathcal{L}_{\rho}= & -Z_{\psi}^{2}\left[\tau_{V} \operatorname{Tr}\left\{\bar{\psi} \gamma^{\mu} \lambda_{z} \psi\right\} \operatorname{Tr}\left\{\bar{\psi} \gamma_{\mu} \lambda_{z} \psi\right\}\right. \\
& \left.-\tau_{A} \operatorname{Tr}\left\{\bar{\psi} \gamma^{\mu} \gamma^{5} \lambda_{z} \psi\right\} \operatorname{Tr}\left\{\bar{\psi} \gamma_{\mu} \gamma^{5} \lambda_{z} \psi\right\}\right] \\
& -\frac{Z_{\psi}^{2} \tilde{c}_{\rho \bar{q} q}^{2}}{12 \mu_{V}^{2}} \operatorname{Tr}\left\{\bar{\psi} \gamma^{\mu} \psi\right\} \operatorname{Tr}\left\{\bar{\psi} \gamma_{\mu} \psi\right\} \\
& -\frac{Z_{\psi}^{2} \tilde{c}_{\rho \bar{q} q}^{2}}{12 \mu_{A}^{2}} \operatorname{Tr}\left\{\bar{\psi} \gamma^{\mu} \gamma^{5} \psi\right\} \operatorname{Tr}\left\{\bar{\psi} \gamma_{\mu} \gamma^{5} \psi\right\}
\end{aligned}
$$


with

$$
\tau_{V}=\frac{c_{\rho \bar{q} q}^{2}}{8 M_{V}^{2}} \quad, \quad \tau_{A}=\frac{c_{\rho \bar{q} q}^{2}}{8 M_{A}^{2}}
$$

The first term contributes to the isospin triplet vector channel for the nucleonnucleon interactions (with $M_{V}$ and $M_{A}$ given below).

The effective interactions in (A.1) involve many new parameters. We concentrate here only on the most important modifications as compared to the effective action (2.6). First of all, the fields $\rho_{L}^{\mu}$ and $\rho_{R}^{\mu}$ contain vector $\left(r_{V}^{\mu}\right)$ and axial vector $\left(\rho_{A}^{\mu}\right)$ degrees of freedom

$$
r_{V}^{\mu}=\frac{1}{\sqrt{2}}\left(\rho_{R}^{\mu}+\rho_{L}^{\mu}\right), \quad \rho_{A}^{\mu}=\frac{1}{\sqrt{2}}\left(\rho_{R}^{\mu}-\rho_{L}^{\mu}\right)
$$

The squared mass for the axial vector octet obtains as

$$
M_{A}^{2}=\mu_{\rho}^{2}+2 \sigma_{0}^{2}\left(2 c_{\rho \pi \pi}^{2}+f_{1}+f_{2}\right)+\left(\frac{2}{9} e_{1}+\frac{1}{36} e_{2}\right) \chi_{0}^{2}
$$

whereas the axial vector singlet mass term is given by $\mu_{A}^{2}$. The vector field $r_{V}^{\mu}$ provides now for the missing singlet vector meson

$$
S_{\mu}=\sqrt{\frac{2}{3}} \operatorname{Tr} r_{V}^{\mu}
$$

with squared mass $\mu_{V}^{2}$. We note that the axial vectors cannot mix with the vectors because they have different parity. Also the singlets cannot mix with the octets in the limit of an unbroken physical $S U(3)$ symmetry. On the other hand, the octet in $r_{V}^{\mu}$ has the same transformation properties with respect to the physical global symmetries as the gluons. One therefore expects a mixing with the fields in $\tilde{V}^{\mu}$.

For an investigation of the interactions with the pseudoscalar mesons we introduce again nonlinear fields

$$
T_{L}^{\mu}=W_{L}^{\dagger} \rho_{L}^{\mu} W_{L}, \quad T_{R}^{\mu}=W_{R}^{\dagger} \rho_{R}^{\mu} W_{R}
$$

and we replace eq. (A.15) by

$$
r_{V}^{\mu}=\frac{1}{\sqrt{2}}\left(T_{R}^{\mu}+T_{L}^{\mu}\right), \quad \rho_{A}^{\mu}=\frac{1}{\sqrt{2}}\left(T_{R}^{\mu}-T_{L}^{\mu}\right)
$$

These fields transform homogeneously as octets and singlets under the local reparametrization symmetry

$$
\delta T_{L, R}^{\mu}=i\left[\Theta_{P}, T_{L, R}^{\mu}\right]
$$

and are neutral with respect to $S U(3))_{L} \times S U(3)_{R} \times S U(3)_{C}$. They also transform homogeneously with respect to the electromagnetic gauge symmetry

$$
\delta_{e m} r_{V}^{\mu}=i \beta\left[\tilde{Q}, r_{V}^{\mu}\right], \quad \delta_{e m} \rho_{A}^{\mu}=i \beta\left[\tilde{Q}, \rho_{A}^{\mu}\right]
$$


If we define

$$
\begin{aligned}
\hat{D}_{\mu} r_{V \nu}= & \partial_{\mu} r_{V \nu}-i e \tilde{B}_{\mu}\left[\tilde{Q}, r_{V \nu}\right]+i\left[\hat{v}_{\mu}, r_{V \nu}\right]+i\left[\hat{a}_{\mu}, \rho_{A \nu}\right] \\
\hat{D}_{\mu} \rho_{A \nu}= & \partial_{\mu} \rho_{A \nu}-i e \tilde{B}_{\mu}\left[\tilde{Q}, \rho_{A \nu}\right]+i\left[\hat{v}_{\mu}, \rho_{A \nu}\right]+i\left[\hat{a}_{\mu}, r_{V \nu}\right] \\
& \hat{a}_{\mu}=-\frac{i}{2}\left(W_{R}^{\dagger} D_{\mu} W_{R}-W_{L}^{\dagger} D_{\mu} W_{L}\right)
\end{aligned}
$$

the terms quadratic in $\rho$ read

$$
\begin{aligned}
\mathcal{L}_{\rho^{2}}= & \frac{1}{2} \operatorname{Tr}\left\{\left(\hat{D}_{\mu} r_{V \nu}-\hat{D}_{\nu} r_{V \mu}\right)\left(\hat{D}^{\mu} r_{V}^{\nu}-\hat{D}^{\nu} r_{V}^{\mu}\right)\right\} \\
& +\frac{1}{2} \operatorname{Tr}\left\{\left(\hat{D}_{\nu} \rho_{A \nu}-\hat{D}_{\nu} \rho_{A \mu}\right)\left(\hat{D}^{\mu} \rho_{A}^{\nu}-\hat{D}^{\nu} \rho_{A}^{\mu}\right)\right\} \\
& +\frac{1}{\alpha_{\rho}} \operatorname{Tr}\left\{\left(\hat{D}_{\mu} \tilde{r}_{V}^{\mu}\right)^{2}+\left(\hat{D}_{\mu} \tilde{\rho}_{A}^{\mu}\right)^{2}\right\} \\
& +\frac{1}{2 \alpha_{\rho}^{\prime}}\left[\left(\partial^{\mu} S_{A \mu}\right)^{2}+\left(\partial^{\mu} S_{\mu}\right)^{2}\right] \\
& +\mu_{\rho}^{2}\left(\operatorname{Tr}\left\{\tilde{r}_{V}^{\mu} \tilde{r}_{V \mu}\right\}+\operatorname{Tr}\left\{\tilde{\rho}_{A}^{\mu} \tilde{\rho}_{A \mu}\right\}\right) \\
& +\frac{1}{2} \mu_{A}^{2} S_{A}^{\mu} S_{A \mu}+\frac{1}{2} \mu_{V}^{2} S^{\mu} S_{\mu}
\end{aligned}
$$

with

$$
\tilde{r}_{V}^{\mu}=r_{V}^{\mu}-\frac{1}{3} \operatorname{Tr} r_{V}^{\mu}, \quad \tilde{\rho}_{A}^{\mu}=\rho_{A}^{\mu}-\frac{1}{3} \operatorname{Tr} \rho_{A}^{\mu}, \quad S_{A}^{\mu}=\sqrt{\frac{2}{3}} \operatorname{Tr} \rho_{A}^{\mu}
$$

The existence of an additional homogeneous vector field $r_{V}^{\mu}$ allows us to construct new invariants and leads to mixing. Indeed, the invariant for the vector currents in (7.1) is now enlarged by a piece

$$
\begin{aligned}
\mathcal{L}_{V V}= & \chi_{0}^{2} \operatorname{Tr}\left\{\left(\hat{v}_{\mu}+g \tilde{V}_{\mu}-e \tilde{B}_{\mu} \tilde{Q}\right)\left(\hat{v}^{\mu}+g \tilde{V}^{\mu}-e \tilde{B}^{\mu} \tilde{Q}\right)\right\} \\
& +M_{V}^{2} \operatorname{Tr}\left\{\tilde{r}_{V \mu} \tilde{r}_{V}^{\mu}\right\}+2 \nu_{\rho} \operatorname{Tr}\left\{\left(\hat{v}_{\mu}+g \tilde{V}_{\mu}-e \tilde{B}_{\mu} \tilde{Q}\right) \tilde{r}_{V}^{\mu}\right\}
\end{aligned}
$$

with

$$
M_{V}^{2}=\mu_{\rho}^{2}+2 \sigma_{0}^{2}\left(f_{1}-f_{2}\right)+\left(\frac{2}{9} e_{1}-\frac{1}{36} e_{2}\right) \chi_{0}^{2}
$$

The mixing term $\sim \nu_{\rho}$ arises from the covariant derivative in the cubic $\chi-$ $\chi-\rho \operatorname{term}\left(\hat{\hat{a}}_{\mu}=\hat{a}_{\mu}-\frac{1}{3} \operatorname{Tr} \hat{a}_{\mu}\right)$

$$
\begin{gathered}
\mathcal{L}_{\chi^{2} \rho}=-2 e_{\rho \pi \pi} \chi_{0}^{2}\left[\operatorname{Tr}\left\{\left(\hat{v}_{\mu}+g \tilde{V}_{\mu}-e \tilde{B}_{\mu} \tilde{Q}\right) \tilde{r}_{V}^{\mu}\right\}+\frac{7}{9} \operatorname{Tr}\left\{\tilde{\hat{a}}_{\mu} \tilde{\rho}_{A}^{\mu}\right\}\right] \\
\nu_{\rho}=-e_{\rho \pi \pi} \chi_{0}^{2}
\end{gathered}
$$

The low mass vector meson octet $\tilde{\rho}_{V}^{\mu}$ and a heavy state $\tilde{H}_{V}^{\mu}$

$$
\begin{aligned}
\tilde{\rho}_{V}^{\mu} & =\cos \tau \tilde{V}^{\mu}+\sin \tau \tilde{r}_{V}^{\mu}, \\
\tilde{H}_{V}^{\mu} & =\cos \tau \tilde{r}_{V}^{\mu}-\sin \tau \tilde{V}_{\mu}
\end{aligned}
$$


are the mass eigenstates with mass eigenvalues

$$
\begin{aligned}
M_{\rho}^{2} & =\frac{1}{2}\left(g^{2} \chi_{0}^{2}+M_{V}^{2}-\sqrt{\left(M_{V}^{2}-g^{2} \chi_{0}^{2}\right)^{2}+4 g^{2} \nu_{V \rho}^{2}}\right. \\
M_{H}^{2} & =\frac{1}{2}\left(g^{2} \chi_{0}^{2}+M_{V}^{2}+\sqrt{\left(M_{V}^{2}-g^{2} \chi_{0}^{2}\right)^{2}+4 g^{2} \nu_{V \rho}^{2}}\right.
\end{aligned}
$$

and mixing angle

$$
\operatorname{tg}(2 \tau)=-\frac{2 g \nu_{\rho}}{M_{V}^{2}-g^{2} \chi_{0}^{2}}
$$

For the interactions it is most convenient to eliminate $\tilde{r}_{V}^{\mu}$ by its field equation. In the local approximation, where the kinetic term for $\tilde{r}_{V}^{\mu}$ is small compared to the mass term $\left(q^{2} \ll M_{V}^{2}\right)$, one finds

$$
\tilde{r}_{V}^{\mu}=-\frac{\nu_{\rho}}{M_{V}^{2}}\left(\hat{v}^{\mu}+g \tilde{V}^{\mu}-e \tilde{B}^{\mu} \tilde{Q}\right)
$$

and therefore

$$
\mathcal{L}_{V V}=\left(\chi_{0}^{2}-\frac{\nu_{\rho}^{2}}{M_{V}^{2}}\right) \operatorname{Tr}\left\{\left(\hat{v}_{\mu}+g \tilde{V}_{\mu}-e \tilde{B}_{\mu} \tilde{Q}\right)\left(\hat{v}^{\mu}+g \tilde{V}^{\mu}-e \tilde{B}^{\mu} \tilde{Q}\right)\right\}
$$

This is the same structure as (7.1), leading for the $\rho-\pi$-interaction (7.5) to

$$
a=\frac{1}{f_{\pi}^{2}}\left(\chi_{0}^{2}-\frac{\nu_{\rho}^{2}}{M_{V^{2}}}\right)
$$

hereby lowering the value of $a$ as compared to $(7.6)$. The kinetic term for $\tilde{V}^{\mu}$ also obtains a contribution from the insertion of (A.33) such that the $\rho$-mesons with a standard normalization correspond to

$$
\tilde{\rho}_{V}^{\mu}=\left(1+\frac{g^{2} \nu_{\rho}^{2}}{M_{V}^{4}}\right)^{-1 / 2} \tilde{V}^{\mu}
$$

After a replacement of $\tilde{V}$ by $\tilde{\rho}_{V}$ this modifies $(7.6)$ according to

$$
g_{\rho}=g\left(1+\frac{g^{2} \nu_{\rho}^{2}}{M_{V}^{4}}\right)^{1 / 2}
$$

As announced before the structure of the interactions between vector mesons, pseudoscalar mesons and photons is dictated by the local $U(3)_{P} \times U(1)_{e m}$ symmetries. The low momentum limit is not modified by the inclusion of additional interactions or degrees of freedom. In the following we work in the approximation

$$
\nu_{\rho}^{2} \ll \chi_{0}^{2} M_{V}^{2}, \quad e_{\rho \pi \pi}^{2} \ll \frac{M_{V}^{2}}{\chi_{0}^{2}}
$$

such that the modifications of $a$ and $g_{\rho}$ can be neglected. 
Let us next turn to the contribution to the pseudoscalar kinetic term (5.11) from the interaction $\sim \operatorname{Tr}\left\{\tilde{\rho}_{A}^{\mu} \tilde{\hat{a}}_{\mu}\right\}$. In addition to (A.28) such a term is also induced by

$$
\mathcal{L}_{\phi^{2} \rho}=-8 c_{\rho \pi \pi} \sigma_{0}^{2} \operatorname{Tr}\left\{\tilde{\rho}_{A}^{\mu} \tilde{\hat{a}}_{\mu}\right\}
$$

With the electromagnetically covariant axial vector current

$$
\begin{aligned}
\hat{a}_{\mu} & =a_{\mu}+\frac{e}{2} \tilde{B}_{\mu}\left(W_{L}^{\dagger} Q W_{L}-W_{R}^{\dagger} Q W_{R}\right) \\
& =-\frac{i}{2} W_{R}^{\dagger} D_{\mu} U W_{L}=\frac{i}{2} W_{L}^{\dagger} D_{\mu} U^{\dagger} W_{R} \\
\tilde{\hat{a}}_{\mu} & =\hat{a}_{\mu}-\frac{1}{3} \operatorname{Tr} \hat{a}_{\mu}, \quad \operatorname{Tr} \hat{a}_{\mu}=\operatorname{Tr} a_{\mu}=-\frac{1}{2} \partial_{\mu} \theta
\end{aligned}
$$

the term linear in $\tilde{\rho}_{A}^{\mu}$ is also linear in the pseudoscalar fields. It corresponds to a mixing between the pseudoscalar in $U$ and the pseudoscalar $\partial_{\mu} \rho_{A}^{\mu}$ (both are $0^{-+}$states). This so-called "partial Higgs effect" has been extensively discussed in [15]. After elimination of the field $\rho_{A}^{\mu}$ by virtue of the field equation it leads to an additional negative contribution to the pion decay constant $f$. Indeed, for

$$
\begin{aligned}
\mathcal{L}_{\rho A} & =M_{A}^{2} \operatorname{Tr}\left\{\tilde{\rho}_{A}^{\mu} \tilde{\rho}_{A \mu}\right\}+2 \alpha \operatorname{Tr}\left\{\tilde{\rho}_{A}^{\mu} \hat{a}_{\mu}\right\}-c_{\rho \bar{q} q} \operatorname{Tr}\left\{\bar{N} \gamma_{\mu} \gamma^{5} \tilde{\rho}_{A}^{\mu} N\right\}+\ldots \\
\alpha & =-4 \sigma_{0}^{2}\left(c_{\rho \pi \pi}+x e_{\rho \pi \pi}\right)
\end{aligned}
$$

the insertion of the solution of the field equation

$$
\tilde{\rho}_{A}^{\mu}=-\frac{\alpha}{M_{A}^{2}} \tilde{\hat{a}}^{\mu}+\frac{c_{\rho \bar{q} q}}{4 M_{A}^{2}} \operatorname{Tr}\left\{\bar{N} \gamma^{\mu} \gamma^{5} \lambda_{z} N\right\} \lambda_{z}+\ldots
$$

yields the bosonic contribution

$$
\mathcal{L}_{\rho A}^{(1)}=-\frac{\alpha^{2}}{M_{A}^{2}} \operatorname{Tr}\left\{\tilde{\hat{a}}^{\mu} \tilde{\hat{a}}_{\mu}\right\}+\ldots=-\frac{\alpha^{2}}{4 M_{A}^{2}} \operatorname{Tr}\left\{D^{\mu} \tilde{U}^{\dagger} D_{\mu} \tilde{U}\right\}+\ldots
$$

This constitutes the contribution $\Delta_{f}^{2}$ mentioned in sect. 5

$$
\frac{\Delta_{f}^{2}}{f^{2}}=\frac{\alpha^{2}}{M_{A}^{2} f^{2}}=\frac{\left(c_{\rho \pi \pi}+x e_{\rho \pi \pi}\right)^{2}}{(1+x)^{2}} \frac{f^{2}}{\kappa_{f}^{4} M_{A}^{2}} \approx 0.45
$$

Similarly, the contribution quadratic in the baryon fields reads

$$
\begin{aligned}
\mathcal{L}_{\rho A}^{(2)} & =y_{N} \operatorname{Tr}\left\{\bar{N} \gamma_{\mu} \gamma^{5} \tilde{\hat{a}}^{\mu} N\right\} \\
y_{N} & =\frac{\alpha c_{\rho \bar{q} q}}{M_{A}^{2}} \approx 0.45 \frac{c_{\rho \bar{q} q} f^{2}}{\alpha} \approx-0.31 \frac{c_{\rho \bar{q} q}(1+x)}{c_{\rho \pi \pi}+x e_{\rho \pi \pi}}
\end{aligned}
$$

There are similar contributions from the elimination of the singlet axial vector $\operatorname{Tr} a^{\mu}$. 
We finally note that the insertion? of eq. (A.42) into (A.24) introduces a term (after partial integration)

$$
\begin{aligned}
\mathcal{L}_{\rho 2} & =-2 i \frac{\alpha}{M_{A}^{2}} \operatorname{Tr}\left\{\left(D^{\mu} r_{V}^{\nu}-D^{\nu} r_{V}^{\mu}\right)\left[\hat{a}_{\mu}, \hat{a}_{\nu}\right]\right\}+\ldots \\
& =2 i \frac{\alpha}{M_{A}^{2} f^{2}} \operatorname{Tr}\left\{\partial^{2} r_{V T}^{\nu}\left[\Pi, \partial_{\nu} \Pi\right]\right\}+\ldots
\end{aligned}
$$

with $r_{V T}^{\nu}$ the transversal part of $r_{V}^{\nu}\left(\partial^{2}=\partial_{\mu} \partial^{\mu}\right)$

$$
r_{V T}^{\nu}=r_{V}^{\nu}-\frac{\partial^{\nu} \partial^{\rho}}{\partial^{2}} r_{V}^{\rho}
$$

Using (A.34), this replaces for the $\rho \rightarrow 2 \pi$ decay rate

$$
\begin{aligned}
g_{\rho \pi \pi} & \rightarrow g_{\rho \pi \pi}+\frac{\nu_{\rho} \alpha g}{M_{A}^{2} f^{2}} \\
& =g_{\rho \pi \pi}+\frac{9}{7} g \frac{x}{(1+x)^{2}} \frac{e_{\rho \pi \pi}\left(c_{\rho \pi \pi}+x e_{\rho \pi \pi}\right) f^{2}}{M_{A}^{2} \kappa_{f}^{4}} \\
& =g_{\rho \pi \pi}+0.45 \frac{\nu_{\rho}}{\alpha} g=g_{\rho \pi \pi}+0.58\left(1+\frac{c_{\rho \pi \pi}}{x e_{\rho \pi \pi}}\right)^{-1} g
\end{aligned}
$$

where we have employed the on-mass-shell condition $\left(\partial^{2} \rightarrow M_{V}^{2}\right)$. As a result, the phenomenologically required value of $g_{\rho \pi \pi}$ in eq. (7.8) could be smaller than 6 , and in consequence, the value of $a$ required by eq. (7.10) could be somewhat lower than 2 .

We observe that after eliminating $r_{V}^{\mu}$ by $($ A.33) the term (A.46) leads to an invariant

$$
\begin{aligned}
I_{3} & =\operatorname{Tr}\left\{\left(D^{\mu} v_{I}^{\nu}-D^{\nu} v_{I}^{\mu}\right)\left[\hat{a}_{\mu}, \hat{a}_{\nu}\right]\right\} \\
v_{I}^{\mu} & =\hat{v}^{\mu}+g \tilde{V}^{\mu}-e \tilde{B}^{\mu} \tilde{Q} \\
D_{\mu} v_{I \nu} & =\partial_{\mu} v_{I \nu}-i e \tilde{B}_{\mu}\left[\tilde{Q}, v_{I \nu}\right]+i\left[\hat{v}_{\mu}, v_{I \nu}\right]
\end{aligned}
$$

Since $v_{I}^{\mu}$ and $\hat{a}^{\mu}$ transform homogeneously with respect to $U(3)_{P} \times U(1)_{e m}$ and (A.50) involves a fully covariant derivative, it is obvious that $I_{3}$ adds a new invariant structure to the terms contained in eq. (7.1). This invariant influences the cubic and higher interactions but does not contribute to the two-point functions. Concerning eq. (7.8), it does not affect $M_{\rho}^{2}, g_{\gamma \rho}$ and $m_{B}^{2}$, whereas quantities like $g_{\rho \pi \pi}$ or $g_{\gamma \pi \pi}$ receive corrections. One concludes that the KSFR relation (7.9) is not a pure symmetry relation. Its validity also requires that the effective action for $\rho$-mesons is dominated by the invariant $\mathcal{L}_{V V}$ (7.5). The observed success of the KSFR relation implies that corrections from $I_{3}$ must be small. This is the case for $e_{\rho \pi \pi} \ll c_{\rho \pi \pi} / x$, implying that the mixing between $\tilde{r}_{V}^{\mu}$ and $\tilde{V}^{\mu}$ can indeed be neglected (cf. eq. (A.29)). For processes not involving axial vectors we therefore retain from (A.1) only $\mathcal{L}_{\rho^{2}}$ (for the singlet vector meson), $\mathcal{L}_{\phi^{2} \rho}$ (for the partial Higgs effect) and $\mathcal{L}_{\rho \bar{q} q}$ (for the effective nucleon interactions in the vector channel (A.13). This leads to the additional terms (8.7) displayed in sect. 8.

\footnotetext{
${ }^{29}$ The contributions from inserting corrections $\sim r_{V} \hat{a}$ to (A.42) into A.41) vanish.
} 


\section{Appendix B}

In this appendix we discuss invariants with $S U(3)_{C} \times S U(2)_{L} \times U(1)_{Y}$ local and $S U(3)_{R}$ global symmetry beyond the ones mentioned in sect. 10. We first turn to terms without derivatives. One may ask if local terms not involving derivatives could generate a term involving only one strange meson as, for example, the $C P$-even state $K_{L}^{0}=\frac{1}{\sqrt{2}}\left(K^{0}+\bar{K}^{0}\right)$. Such terms would contribute to the weak kaon decays. The answer is negative, as can be seen by inserting into the infinitesimal transformation of $\Pi$ up to linear order

$$
\delta \Pi=\frac{f}{2}\left(\Theta_{R}-\Theta_{L}\right)+i\left(\Theta_{R} \Pi-\Pi \Theta_{L}\right)+0\left(\Pi^{2}\right)
$$

the appropriate global $S U(2)_{R} \times U(1)_{R}$ or local $S U(2)_{L} \times U(1)_{Y}$ transformations. In fact, the remaining symmetry is still powerful enough to forbid for $s_{\theta}=0$ any nonderivative terms involving the pseudoscalars $\pi^{ \pm}, \pi^{0}, \eta, K^{0}, \bar{K}^{0}$. Any nonderivative coupling must therefore involve an even number of charged kaons of the form $\left(K^{+} K^{-}\right)^{n}$. For $s_{\theta} \neq 0$ this is replaced by $\left(c_{\theta} K^{+}-s_{\theta} \pi^{+}\right)$. $\left(c_{\theta} K^{-}-s_{\theta} \pi^{-}\right)^{n}$. On the other hand, strangeness-violating processes always have to involve charged $W^{ \pm}$-bosons. In lowest order $\sim M_{W}^{-2}$ we can neglect the exchange of $Z^{0}$. The strangeness-violating short-distance four-quark interactions have then a global $S U(3)_{R}$ flavor symmetry. This forbids also the terms mentioned above. One infers that there are no strangeness-violating non-derivative interactions in order $M_{W}^{-2}$. We conclude that we can omit all additional non-derivative interactions for a discussion of the weak decays of pseudoscalars.

We next turn to terms involving two derivatives and we restrict the discussion for simplicity to $\phi$. It is straightforward to see that terms involving only one trace and only one matrix $\lambda_{W}$ lead to the same structure as (10.4) since $\phi^{\dagger} \phi=\sigma_{0}^{2}$ as far as the pseudoscalars are concerned. We therefore omit such terms. The invariant involving four powers of $\phi$ and one factor of $\lambda_{W}$ is proportional $\operatorname{Tr}\left\{\phi^{\dagger} D^{\mu} \phi \lambda_{W}\right\} \operatorname{Tr}\left\{\phi^{\dagger} D_{\mu} \phi\right\}$ and does not contribute to the hadronic kaon decays into two pions. This is obvious by expanding for $\vartheta=0$

$$
\phi^{\dagger} D_{\mu} \phi=\sigma_{0}^{2} \tilde{U}^{\dagger} \partial_{\mu} \tilde{U}=\frac{2 \sigma_{0}^{2}}{f}\left(i \partial_{\mu} \Pi+\frac{1}{f}\left[\partial_{\mu} \Pi, \Pi\right]\right)+\ldots
$$

A relevant invariant reads

$$
\mathcal{L}_{W, \phi}^{(4)}=-\frac{z_{W}^{\phi} g_{W}^{2}}{8 M_{W}^{2}} \operatorname{Tr}\left\{\left(\phi^{\dagger} D_{\mu} \phi-D_{\mu} \phi^{\dagger} \phi\right) \vec{\tau}_{W}\right\} \operatorname{Tr}\left\{\left(\phi^{\dagger} D^{\mu} \phi-D^{\mu} \phi^{\dagger} \phi\right) \vec{\tau}_{W}\right\}
$$

with

$$
\tau_{W 1}=c_{\theta} \tau_{1}+s_{\theta} \lambda_{4}, \quad \tau_{W 2}=c_{\theta} \tau_{2}+s_{\theta} \lambda_{5}, \quad \tau_{W 3}=\tau_{3}
$$

Here the fact that $\tau_{W 3}$ is not rotated is related to the absence of flavorchanging neutral currents. (The rotation of the type (10.2) has to be realized 
by a four by four matrix including the charm quark.) Up to a factor $-z_{W}^{\phi}$ the invariant $(\mathbb{B} .3)$ has precisely the same structure as the one generated from one-particle reducible $W$-exchange by inserting the field equation for $W$ as a functional of $\phi$. This is no surprise since the underlying structure on the quark level corresponds to box-type diagrams where the exchange of a $W$-boson is supplemented by gluon exchange. A similar argument holds for operators involving $\chi$. The effects of the weak mixing between scalars and pseudoscalars may also be represented by effective higher-order operators of the type $($ B.3 $)$ which obtain after elimination of the scalar fields via their field equation. We include all these effects $\$ 0$ by multiplying the $W$-boson exchange contribution (10.36) by a factor $\left(1-z_{W}\right)$. The relative minus sign $\left(z_{W}>0\right)$ reflects the negative sign of the corresponding anomalous dimension in perturbation theory.

Strangeness-violating local interactions can also appear on the level of the Yukawa couplings between quarks and mesons

$$
\mathcal{L}_{\beta}=Z_{\psi} \bar{\psi}_{R i}\left(\frac{g_{W}^{2} \beta_{W}^{2} h}{4 M_{W}^{2}} \phi \delta_{i j}+\frac{g_{W}^{2} \tilde{\beta}_{W}^{2} \tilde{h}}{4 M_{W}^{2}} \chi_{i j}\right) \lambda_{W} \psi_{L j}+h . c .
$$

The interaction $(\mathbb{B} .5)$ is the only dimensionless invariant contributing to strangeness-violating baryon interactions. According to the hypothesis of scalar Vervollständigung it should dominate the hyperon decays. It has to be supplemented by strangeness-violating mixings of the pseudoscalars discussed in appendix C.

We recall that we work in a basis where both the kinetic and the mass term for the quarks conserve strangeness. This imposes constraints on the effective strangeness-violating couplings. As discussed in sect. 2, we can recover the multi-quark interactions by inserting the solutions of the field equations for $\phi$ and $\chi$ as functionals of $\bar{\psi} \psi$. The terms (10.3), (10.4), (B.5) result in a shift of the solution $\phi[\psi]=\phi_{0}[\psi]+\delta \phi[\psi]$. For large enough $k$ this reads approximately

$$
\delta \phi_{a b}=\frac{g_{W}^{2}}{4 M_{W}^{2}}\left(m_{\phi}^{2}-\partial^{2}\right)^{-1}\left[Z_{\psi} \beta_{W}^{2} h \bar{\psi}_{L, i c}\left(\lambda_{W}\right)_{c b} \psi_{R, a i}-\left(\tilde{\mu}_{W}^{4}-\mu_{W}^{2} \partial^{2}\right)\left(\phi_{0} \lambda_{W}\right)_{a b}\right]
$$

with

$$
\left(\phi_{0}\right)_{a b}=Z_{\psi} h\left(m_{\phi}^{2}-\partial^{2}\right)^{-1} \bar{\psi}_{L i b} \psi_{R a i}+\frac{1}{2} Z_{\phi}^{-1 / 2} j_{a b}
$$

Insertion in the effective action (including the source term (2.9)) leads to a strangeness-conserving quark mass term only for $\beta_{W}^{2}=\tilde{\mu}_{W}^{4} m_{\phi}^{-2}$. A similar, but more complicated relation exists between the strangeness violation in the effective potential for $\phi, \chi$ and the strangeness violation in the Yukawa-type couplings.

\footnotetext{
${ }^{30}$ There are also contributions to the $\Delta I=1 / 2$ amplitude which may be absorbed by a small change in $\mu_{W}$.
} 
The insertion of (B.6) in the effective action leads to four-quark interactions involving two left-handed and two right-handed quarks. Assume that $\tilde{\mu}_{W}(k=0)$ can be related to $\tilde{\mu}_{W}(k)$ at a typical transition scale around 1 $\mathrm{GeV}$ by following the renormalization group flow of the effective action. It can then be matched to the perturbatively (RG-improved) computed value of the corresponding four-quark interaction. Since a tree exchange of $W$ bosons always involves at least four left-handed fermions, it is obvious that the four-quark interaction of interest can only be generated by a $W$-boson loop, as appropriate for a one-particle irreducible contribution. We will not pursue this road for a computation of the effective couplings $\mu_{W}, \tilde{\mu}_{W}$ etc. in the present work.

\section{Appendix C: $K_{L}^{0}-K_{S}^{0}$-mass difference}

For the discussion of the mass difference between $K_{S}^{0}$ and $K_{L}^{0}$ one needs the one-particle irreducible $\Delta S=2$ contributions in order $M_{W}^{-4}$

$$
\begin{aligned}
\mathcal{L}_{\mu}^{(\Delta S=2)}= & \frac{g_{W}^{4} \mu_{W, 2}^{2}}{16 M_{W}^{4}}\left(\eta_{1} \operatorname{Tr}\left\{\phi^{\dagger} D^{\mu} \phi \lambda_{W} \phi^{\dagger} D_{\mu} \phi \lambda_{W}\right\}\right. \\
& \left.+\eta_{2} \operatorname{Tr}\left\{\phi^{\dagger} D^{\mu} \phi \lambda_{W}\right\} \operatorname{Tr}\left\{\phi^{\dagger} D_{\mu} \phi \lambda_{W}\right\}\right)+\ldots
\end{aligned}
$$

Here the dots stand for terms involving $\chi$. This effective interaction corresponds to an eight-quark interaction. It is related to a vertex with four left-handed quarks (e.g. box diagrams with exchange of two $W$-boson lines) by supplementing the four right-handed quarks needed to form scalars. For the kinetic term of the pseudoscalar mesons it reduces to

$$
\begin{aligned}
\mathcal{L}_{\mu}^{(\Delta S=2)}= & \frac{3}{16} A_{W}^{2} \mu_{W, 2}^{2}\left[\eta_{1} \operatorname{Tr}\left\{\partial^{\mu} U \lambda_{6} \partial_{\mu} U \lambda_{6}\right\}\right. \\
& \left.+\eta_{2} \operatorname{Tr}\left\{\partial^{\mu} U \lambda_{6}\right\} \operatorname{Tr}\left\{\partial_{\mu} U \lambda_{6}\right\}\right]+\ldots
\end{aligned}
$$

This induces a difference in the wave function renormalization for $K_{L}^{0}$ and $K_{S}^{0}$

$$
\begin{aligned}
\mathcal{L}_{k i n} & =\frac{Z_{L}}{2} \partial^{\mu} K_{L}^{0} \partial_{\mu} K_{L}^{0}+\frac{Z_{S}}{2} \partial^{\mu} K_{S}^{0} \partial_{\mu} K_{S}^{0}+\ldots \\
Z_{L}-Z_{S} & =-\frac{3}{2} \frac{A_{W}^{2} \mu_{W, 2}^{2}}{f^{2}} \bar{\eta} \\
\bar{\eta} & =\eta_{1}+\eta_{2}+\ldots
\end{aligned}
$$

where the dots in $\bar{\eta}$ stand again for contributions from invariants involving the octet $\chi$. We normalize $\mu_{W 2}^{2}$ such that $\bar{\eta}=1$. Using a normalization with $Z_{S}=1, M_{K_{S}}=M_{K}$, one has $M_{K_{L}}=M_{K} Z_{L}^{-1 / 2}$ or

$$
\left(M_{K_{L}}-M_{K_{S}}\right)_{d}=\frac{3}{4} \frac{A_{W}^{2} \mu_{W 2}^{2} M_{K}}{f^{2}}=0.59 \cdot 10^{-12} \mathrm{MeV}\left(\frac{\mu_{W}^{2}}{f^{2}}\right)
$$


to be compared with the observed value

$$
M_{K_{L}}-M_{K_{S}}=3.522 \cdot 10^{-12} \mathrm{MeV}
$$

The index $d$ in eq. (C.4) indicates that this is only the direct contribution from the 1PI effective vertices. A further contribution arises from mixing effects of the pseudoscalar mesons in first order in $\Delta S$. In fact, due to weak interaction effects the fields in eq. (5.27) have small off-diagonal corrections in their propagators. Expanding $\mathcal{L}_{\mu U}(10.5)$ in second order in $\Pi, \theta$, one finds

$$
\begin{aligned}
\mathcal{L}_{\mu U}^{(2)}= & 2 A_{W} \hat{g}_{8}\left[\operatorname{Tr}\left\{\partial^{\mu} \Pi \partial_{\mu} \Pi \lambda_{6}\right\}-\frac{f}{3} \frac{1+\delta_{W} x}{1+5 \delta_{W} x / 14} \partial^{\mu} \theta \operatorname{Tr}\left\{\partial_{\mu} \Pi \lambda_{6}\right\}\right] \\
= & A_{W} \hat{g}_{8}\left[\partial^{\mu} \tilde{K}^{+} \partial_{\mu} \tilde{\pi}^{-}+\partial^{\mu} \tilde{\pi}^{+} \partial_{\mu} \tilde{K}^{-}-\partial^{\mu} \tilde{\pi}^{0} \partial_{\mu} \tilde{K}_{L}^{0}-\frac{1}{\sqrt{3}} \partial^{\mu} \tilde{\eta} \partial_{\mu} \tilde{K}_{L}^{0}\right] \\
& -\tilde{g}_{8} \partial^{\mu} \tilde{\eta}^{\prime} \partial_{\mu} \tilde{K}_{L}^{0} \\
\hat{g}_{8}= & \frac{14+5 \delta_{W} x}{14-4 \delta_{W} x} g_{8} \quad, \quad \tilde{g}_{8}=\frac{2 f}{3 H_{\eta^{\prime}}} \frac{1+\delta_{W} x}{1-2 \delta_{W} x / 7} g_{8}
\end{aligned}
$$

Here we use a tilde for the fields appearing in eq. (5.27) which correspond to the basis where the quark mass term $(2.9)$ is diagonal. The physical meson fields $\pi^{0}, K_{L, S}^{0}, \eta, \eta^{\prime}$ and $\pi^{ \pm}, K^{ \pm}$obtain after diagonalization of the inverse propagator. The total quadratic term for the neutral mesons reads

$$
\begin{aligned}
\mathcal{L}_{(2)}^{0}= & \frac{1}{2}\left(\partial^{\mu} \tilde{\pi}^{0} \partial_{\mu} \tilde{\pi}_{0}+\partial^{\mu} \tilde{\eta} \partial_{\mu} \tilde{\eta}+\partial^{\mu} \tilde{\eta}^{\prime} \partial_{\mu} \tilde{\eta}^{\prime}+\partial^{\mu} \tilde{K}_{L}^{0} \partial_{\mu} \tilde{K}_{L}^{0}+\partial^{\mu} \tilde{K}_{S}^{0} \partial_{\mu} \tilde{K}_{S}^{0}\right) \\
& -A_{W} \hat{g}_{8}\left(\partial^{\mu} \tilde{\pi}^{0} \partial_{\mu} \tilde{K}_{L}^{0}+\frac{1}{\sqrt{3}} \partial^{\mu} \tilde{\eta} \partial_{\mu} \tilde{K}_{L}^{0}\right)-A_{W} \tilde{g}_{8} \partial^{\mu} \tilde{\eta}^{\prime} \partial_{\mu} \tilde{K}_{L}^{0} \\
& +\frac{1}{2}\left(M_{\pi}^{2}\left(\tilde{\pi}^{0}\right)^{2}+M_{\eta}^{2} \tilde{\eta}^{2}+M_{\eta^{\prime}}^{2} \tilde{\eta}^{\prime 2}+M_{K}^{2}\left[\left(\tilde{K}_{L}^{0}\right)^{2}+\left(\tilde{K}_{S}^{0}\right)^{2}\right]\right)
\end{aligned}
$$

and the "physical fields" are given by

$$
\begin{aligned}
\tilde{\pi}^{0} & =\pi^{0}+\frac{M_{K}^{2}}{M_{K}^{2}-M_{\pi}^{2}} A_{W} \hat{g}_{8} K_{L}^{0} \\
\tilde{\eta} & =\eta-\frac{M_{K}^{2}}{M_{\eta}^{2}-M_{K}^{2}} \frac{A_{W} \hat{g}_{8}}{\sqrt{3}} K_{L}^{0} \\
\tilde{\eta}^{\prime} & =\eta^{\prime}-\frac{M_{K}^{2}}{M_{\eta^{\prime}}^{2}-M_{K}^{2}} A_{W} \tilde{g}_{8} K_{L}^{0} \\
\tilde{K}_{L}^{0} & =K_{L}^{0}-\frac{M_{\pi}^{2}}{M_{K}^{2}-M_{\pi}^{2}} A_{W} \hat{g}_{8} \pi^{0}+\frac{M_{\eta}^{2}}{M_{\eta}^{2}-M_{K}^{2}} \frac{A_{W} \hat{g}_{8}}{\sqrt{3}} \eta+\frac{M_{\eta^{\prime}}^{2}}{M_{\eta^{\prime}}^{2}-M_{K}^{2}} A_{W} \tilde{g}_{8} \eta^{\prime} \\
\tilde{K}_{S}^{0} & =K_{S}^{0}
\end{aligned}
$$

In terms of the physical fields the relevant contributions to $\mathcal{L}_{(2)}^{0}$ read

$$
\mathcal{L}_{(2)}^{0}=\frac{1}{2}\left(\partial^{\mu} \pi^{0} \partial_{\mu} \pi^{0}+\partial^{\mu} \eta \partial_{\mu} \eta+\partial^{\mu} \eta^{\prime} \partial_{\mu} \eta^{\prime}+\partial^{\mu} K_{S}^{0} \partial_{\mu} K_{S}^{0}\right.
$$




$$
\begin{gathered}
\left.+\left(1+A_{W}^{2} Q_{Z}\right) \partial^{\mu} K_{L}^{0} \partial_{\mu} K_{L}^{0}\right) \\
+\frac{1}{2}\left(M_{\pi}^{2}\left(\pi^{0}\right)^{2}+M_{\eta}^{2} \eta^{2}+M_{\eta^{\prime}}^{2} \eta^{\prime 2}+M_{K}^{2}\left(K_{S}^{0}\right)^{2}+M_{K}^{2}\left(1+A_{W}^{2} Q_{M}\right)\left(K_{L}^{0}\right)^{2}\right)
\end{gathered}
$$

where we note the difference in the kinetic and mass terms between $K_{S}^{0}$ and $K_{L}^{0}$, with

$$
\begin{array}{r}
Q_{Z}=-\frac{M_{K}^{2}\left(M_{K}^{2}-2 M_{\pi}^{2}\right) \hat{g}_{8}^{2}}{\left(M_{K}^{2}-M_{\pi}^{2}\right)^{2}}+\frac{1}{3} \frac{M_{K}^{2}\left(2 M_{\eta}^{2}-M_{K}^{2}\right) \hat{g}_{8}^{2}}{\left(M_{\eta}^{2}-M_{K}^{2}\right)^{2}}+\frac{M_{K}^{2}\left(2 M_{\eta^{\prime}}^{2}-M_{K}^{2}\right) \tilde{g}_{8}^{2}}{\left(M_{\eta^{\prime}}^{2}-M_{K}^{2}\right)^{2}} \\
Q_{M}=\frac{M_{K}^{2} M_{\pi}^{2} \hat{g}_{8}^{2}}{\left(M_{K}^{2}-M_{\pi}^{2}\right)^{2}}+\frac{1}{3} \frac{M_{K}^{2} M_{\eta}^{2} \hat{g}_{8}^{2}}{\left(M_{\eta}^{2}-M_{K}^{2}\right)^{2}}+\frac{M_{K}^{2} M_{\eta^{\prime}}^{2} \tilde{g}_{8}^{2}}{\left(M_{\eta^{\prime}}^{2}-M_{K}^{2}\right)^{2}}
\end{array}
$$

In consequence, the renormalized masses for $K_{L}^{0}, K_{S}^{0}$ read without the 1PI-irreducible contribution (C.3)

$$
M_{K_{L}}^{2}=M_{K}^{2}\left(\frac{1+A_{W}^{2} Q_{M}}{1+A_{W}^{2} Q_{Z}}\right), M_{K_{S}}^{2}=M_{K}^{2}
$$

This corresponds to a contribution of the mixing effect to the mass difference

$$
\begin{aligned}
\Delta\left(M_{K_{L}}-M_{K_{S}}\right) & =\frac{A_{W}^{2} M_{K}}{2}\left(Q_{M}-Q_{Z}\right) \\
& =\frac{A_{W}^{2} M_{K}}{2}\left(\frac{M_{K}^{2} \hat{g}_{8}^{2}}{M_{K}^{2}-M_{\pi}^{2}}-\frac{1}{3} \frac{M_{K}^{2} \hat{g}_{8}^{2}}{M_{\eta}^{2}-M_{K}^{2}}-\frac{M_{K}^{2} \tilde{g}_{8}^{2}}{M_{\eta^{\prime}}^{2}-M_{K}^{2}}\right) \\
& =-\frac{A_{W}^{2} M_{K}}{2}\left(0.465 \hat{g}_{8}^{2}+\tilde{g}_{8}^{2}\right)
\end{aligned}
$$

Summing (C.4) and (C.13) we obtain

$$
\frac{M_{K_{L}}-M_{K_{S}}}{\left(M_{K_{L}}-M_{K_{S}}\right)_{o b s}}=0.17\left(\frac{\mu_{W 2}^{2}}{f^{2}}-0.31 \hat{g}_{8}^{2}-\frac{2}{3} \tilde{g}_{8}^{2}\right)
$$

With typical values $\hat{g}_{8} \approx 1.5$ (cf. eq. (10.28)), and $\tilde{g}_{8} \approx 1$ this would imply $\mu_{W 2} \approx 300 \mathrm{MeV}$. This is of the order of the expected characteristic mass scale.

We complete this appendix by a discussion of the effective mixing between $K^{ \pm}$and $\pi^{ \pm}$. The relevant contribution of $\tilde{W}$-exchange to the pseudoscalar kinetic term is given by the square of the term linear in $\partial_{\mu} \Pi$ in eq. (10.29)

$$
\begin{aligned}
\Delta \mathcal{L}_{\text {kin }}^{(W)} & =-M_{W}^{2}\left(W_{\mu}^{+}\right)^{(1)}\left(W^{-\mu}\right)^{(1)} \\
& =-\frac{g_{W}^{2} f^{2}}{2 M_{W}^{2}} \operatorname{Tr}\left\{\partial^{\mu} \Pi \hat{\lambda}_{+}^{\dagger}\right\} \operatorname{Tr}\left\{\partial_{\mu} \Pi \hat{\lambda}_{+}\right\} \\
& =-\frac{g_{W}^{2} f^{2}}{4 M_{W}^{2}} \partial^{\mu}\left(c_{\theta} \tilde{\pi}^{+}+s_{\theta} \tilde{K}^{+}\right) \partial_{\mu}\left(c_{\theta} \tilde{\pi}^{-}+s_{\theta} \tilde{K}^{-}\right)
\end{aligned}
$$


This has to be combined with similar terms in eq. (C.6). Here we use again $\tilde{K}^{ \pm}, \tilde{\pi}^{ \pm}$for the fields appearing in eq. (5.27) which correspond to the basis where the quark mass term (2.9) is diagonal. In presence of weak interactions the fields $\tilde{K}^{ \pm}, \tilde{\pi}^{ \pm}$have to be related to the fields $K^{ \pm}, \pi^{ \pm}$for the

physical pseudoscalar mesons. The latter have diagonal propagators. If we only consider effects linear in $G_{F}$, the relevant bilinear

$$
\begin{aligned}
\mathcal{L}_{(2)}^{K \pi}= & \partial^{\mu} \tilde{\pi}^{+} \partial_{\mu} \tilde{\pi}^{-}+\partial^{\mu} \tilde{K}^{+} \partial_{\mu} \tilde{K}^{-}+M_{\pi}^{2} \tilde{\pi}^{+} \tilde{\pi}^{-}+M_{K}^{2} \tilde{K}^{+} \tilde{K}^{-} \\
& +A_{W}\left(\hat{g}_{8}-1\right)\left(\partial^{\mu} \tilde{K}^{+} \partial_{\mu} \tilde{\pi}^{-}+\partial^{\mu} \tilde{\pi}^{+} \partial_{\mu} \tilde{K}^{-}\right)
\end{aligned}
$$

is diagonalized by

$\tilde{\pi}^{+}=\pi^{+}-\frac{M_{K^{2}}}{M_{K^{2}}-M_{\pi^{2}}} A_{W}\left(\hat{g}_{8}-1\right) K^{+}, \tilde{K}^{+}=K^{+}+\frac{M_{\pi^{2}}}{M_{K^{2}}-M_{\pi^{2}}} A_{W}\left(\hat{g}_{8}-1\right) \pi^{+}$

such that

$$
\mathcal{L}_{(2)}^{K \pi}=\partial^{\mu} \pi^{+} \partial_{\mu} \pi^{-}+\partial^{\mu} K^{+} \partial_{\mu} K^{-}+M_{\pi}^{2} \pi^{+} \pi^{-}+M_{K}^{2} K^{+} K^{-}
$$

This mixing effect is not relevant for the hadronic kaon two-body decays since there are no cubic vertices involving only pseudoscalars in the absence of weak interactions. It contributes, however, to the decay of $K_{L}^{0}$ into three pions. Furthermore, it leads to an additional contribution to the strangenessviolating vertices between baryons and pseudoscalars.

\section{References}

[1] C. Wetterich, Phys. Lett. B462 (1999) 164

[2] C. Wetterich, hep-ph/9908514

[3] T. Banks, E. Rabinovici, Nucl. Phys. B160 (1979) 349;

E. Fradkin, S. Shenker, Phys. Rev. D19 (1979) 3682

[4] M. Reuter, C. Wetterich, Nucl. Phys. B408 (1993) 91;

C. Wetterich, "Electroweak Physics and the Early Universe", eds. J. Romao and F. Freire, Plenum Press (1994) 229;

W. Buchmüller, O. Philipsen, Nucl. Phys. B443 (1995) 47;

K. Kajantie, M. Laine, R. Rummukainen, M. Shaposhnikov, Phys. Rev. Lett. 77 (1996) 2887

[5] P. Damgaard, U. Heller, Phys. Lett. 171 B (1986), 442; Nucl. Phys. B294 (1987) 253; B304 (1988) 63;

H. Evertz, J. Jersak, K. Kanaya, Nucl. Phys. B285 (1987) 229 
[6] G. 't Hooft, in: Recent Developments in Gauge Theories (Plenum, New York, 1980), p. 135;

S. Dimopoulos, S. Raby, L. Susskind, Nucl. Phys. B173 (1980) 208;

T. Matsumoto, Phys. Lett. 97B (1980) 131;

M. Yasuè, Phys. Rev. D42 (1990) 3169

[7] K. Bardakci, M. B. Halpern, Phys. Rev. D6 (1972) 696

[8] C. Wetterich, Z. Phys. C57 (1993) 451

[9] D. Bailin, A. Love, Phys. Rep. 107 (1984) 325;

M. Alford, K. Rajagopal, F. Wilczek, Phys. Lett. 422B (1998) 247; Nucl. Phys. B537 (1999) 443;

R. Rapp, T. Schäfer, E. Shuryak, M. Velkovsky, Phys. Rev. Lett. 81 (1998) 53; J. Berges, K. Rajagopal, Nucl. Phys. B538 (1999) 214

[10] T. Schäfer, F. Wilczek, Phys. Rev. Lett. 82 (1999) 3956;

M. Alford, J. Berges, K. Rajagopal, Nucl. Phys. B558 (1999) 219

[11] C. Wetterich, Z. Phys. C48 (1990) 693

[12] G. 't Hooft, Phys. Rev. D14 (1976) 3432;

M. Shifman, A. Vainshtein, V. Zakharov, Nucl. Phys. B163 (1980) 46;

M. Nowak, J. Verbaarschot, I. Zahed, Nucl. Phys. B324 (1989) 1;

T. Schäfer, E. Shuryak, Rev. Mod. Phys. 70 (1998) 323

[13] D. Jungnickel, C. Wetterich, Phys. Rev. D53 (1996) 5142;

J. Berges, D. Jungnickel, C. Wetterich, Phys. Rev. D59 (1999) 034010

[14] C. Wetterich, Phys. Lett. 301B (1993) 90; Nucl. Phys. B352 (1991) 529; J. Berges, N. Tetradis, C. Wetterich, hep-ph/0005122

[15] D. Jungnickel, C. Wetterich, Eur. Phys. J. C1 (1998) 669

[16] D. Jungnickel, C. Wetterich, Phys. Lett. B389 (1996) 600

[17] M. Gell-Mann, M. Levy, Nuovo Cim. 16 (1960) 705;

A. Manohar, H. Georgi, Nucl. Phys. B234 (1984) 189

[18] J. Berges, D. Jungnickel, C. Wetterich, hep-ph/9811387

[19] M. Bando, T. Kugo, K. Yamawaki, Phys. Rep. 164 (1988) 217

[20] K. Kawarabayahi, M. Suzuki, Phys. Rev. Lett 16 (1966) 255;

Riazuddin and Fayyazuddin, Phys. Rev. 147 (1961) 1071

[21] M.-A. Halasz, J. Steele, G. Li, G. Brown, nucl-th/9712006

[22] B. Serot, D. Walecka, Int. J. Mod. Phys. E6 (1997) 515 
[23] J. Gasser, H. Leutwyler, Phys. Rep. C87 (1982) 77; Nucl. Phys. B250 (1985) 465

[24] J. Bijnens, J. G. Ecker, J. Gasser, DAPHNE physics handbook, hep$\mathrm{ph} / 9411232$

[25] D. Jungnickel, C. Wetterich, Eur. Phys. J. C2 (1998) 557

[26] J. G. Ecker, J. Gasser, A. Pich, E. de Rafael, Nucl. Phys. B321 (1989) 311 